University of Nebraska - Lincoln DigitalCommons@University of Nebraska - Lincoln

USGS Staff -- Published Research

US Geological Survey

2014

\title{
Real-time inversions for finite fault slip models and rupture geometry based on high-rate GPS data
}

\author{
S. E. Minson \\ USGS Earthquake Science Center, Seattle, Washington, California Institute of Technology \\ Jessica Murray \\ USGS Earthquake Science Center \\ John Langbein \\ USGS Earthquake Science Center \\ Joan Gomberg \\ USGS Earthquake Science Center, Seattle, Washington
}

Follow this and additional works at: http://digitalcommons.unl.edu/usgsstaffpub

Minson, S. E.; Murray, Jessica; Langbein, John; and Gomberg, Joan, "Real-time inversions for finite fault slip models and rupture geometry based on high-rate GPS data" (2014). USGS Staff -- Published Research. 819.

http://digitalcommons.unl.edu/usgsstaffpub/819

This Article is brought to you for free and open access by the US Geological Survey at DigitalCommons@University of Nebraska - Lincoln. It has been accepted for inclusion in USGS Staff -- Published Research by an authorized administrator of DigitalCommons@University of Nebraska - Lincoln. 


\section{Journal of Geophysical Research: Solid Earth}

\author{
RESEARCH ARTICLE \\ 10.1002/2013JB010622

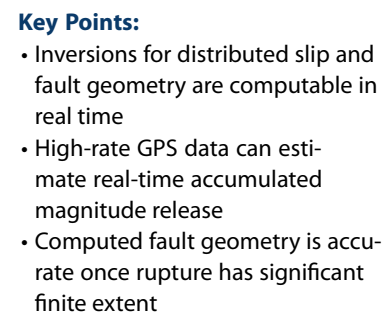

- Inversions for distributed slip and fault geometry are computable in real time

- High-rate GPS data can estimate real-time accumulated magnitude release

- Computed fault geometry is accurate once rupture has significant finite extent

Supporting Information:

- Readme

- Figures S1-S15

- Movies S1-S9

Correspondence to:

S. E. Minson,

minson@gps.caltech.edu

Citation:

Minson, S. E., J. R. Murray, J. O. Langbein, and J. S. Gomberg (2014), Real-time inversions for finite fault slip models and rupture geometry based on high-rate GPS data, J. Geophys. Res. Solid Earth, 119, 3201-3231, doi:10.1002/2013JB010622.

Received 22 AUG 2013 Accepted 23 FEB 2014 Accepted article online 27 FEB 2014 Published online 17 APR 2014

\section{Real-time inversions for finite fault slip models and rupture geometry based on high-rate GPS data}

\author{
S. E. Minson ${ }^{1,2}$, Jessica R. Murray ${ }^{3}$, John O. Langbein ${ }^{3}$, and Joan S. Gomberg ${ }^{1}$ \\ ${ }^{1}$ USGS Earthquake Science Center, Seattle, Washington, USA, ${ }^{2}$ Now at Seismological Laboratory, Division of Geological \\ and Planetary Sciences, California Institute of Technology, Pasadena, California, USA, ${ }^{3}$ USGS Earthquake Science Center, \\ Menlo Park, California, USA
}

Abstract We present an inversion strategy capable of using real-time high-rate GPS data to simultaneously solve for a distributed slip model and fault geometry in real time as a rupture unfolds. We employ Bayesian inference to find the optimal fault geometry and the distribution of possible slip models for that geometry using a simple analytical solution. By adopting an analytical Bayesian approach, we can solve this complex inversion problem (including calculating the uncertainties on our results) in real time. Furthermore, since the joint inversion for distributed slip and fault geometry can be computed in real time, the time required to obtain a source model of the earthquake does not depend on the computational cost. Instead, the time required is controlled by the duration of the rupture and the time required for information to propagate from the source to the receivers. We apply our modeling approach, called Bayesian Evidence-based Fault Orientation and Real-time Earthquake Slip, to the 2011 Tohoku-oki earthquake, 2003 Tokachi-oki earthquake, and a simulated Hayward fault earthquake. In all three cases, the inversion recovers the magnitude, spatial distribution of slip, and fault geometry in real time. Since our inversion relies on static offsets estimated from real-time high-rate GPS data, we also present performance tests of various approaches to estimating quasi-static offsets in real time. We find that the raw high-rate time series are the best data to use for determining the moment magnitude of the event, but slightly smoothing the raw time series helps stabilize the inversion for fault geometry.

\section{Introduction}

Earthquake early warning (EEW) algorithms aim to estimate the location and magnitude of an earthquake in real time and use that information to both issue warnings and as input into shaking simulations which can then be used to issue more informative warnings. However, there are many additional source parameters which, if known in real time, could improve the usefulness of EEW significantly. For example, real-time information about the faulting mechanism or the spatial distribution of slip could improve shaking forecasts and tsunami hazard assessments. (The fault geometry of the source is particularly important for evaluating tsunami hazard from an offshore event since a transform event is less likely to produce a tsunami than a thrust event of the same magnitude.) Knowing the locations of large asperities or even simply the spatial extent of rupture could help in determining where emergency response resources should be deployed.

All of the above source parameters could be known if we could solve for a distributed earthquake source model in real time as the earthquake rupture propagates. Performing a finite fault slip inversion can be computationally expensive even though it is fundamentally a linear inverse problem (for linear elastic constitutive laws). Moreover, finite fault slip models are traditionally calculated by utilizing a known, fixed fault geometry. However, in an EEW setting, we do not know the rupture geometry a priori. Thus, it will be necessary to simultaneously solve for the fault geometry, which is a nonlinear inverse problem. Here we introduce an approach that combines an analytical solution to the linear finite fault slip inversion problem with a parallelizable exploration of potential rupture geometries. The algorithm produces a solution to the simultaneous inversion for slip and rupture geometry that is so computationally efficient it can be computed and updated in real time. Since we use Bayesian inference to obtain our solutions, our approach yields not only real-time finite fault slip models but also uncertainties on our model parameters. 


\section{Semianalytical Bayesian Finite Fault Inversions}

Let us assume that we have real-time observations of quasi-static surface offsets as they accumulate during the earthquake rupture. (Our suggested approach to estimating real-time static offsets from high-rate GPS time series is discussed in section 3.) In traditional, post-earthquake finite fault slip inversions, the distributed slip model is usually parameterized by discretizing a fault of known size, orientation, and location. In a real-time setting, we can expect to know the earthquake's epicenter from the existing EEW infrastructure, although we may not know the focal depth since most current EEW systems do not estimate the hypocentral depth. (This approach is practicable only in regions with sufficient instruments, telemetry, and processing infrastructure for both seismic and real-time high-rate GPS-based EEW.) However, we cannot assume that we will have any information about the rupture size or fault orientation. The size of the rupture obviously cannot be known a priori because in real time the earthquake is evolving and growing in size. Because a distributed slip inversion solves for slip at each point on a model fault plane, it is not necessary that the fault plane be the same size as the actual rupture. As long as our fault plane is at least as large as the eventual size of the rupture we are modeling, the inversion should assign zero slip to the excess fault area and, because we adopt an analytical solution to the slip inversion, the additional computational cost of including extra subfaults is not prohibitive.

Given location information from existing EEW methods and using a priori knowledge of the anticipated largest rupture, we then have to solve in real time for the fault orientation and the distribution of slip on the fault. By knowing a location that the fault plane intersects (e.g., the earthquake hypocenter), the geometry of the fault plane can be described with just two parameters: the strike and dip of the fault plane. For any fault geometry, solving for slip at various points on the fault plane is a linear inverse problem. In practice, however, it is an underdetermined inverse problem with potentially many solutions. We avoid the instability associated with solutions to such problems and capture the nonuniqueness by using Bayesian analysis to infer both the ensemble of all plausible slip models for a particular fault geometry and the relative likelihood of each fault geometry. Further, we employ a Bayesian linear regression that has a closed-form solution so that the inversion for slip on a fault plane can be solved analytically. This leaves only two nonlinear parameters unknown (strike and dip). Because almost all model parameters can be determined analytically, the entire joint inversion for slip and fault geometry is calculable in real time.

Bayes' theorem [Bayes, 1763] gives the relationship between inverse conditional probabilities. Thus, for random variables $A$ and $B$, if $\mathrm{p}(A \mid B)$ is the conditional probability of $A$ given $B$, then

$$
\mathrm{p}(B \mid A)=\frac{\mathrm{p}(A \mid B) \mathrm{p}(B)}{\mathrm{p}(A)}
$$

Thus, it follows that the solution to any inverse problem can be expressed according to Bayes' theorem as

$$
\mathrm{p}(\boldsymbol{\theta} \mid \mathcal{D})=\frac{\mathrm{p}(\mathcal{D} \mid \boldsymbol{\theta}) \mathrm{p}(\boldsymbol{\theta})}{\mathrm{p}(\mathcal{D} \mid \mathcal{M})}
$$

where $\boldsymbol{\theta}$ is a vector containing the value of each model parameter and $\mathcal{D}$ is a vector of observations. The data likelihood, $\mathrm{p}(\mathcal{D} \mid \boldsymbol{\theta})$, is a probability density function (PDF) describing how well the predictions from a given set of model parameter values, $\boldsymbol{\theta}$, fit the observed data. The prior PDF, $\mathrm{p}(\boldsymbol{\theta})$, describes our a priori knowledge of the relative plausibility of different parameter values. The scalar $\mathrm{p}(\mathcal{D} \mid \mathcal{M})$ is the marginal likelihood or evidence in favor of a particular model class, $\mathcal{M}$, where a model class is a group of models characterized by the same set of parameters. (For example, all linear functions parameterized by a slope and intercept could be said to comprise one model class, $\mathcal{M}$, and each model in that model class is defined by a two-element vector, $\boldsymbol{\theta}$, containing the intercept and slope. Thus, any value of $\boldsymbol{\theta}$ uniquely defines one realization of a linear model, while $\mathcal{M}$ represents the family of all linear functions.) The posterior $\operatorname{PDF} \mathrm{p}(\boldsymbol{\theta} \mid \mathcal{D})$, is traditionally considered the solution to the inverse problem. The posterior PDF is the probability of any potential set of model parameter values, $\boldsymbol{\theta}$, given our a priori constraints, $\mathrm{p}(\boldsymbol{\theta})$, and the misfit between the predictions of our forward model and the data, $\mathrm{p}(\mathcal{D} \mid \boldsymbol{\theta})$, normalized by the evidence in favor of the model class $(\mathcal{M}), \mathrm{p}(\mathcal{D} \mid \mathcal{M})$.

For our purposes, the "model class" refers to the particular fault geometry (strike and dip) assumed. In many applications, the inverse problem is solved by evaluating $\mathrm{p}(\boldsymbol{\theta} \mid \mathcal{D}) \propto \mathrm{p}(\mathcal{D} \mid \boldsymbol{\theta}) \mathrm{p}(\boldsymbol{\theta})$ and ignoring the constant of proportionality which is the marginal likelihood, $\mathrm{p}(\mathcal{D} \mid \mathcal{M})$. But in our case, $\mathrm{p}(\mathcal{D} \mid \mathcal{M})$ is an important quantity 
as it is the probability associated with our model design, i.e., our choice of fault geometry. Effectively, the marginal likelihood, $\mathrm{p}(\mathcal{D} \mid \mathcal{M})$, helps measure the probability of a particular fault geometry given all potential slip models inferred for that fault geometry. Equation (2) is a common shorthand notation. But the reason that $\mathrm{p}(\mathcal{D} \mid \mathcal{M})$ can be used to infer the optimal model class can be more clearly seen by rewriting equation (2) to explicitly include the model class, $\mathcal{M}$ :

$$
\mathrm{p}(\boldsymbol{\theta} \mid \mathcal{D}, \mathcal{M})=\frac{\mathrm{p}(\mathcal{D} \mid \boldsymbol{\theta}, \mathcal{M}) \mathrm{p}(\boldsymbol{\theta} \mid \mathcal{M})}{\mathrm{p}(\mathcal{D} \mid \mathcal{M})}
$$

To infer which model design is best based on the observed data, we want to find $p(\mathcal{M} \mid \mathcal{D})$. This quantity can be obtained by applying Bayes' theorem (equation (1)) to the denominator of equation (3):

$$
\mathrm{p}(\mathcal{M} \mid D) \propto \mathrm{p}(\mathcal{D} \mid \mathcal{M}) \mathrm{p}(\mathcal{M})
$$

where $p(\mathcal{M})$ represents our prior preferences for each model class. If we assume that all model classes are equally likely a priori, then maximizing $\mathrm{p}(\mathcal{D} \mid \mathcal{M})$ is equivalent to maximizing $\mathrm{p}(\mathcal{M} \mid \mathcal{D})$.

The marginal likelihood is a principled method for identifying which of a set of possible model classes is optimal: it favors models that fit the data better while penalizing those that are needlessly complex, where the complexity of the model is measured as deviation from the prior PDF. (See Appendix A for details.) By evaluating the marginal likelihood for all model classes (i.e., all fault geometries), we will find the relative probabilities of all fault geometries and thus which fault geometry is most likely. By evaluating the posterior PDF for our preferred fault geometry, we will obtain the ensemble of all plausible slip models on that fault plane. Furthermore, by employing the Bayesian model class selection framework, we are assured that the solution will adapt appropriately if we were to change our slip priors or the prior information on the fault geometry.

The key to solving for the fault geometry and slip distribution with sufficient speed for real-time applications is to use analytical solutions for almost all of the model parameters so that only a very few model parameters must be inferred by numerical methods. By using an analytical solution for our slip inversion and fixing the fault location, the only nonanalytical part of the inversion is solving for the fault orientation uniquely described by two parameters (strike and dip). In general, the number of samples required to describe the space of model parameters increases exponentially with the number of free parameters in what is called the "curse of dimensionality" [Bellman, 1957]. But since we have written the problem so that there are only two free parameters that cannot be determined analytically, it is computationally feasible to sweep through the limited domain of candidate fault geometries in real time. The domain of possible strikes and dips can be explored using a variety of methods, including a simple grid search or a variety of Monte Carlo sampling algorithms [Mosegaard and Tarantola, 1995] such as the Metropolis algorithm [Metropolis et al., 1953; Chib and Greenberg, 1995]. Because evaluating the slip inversion and marginal likelihood for a given fault geometry is independent of the other fault geometries, the search over strike and dip is embarrassingly parallel and thus well suited to computing in parallel across multiple processors.

We use conjugate priors to obtain closed-form expressions for the marginal likelihood (i.e., the evidence for a particular choice of fault geometry), $\mathrm{p}(\mathcal{D} \mid \mathcal{M})$, and the posterior PDF (i.e., the PDF describing the distribution of possible slip models on that fault plane and the regression variance), $p(\theta \mid D)$. A conjugate prior is any prior PDF such that the posterior PDF $\mathrm{p}(\boldsymbol{\theta} \mid \mathcal{D}) \propto \mathrm{p}(\mathcal{D} \mid \boldsymbol{\theta}) \mathrm{p}(\boldsymbol{\theta})$ belongs to the same family as the prior PDF, $\mathrm{p}(\boldsymbol{\theta})$ (i.e., the prior and posterior PDFs have the same functional form). We adopt the well-known normal-inverse-gamma conjugate prior for linear regression with unknown variance. This particular conjugate prior requires us to use a Gaussian prior PDF on our slip model, $\boldsymbol{\beta}$, and an inverse-gamma prior for updating the regression variance, $\sigma^{2}$. The details of the normal-inverse-gamma prior, proof of its conjugacy, and derivations of the marginal likelihood and posterior mean and covariance of our model parameters are given in Appendix B following O'Hagan [1994]. The relevant results from the appendix are

$$
\mathrm{p}(\mathcal{D} \mid \mathcal{M})=\frac{1}{(2 \pi)^{n / 2}|\mathbf{D}|^{1 / 2}} \frac{\left|\mathbf{V}^{*}\right|^{1 / 2}}{|\mathbf{V}|^{1 / 2}} \frac{(a / 2)^{d / 2}}{\left(a^{*} / 2\right)^{d^{*} / 2}} \frac{\Gamma\left(d^{*} / 2\right)}{\Gamma(d / 2)}
$$

with

$$
\mathbf{V}^{*}=\left(\mathbf{V}^{-1}+\mathbf{X}^{T} \mathbf{D}^{-1} \mathbf{X}\right)^{-1}
$$




\section{At each time step following the origin time:}

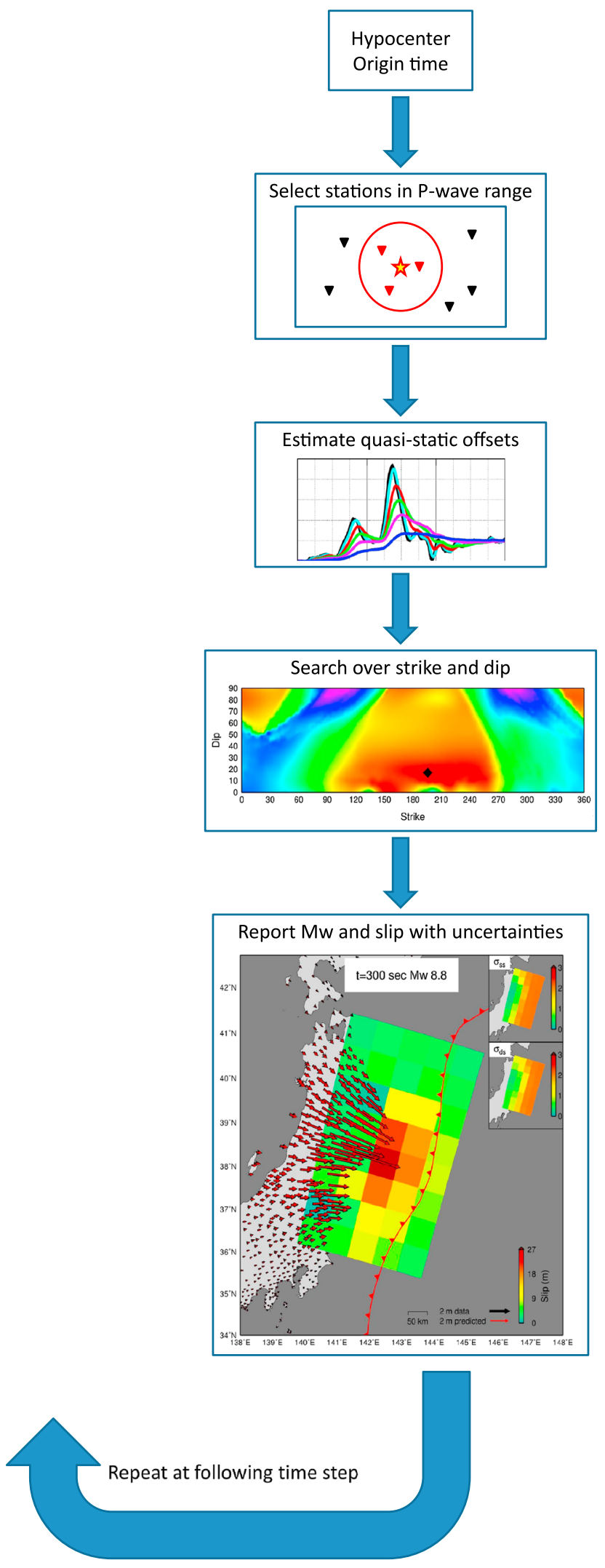

Figure 1 


$$
\begin{gathered}
\mathbf{m}^{*}=\left(\mathbf{V}^{-1}+\mathbf{X}^{T} \mathbf{D}^{-1} \mathbf{X}\right)^{-1}\left(\mathbf{V}^{-1} \mathbf{m}+\mathbf{X}^{T} \mathbf{D}^{-1} \mathbf{y}\right) \\
=\mathbf{V}^{*}\left(\mathbf{V}^{-1} \mathbf{m}+\mathbf{X}^{T} \mathbf{D}^{-1} \mathbf{y}\right) \\
a^{*}=a+\mathbf{m}^{T} \mathbf{V}^{-1} \mathbf{m}+\mathbf{y}^{T} \mathbf{D}^{-1} \mathbf{y}-\left(\mathbf{m}^{*}\right)^{T}\left(\mathbf{V}^{*}\right)^{-1} \mathbf{m}^{*} \\
d^{*}=d+n
\end{gathered}
$$

where $\mathbf{y}$ is an $n \times 1$ vector of observations, $\mathbf{X}$ is our $n \times p$ design matrix (matrix of Green's functions for a given fault orientation defined by strike, $\phi$, and dip, $\delta$ ), $\mathbf{D}$ is the $n \times n$ covariance matrix of errors, $\mathbf{m}$ (which is $p \times 1$ ) and $\mathbf{V}$ (which is $p \times p$ ) are the prior mean and variance on our slip model, respectively. Since we solve for strike-slip and dip-slip motions on each of our fault patches, $p$ will be twice the number of fault patches. Thus, $\boldsymbol{\theta}$ is a $(p+1) \times 1$ vector containing $\boldsymbol{\beta}$ (a vector composed of the two components of slip on each patch) and $\sigma^{2}$ (the regression variance), so that $\boldsymbol{\theta}=\left(\boldsymbol{\beta}, \sigma^{2}\right) . \Gamma(\cdot)$ denotes the gamma function. The prior PDF on the regression variance is defined by $a$ and $d$ which are, for our purposes, nuisance parameters that appear as part of the normal-inverse-gamma conjugate prior formulation of the inverse problem. Their influence decreases as they go to zero (equations (8) and (9)), and so we will always set them to some small arbitrary value.

Equation (5) is the marginal likelihood describing the relative plausibility of a particular model class. In our case, the model class is equivalent to the fault orientation. However, we are also interested in the posterior distribution of slip on each fault orientation, which can be obtained from equation (2) by integrating over all regression variances. It is shown in Appendix B that the resulting posterior PDF on slip is a multivariate $t$ distribution for $d^{*}$ degrees of freedom with mean, $\mathbf{m}^{*}$ (equation (7)), and covariance

$$
\frac{a^{*}}{d^{*}-2} \mathbf{V}^{*}
$$

where $\mathbf{V}^{*}, a^{*}$, and $d^{*}$ are given by equations (6), (8), and (9), respectively.

The optimal fault orientation can be found by maximizing $\mathrm{p}(\mathcal{M} \mid \mathcal{D})$ (equation (4)). However, in our implementation, we assume that we have no a priori knowledge of the fault orientation, i.e., our prior PDF on the fault orientation, $\mathrm{p}(\mathcal{M})$, is uniform. Thus, maximizing $\mathrm{p}(\mathcal{M} \mid \mathcal{D})$ is equivalent to maximizing the marginal likelihood, $\mathrm{p}(\mathcal{D} \mid \mathcal{M})$. (Alternatively, if we wanted our prior constraints on the fault orientation to know about historical seismicity in a given region, we could use $\mathrm{p}(\mathcal{M})$ proportional to the relative frequency of observed focal mechanism orientations and maximize $\mathrm{p}(\mathcal{D} \mid \mathcal{M}) \mathrm{p}(\mathcal{M})$.) Specifically, we assume that all orientations of the fault normal vector are equally likely. This is not equivalent to using uniform prior PDFs on strike, $\phi$, and $\operatorname{dip}, \delta$, because a uniform prior on dip would oversample shallowly dipping faults because the area element in spherical coordinates is a function of colatitude. Instead, to obtain a uniform distribution of fault normal vectors on a sphere, we use a uniform prior on strike $\left[\phi \sim V\left(0^{\circ}, 360^{\circ}\right)\right]$ while the prior PDF on dip is given by $u \sim \mathcal{V}(0,1)$, where $\delta=\cos ^{-1}(u)$. Thus, our uniform prior PDF is a uniform distribution on $\mathcal{M}=\mathcal{M}(\phi, \cos \delta)$.

Our Bayesian inversion methodology for fault geometry and distributed slip can be summarized as follows (Figure 1):

1. For each of a suite of potential fault orientations, evaluate the marginal likelihood, $\mathrm{p}(\mathcal{D} \mid \mathcal{M})$ (equation (5)), associated with Bayesian linear regression of a slip model on that fault plane.

2. Select the strike and dip that maximizes $\mathrm{p}(\mathcal{D} \mid \mathcal{M})$ as the most likely fault geometry.

3. For the most likely fault geometry, report the posterior slip model associated with that fault plane. Useful summary statistics of the posterior include the mean posterior slip model, $\mathbf{m}^{*}$ (equation (7)), the standard deviation associated with each slip (the square root of the diagonal elements of the posterior covariance matrix in equation (10)), and the moment magnitude calculated from the mean posterior slip model.

We will refer to this inversion methodology as Bayesian Evidence-based Fault Orientation and Real-time Earthquake Slip (BEFORES).

Figure 1. Flowchart showing progression of simultaneous inversion for slip and fault geometry as a function of time. The algorithm is triggered by an event hypocenter (or epicenter) and origin time. For each time after the trigger, stations within $P$ wave range of the epicenter are chosen for inclusion in the inversion and quasi-static offsets are estimated for those stations. The next step is to evaluate the marginal likelihood (or evidence) for all fault geometries. Rupture parameters are reported for the slip model associated with the fault geometry that maximizes the marginal likelihood. 

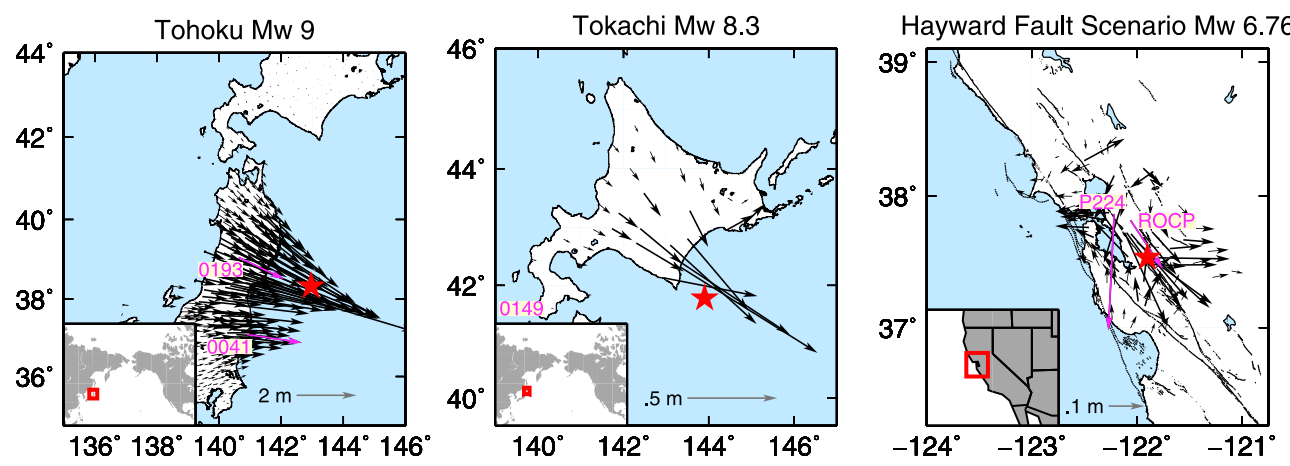

Figure 2. Location maps of real and scenario earthquakes used in performance tests. The red stars mark the assumed hypocenter locations. Black vectors are the horizontal components of the final estimated static offsets. Displacement time series for stations marked in pink are shown in Figure 3.

\section{Data}

In section 2, we designed a static slip inversion that is driven by a set of quasi-static surface displacements estimated at each second (or whatever interval is desired for updating the model) during the earthquake rupture. This is now operationally possible due to the introduction of real-time high-rate GPS networks. In theory, surface displacements could be derived from seismic data, but broadband velocity seismograms can clip for large earthquakes, and double integration of acceleration records can yield unstable and inaccurate estimates of displacements. In contrast, real-time high-rate GPS data do not suffer from clipping and directly measure surface displacements in real time.
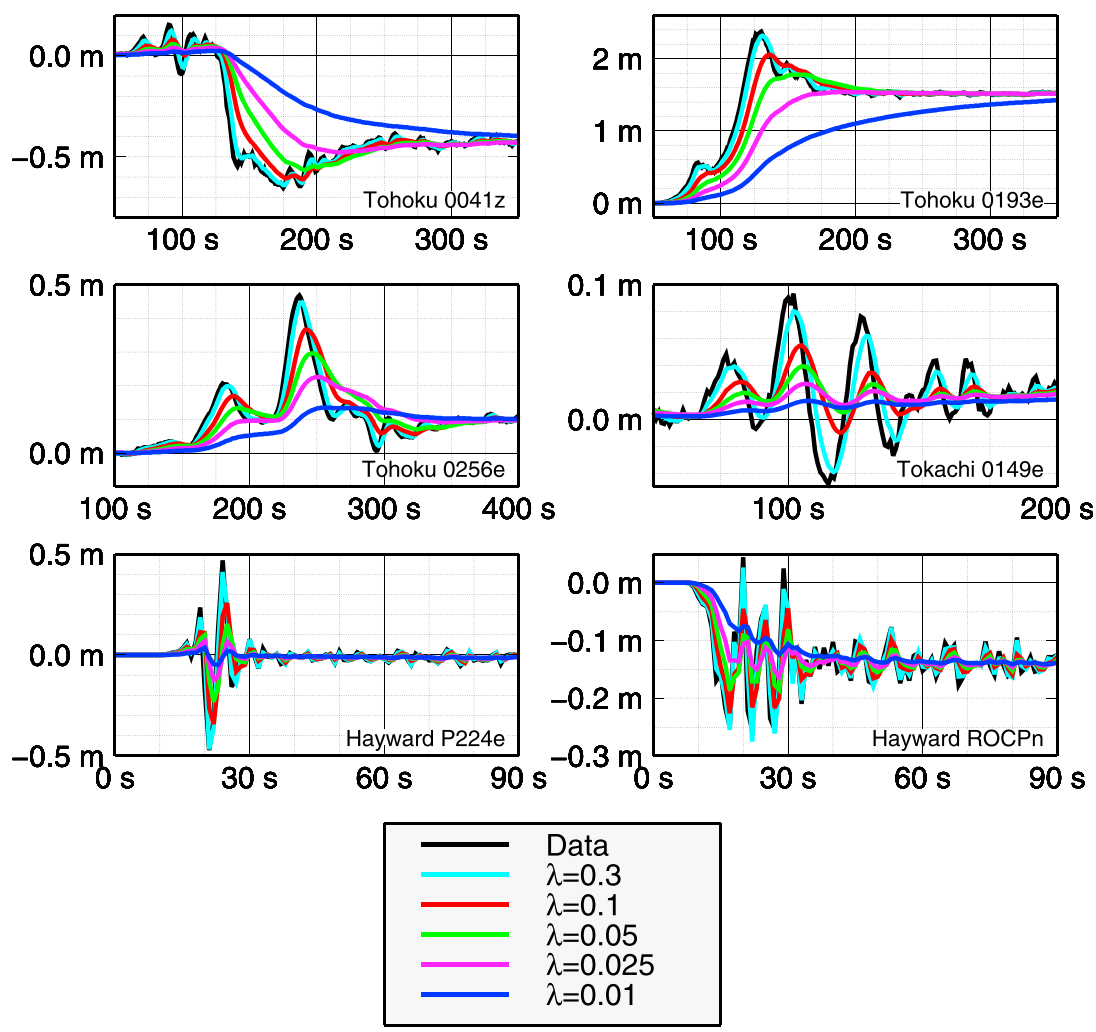

Figure 3. Comparison of smoothed displacement time series for selected stations in Figure 2. The original observed (or simulated in the case of the Hayward fault scenario) displacement time series are shown in black. The colored traces are these same time series smoothed using an EWMA for various values of the decay constant, $\lambda$. 


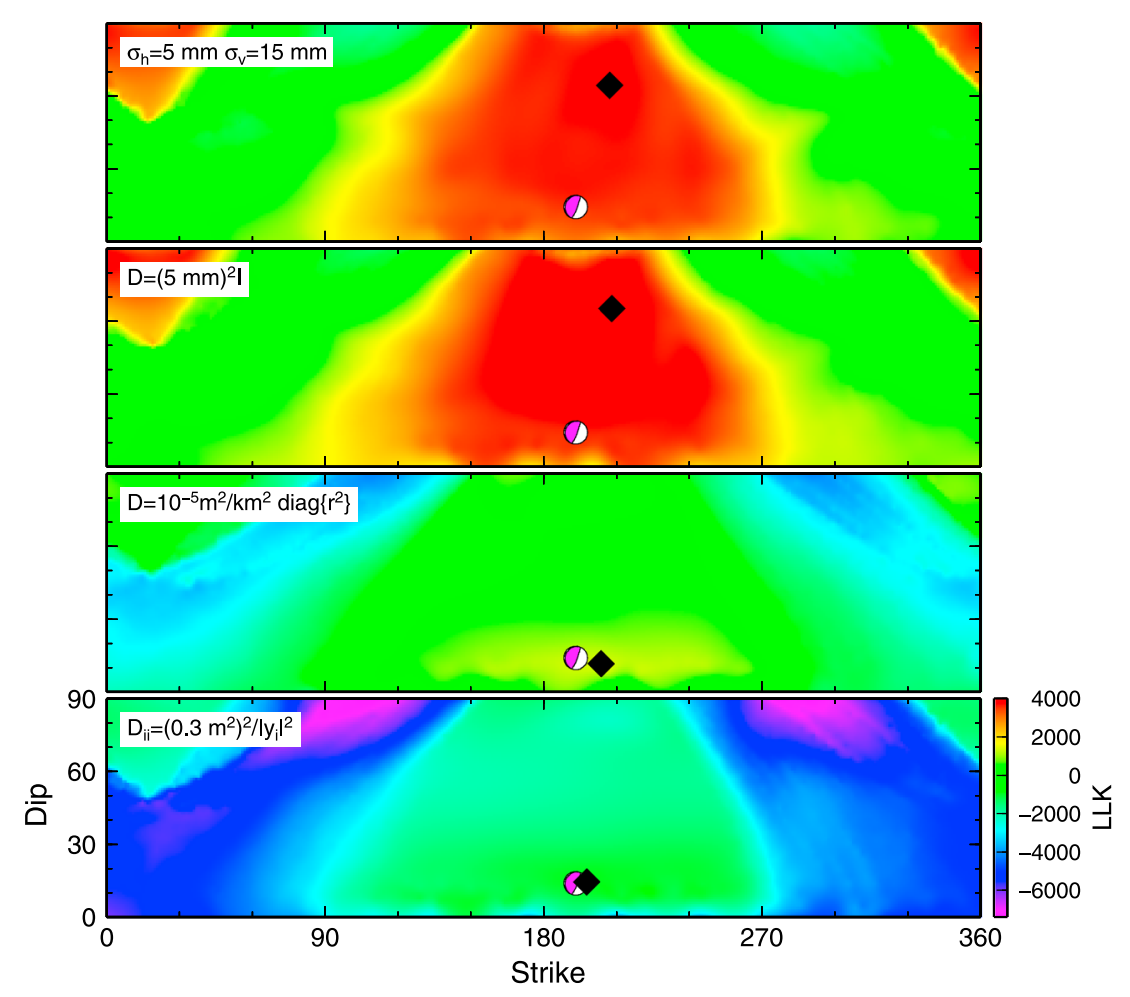

Figure 4. Results of geometry inversion at $300 \mathrm{~s}$ after the origin time of the 2011 Tohoku-oki earthquake using different data covariance matrices. The log of the marginal likelihood (LLK) is plotted for all values of strike and dip. The most likely fault geometry found by inversion is marked with a black diamond. The geometry determined from a W phase focal mechanism is shown with a pink focal mechanism [Duputel et al., 2011].

High-rate GPS data, like seismic data, record the dynamic wavefield as it propagates outward from the earthquake source. Our goal is to estimate the quasi-static component of these waveforms, i.e., the accumulation of permanent static offset as a function of time without any transitory contributions from body waves or surface waves. But it is not obvious how this subset of the full time-dependent displacement history can be obtained.

There have been several publications on the topic of deriving static offsets from high-rate GPS data. Allen and Ziv [2011] suggested using the short-term average versus long-term average (STA/LTA) triggering method of Allen [1978] to detect when the seismic waves reach a station, wait for one full oscillation of the waveform or some critical amount of time (whichever comes first) and then begin calculating a cumulative average of the displacement time series from that point in time. Similarly, Ohta et al. [2012] used a variation of the STA/LTA algorithm to detect the arrival of motion and, once triggered, began computing the difference between a $20 \mathrm{~s}$ moving average and a reference position for the station calculated 5 min previous to the trigger time. An even simpler approach was adopted by Crowell et al. [2012] and Melgar et al. [2012], who used moving averages of $50 \mathrm{~s}$ and $120 \mathrm{~s}$, respectively.

All of the above estimates of static offsets are derived from smoothed averages (either moving or cumulative averages) of the observed time series, although the different authors employed very different amounts of smoothing resulting in very different estimates of displacement as the rupture occurred. (In the long run, all of these methods will converge to the final static offset, although they converge at different rates and may produce differing offset estimates prior to convergence.) We can view this ensemble of data smoothing approaches as low-pass filtering.

To explore the effects of different smoothing choices, we employ an exponentially weighted moving average (EWMA) [Roberts, 1959], which is essentially a simple resistor-capacitor low-pass filter. An EWMA can be defined by

$$
\mathrm{EWMA}_{t}=\lambda z_{t}+(1-\lambda) \mathrm{EWMA}_{t-1}
$$



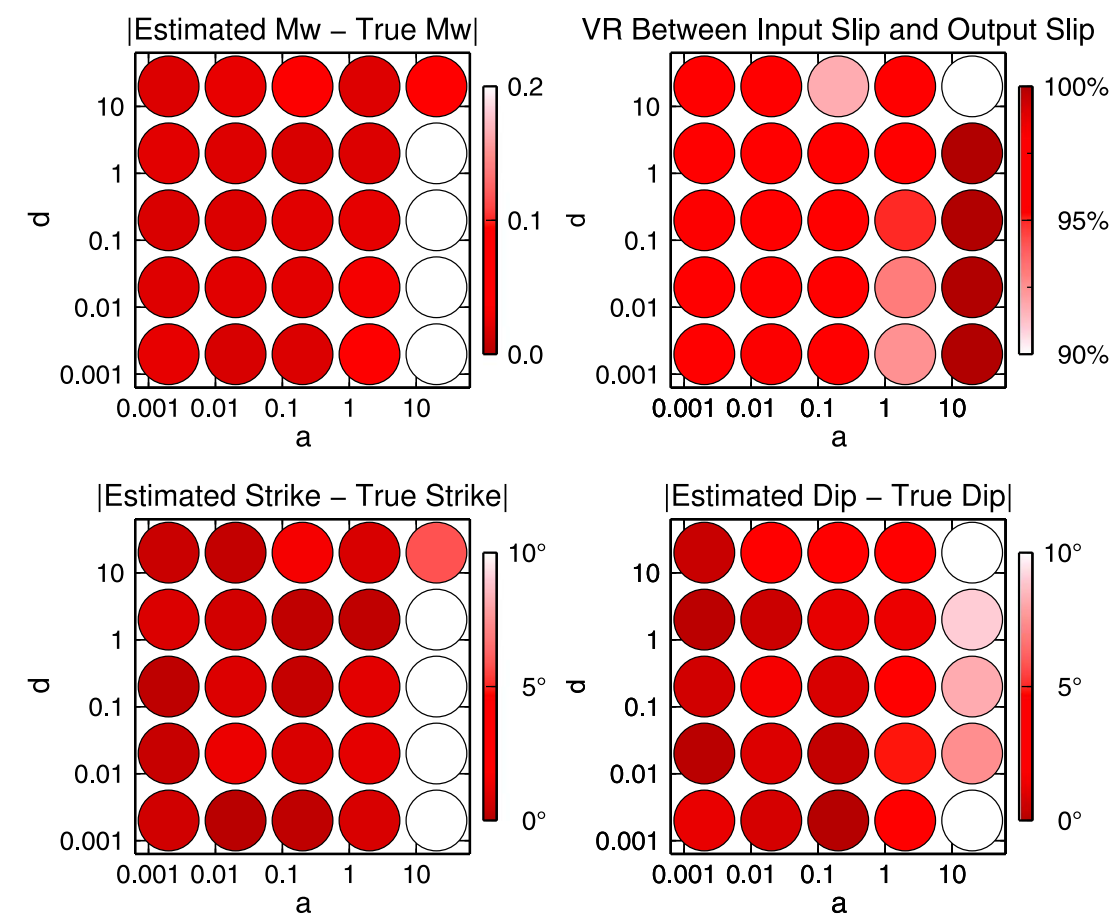

Figure 5. Effects of varying $a$ and $d$ on inversion results for Hayward fault simulation. The differences between the output moment magnitude, slip model, fault strike, fault dip, and the input values for each of these quantities are shown. The differences between the input and output slip vectors are quantified by their variance reduction, $\mathrm{VR}=100 \% \times\left[1-\frac{\Sigma_{i=1}^{p}\left(\mathbf{m}_{\left.\text {true }_{i}-\mathbf{m}_{i}{ }_{i}\right)^{2}}\right.}{\Sigma_{i=1}^{p} \mathbf{m}_{\text {true }_{i}}{ }_{i}}\right]$.

where $\lambda$ is a decay constant which controls the depth of memory of the EWMA $(0<\lambda \leq 1)$ and $z_{t}$ is the $t$ th sample of time series, $\mathbf{z}$.

For our performance tests we focused on three events, the 2011 great $M_{w} 9.0$ Tohoku-oki earthquake, the $2003 M_{w} 8.3$ Tokachi-oki earthquake, and a simulated $M_{w} 6.76$ earthquake on California's Hayward fault (Figure 2). (This simulation is scenario hs_r02_hypoF_vr92_tr15 in Aagaard et al. [2010].) The exponentially weighted moving average (using a variety of decay rates) of data from select stations for these earthquakes is shown in Figure 3 . As the decay rate, $\lambda$, decreases, the smoothed version of the data moves from something which is almost identical to the raw time series to something which is entirely devoid of any kinematic component. However, the highly smoothed time series also take substantially longer to reach the final permanent static offset than the original data do. We find that applying large amounts of smoothing adds a substantial and undesirable lag between the estimated magnitude and the actual magnitude, and can even introduce a lag into estimating the slip distribution and geometry of the rupture. (See section 5.)

\section{Sensitivity to Inversion Design}

Any inversion is highly dependent on the assumed errors in the observations and the assumed errors in the predictions of the forward model. The combined effects of these errors can be expressed as

$$
\mathbf{D}=\mathbf{C}_{\mathrm{d}}+\mathbf{C}_{\mathrm{p}}
$$

where $\mathbf{C}_{\mathbf{d}}$ and $\mathbf{C}_{\mathbf{p}}$ are covariance matrices for the observational errors and the prediction errors, respectively. (For a more complete introduction to the role of observational and prediction errors in finite fault source modeling, see, e.g., Minson et al. [2013].) These errors should ideally be updated as part of the inversion process, but this is not always computationally feasible. For our inversions, we experimented with a number of different forms for the covariance matrix $\mathbf{D}$ (Figure 4). We found setting $\mathbf{D}$ to the formal observational errors of $5 \mathrm{~mm}$ and $15 \mathrm{~mm}$ for the horizontal and vertical components of the surface offsets, respectively, did a poor job of recovering the strike and dip of the fault plane (Figure 4, first row). This is not due to the effects of down weighting the contribution from the vertical observations because using an uncertainty of $5 \mathrm{~mm}$ 

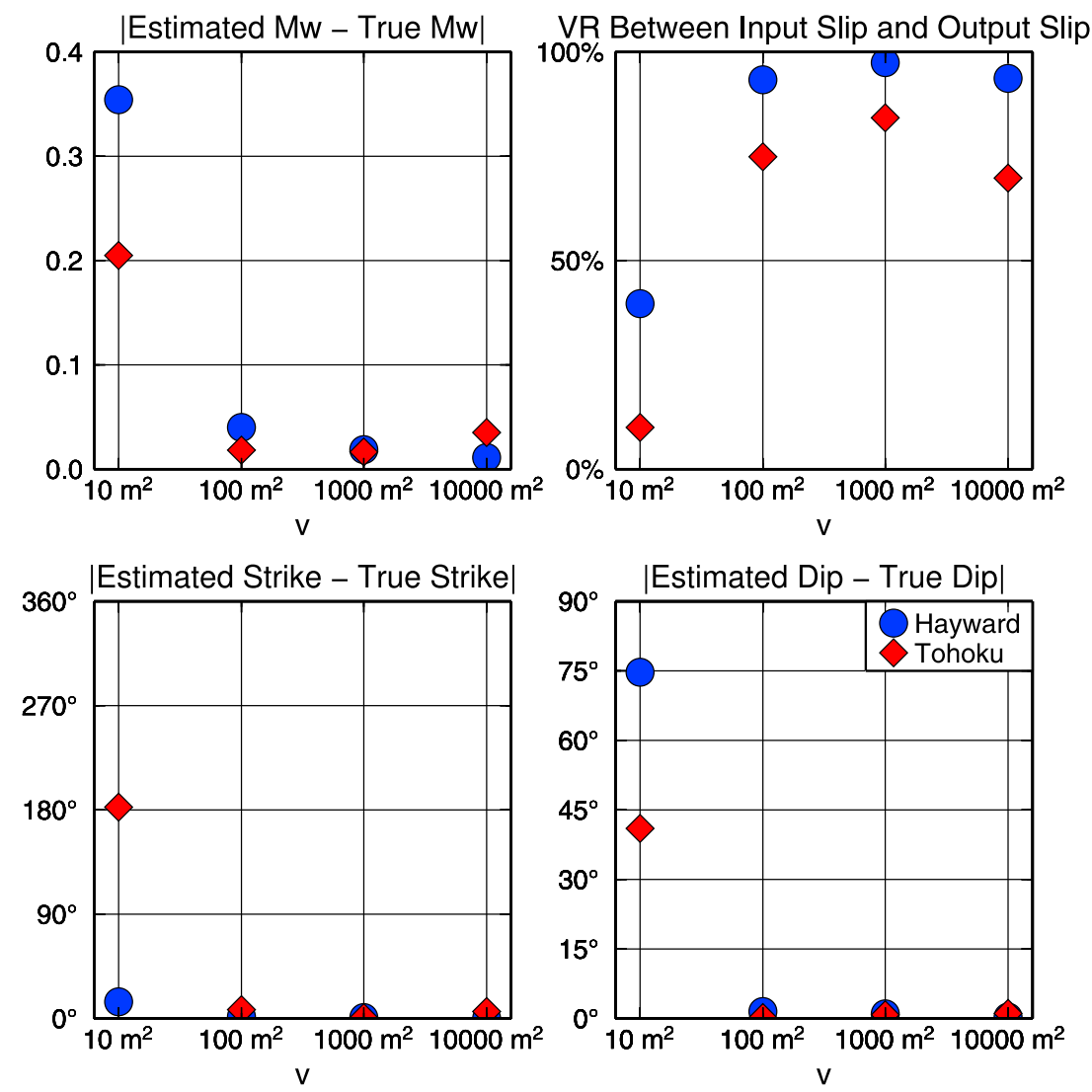

Figure 6. Effects on inversion results of varying $v$. The differences between the output moment magnitude, slip model, fault strike, fault dip, and the input values for each of these quantities are shown for both an $M_{w} 7$ Hayward fault simulation and $M_{w} 9$ Tohoku-oki simulation.
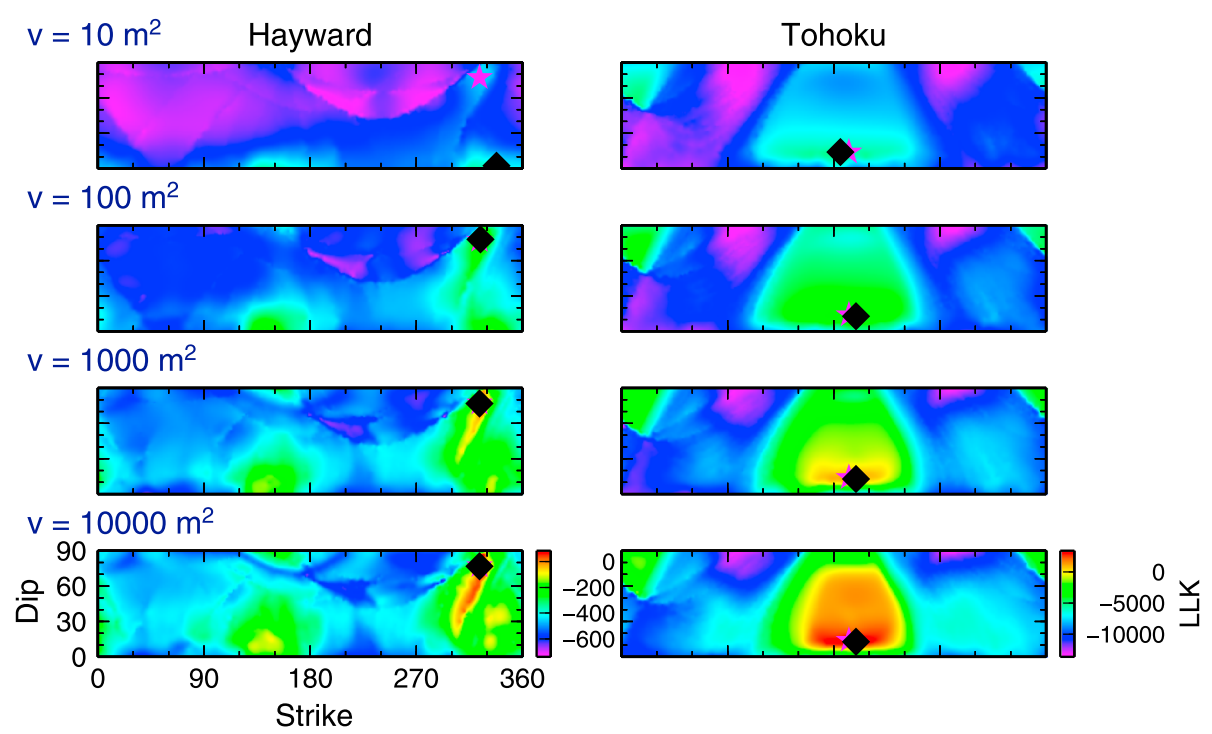

Figure 7. Effects on inversion results for fault geometry of varying $v$. The log of the marginal likelihood is plotted for all values of strike and dip for various values of $v$ for two simulated earthquakes. The most likely fault geometry found by inversion is marked with a black diamond. The input fault geometry is shown with a pink star. 


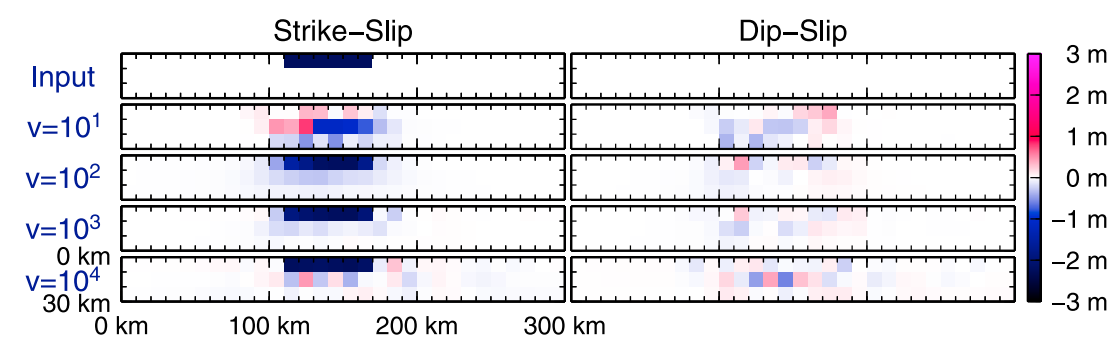

Figure 8. Effects on inversion results for slip of varying $v$. The mean posterior estimate for the (left) strike-slip and (right) dip-slip components of motion are shown for a simulated $M_{w} 7$ Hayward fault earthquake. For reference, the input slip model is shown in the top row. Variance, $v$, is in units $m^{2}$.

for all components yields equally poor results (Figure 4, second row). To be able to resolve the fault geometry, we found that it was necessary to weight each data point by how close the observation location is to the source. For example, by assigning a variance to each observation of $10^{-5} \frac{\mathrm{m}^{2}}{\mathrm{~km}^{2}} \operatorname{diag}\left\{r^{2}\right\}$ where $r$ is the epicentral distance of each observation location in kilometers (Figure 4, third row). However, as the rupture grows, weighting stations by their epicentral distance might be a poor metric for a station's final effective source-receiver distance especially if the earthquake ruptures toward the station or if the centroid of slip is located far from the rupture's point of initiation. The closer a receiver is to a locus of slip, the larger the displacement at that receiver. Thus, the magnitude of the observed surface offsets can themselves be used as a proxy for proximity to the rupture. While this approach has the disadvantage that a station reporting a large spurious excursion will have its contribution to the inversion increased, it has the significant advantage that the station weighting dynamically updates as the rupture evolves. In fact, the results for an inversion for the geometry of the Tohoku-oki rupture using an expression for $\mathbf{D}$ that is inversely proportional to the observed data amplitude (Figure 4, fourth row) are even better than the results using a weighting scheme based on epicentral distance, and so we adopt the displacement-amplitude-based form of $\mathbf{D}$ for the inversions presented in the remainder of this paper.

Our inferred fault geometry (equation (5)) and slip distribution (equation (7)) depend on four parameters: $a$ and $d$ (the priors on the regression variance) and $\mathbf{m}$ and $\mathbf{V}$ (the prior mean and covariance of the slip model, respectively). To explore the influence of these four parameters, we conducted a series of synthetic tests using the network geometries of the Hayward fault simulation and Tohoku-oki earthquake. Our input slip models are uniform with length and width proportional to the magnitude of the source using the scaling relationships of Wells and Coppersmith [1994]. We added realistic noise based on the characteristic noise spectra of Langbein and Bock [2004] to our synthetic displacement time series. The noise spectra included $60 \mathrm{~s}$ and $300 \mathrm{~s}$ of random walk noise for the Hayward fault and Tohoku-oki simulations, respectively.

We essentially have no a priori information on what the regression variance should be. But the influence of $a$ and $d$ decrease as they approach zero. As can be seen in Figure 5, once $a$ and $d$ are set to sufficiently small values, changing their value has little effect on the inversion. (The effect of varying $a$ and $d$ is seen most clearly in the inversion results for fault orientation and the moment magnitude of the source.) So any small values for these parameters may be safely used.

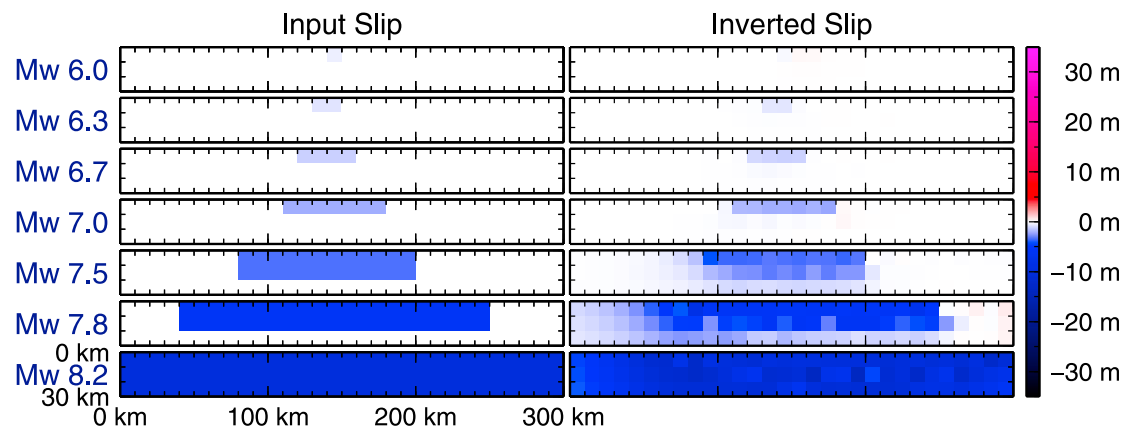

Figure 9. Quality of inversion results for slip as a function of earthquake size. The input slip model and the mean posterior estimate for the strike-slip component of motion are shown for a number of simulated Hayward fault events. 

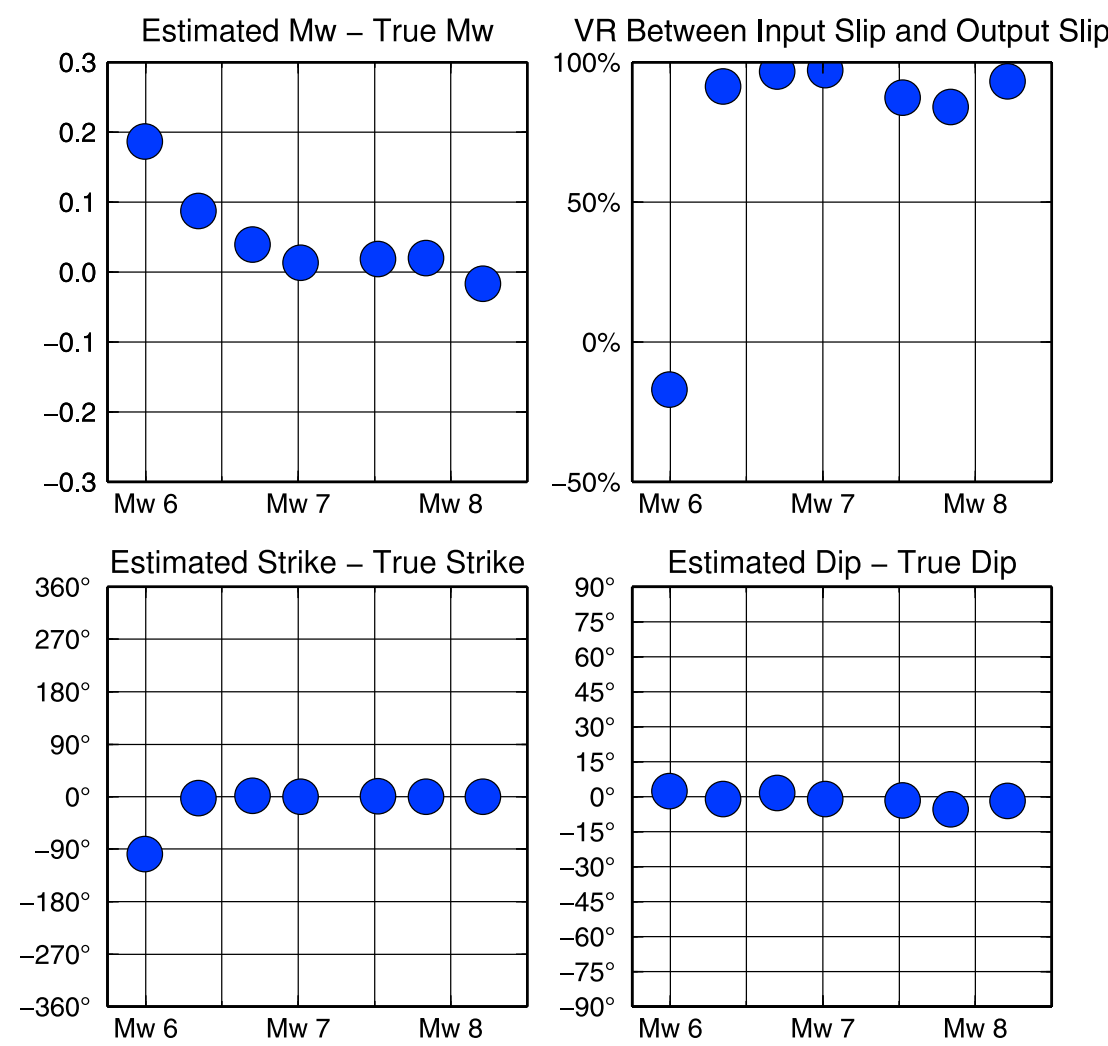

Figure 10. Quality of inversion results as a function earthquake size. The differences between the output moment magnitude, slip model, fault strike, fault dip, and the input values for each of these quantities are shown for a number of simulated Hayward fault earthquakes which vary in magnitude.

Our prior distribution on $\operatorname{slip}, \mathcal{N}(\mathbf{m}, \mathbf{V})$, represents the distribution of possible values of slip for each patch in our source model. Since we do not know a priori which direction the fault will slip (and thus have no a priori knowledge of the sign of $\mathbf{m}$ ), we assume $\mathbf{m}=\mathbf{0}$. Furthermore, we do not have a priori information on either the spatial distribution of the rupture's asperities (where larger or smaller amounts of slip will occur) or the spatial covariances of the slip distribution. Thus, we will only consider isotropic covariance matrices of the form $\mathbf{V}=v \mathbf{I}_{\mathbf{p}}$, where $\mathbf{I}_{\mathbf{p}}$ is the $p$ dimensional identity matrix.

From a practical standpoint, our prior PDF, $\mathcal{N}\left(\mathbf{0}, v \mathbf{I}_{\mathbf{p}}\right)$ effectively acts as a minimization constraint where slips whose magnitude is large compared to $v$ are penalized. Thus, for a given value of $v$, the minimization constraint will be stronger for larger earthquakes. Since we do not want our inversion to falsely force the solution to have smaller slips (and thus smaller implied moment magnitudes) than the true source process being observed, we want to make $v$ sufficiently large to allow slips as large as those expected for great earthquakes. However, we also want to ensure that the inversion is well constrained for both large and moderate earthquakes. In Figures 6-8, we plot the results of inversions for various values of $v$ for a simulated $M_{w} 7$ Hayward fault rupture and a simulated $M_{w} 9$ Tohoku-oki rupture, which roughly corresponds to the range of earthquake magnitudes we are most interested in modeling. We find that setting our prior variance, $v$, to something on the order of $1000 \mathrm{~m}^{2}$ yields the best results. This a priori constraint is equivalent to saying that we expect with $95 \%$ confidence there to be $62 \mathrm{~m}$ of slip or less for each component of motion on each fault patch. While $v=1000 \mathrm{~m}^{2}$ was chosen empirically by comparing results from inversions with different values of $v$, this seems to be a reasonable choice. For comparison, the maximum slip for the $M_{w} 9.0$ Tohoku-oki earthquake, which is the fourth largest earthquake observed worldwide since 1900 (U.S. Geological Survey, http://earthquake.usgs.gov/earthquakes/world/10_largest_world.php) and thus an example of some of the largest fault slips we might plausibly observe, is less than $80 \mathrm{~m}$ (S. E. Minson et al., Bayesian inversion for finite fault earthquake source models II — The 2011 great Tohoku-oki, Japan earthquake, submitted to Geophysical Journal International, 2014). 


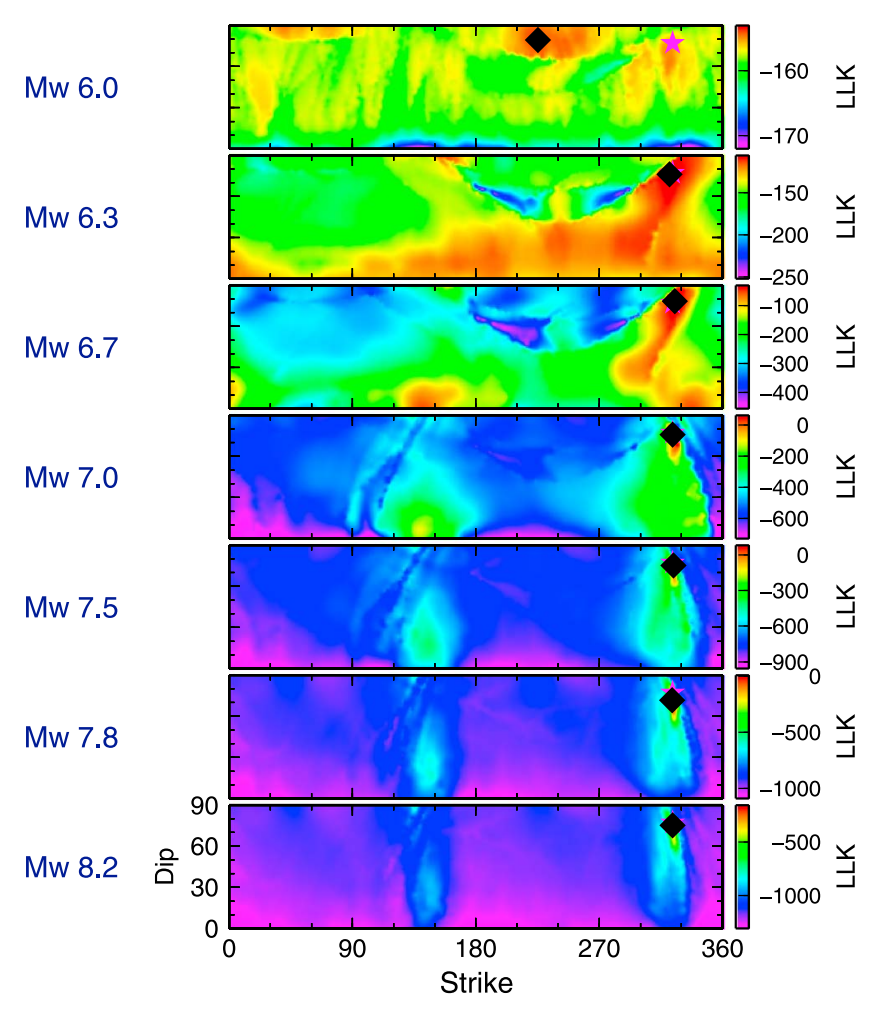

Figure 11. Quality of inversion results for fault geometry as a function earthquake size. The log of the marginal likelihood is plotted for all values of strike and dip for various values of input $M_{w}$ for a Hayward fault simulated earthquake. The most likely fault geometry found by inversion is marked with a black diamond. The input fault geometry is shown with a pink star.

Finally, we are interested in determining what range of earthquakes we can successfully model in real time. Of particular interest is determining the smallest magnitude earthquake we can model since, given the noisiness of real-time $1 \mathrm{~Hz}$ GPS data, the signal-to-noise ratio will be low for even moderate magnitude

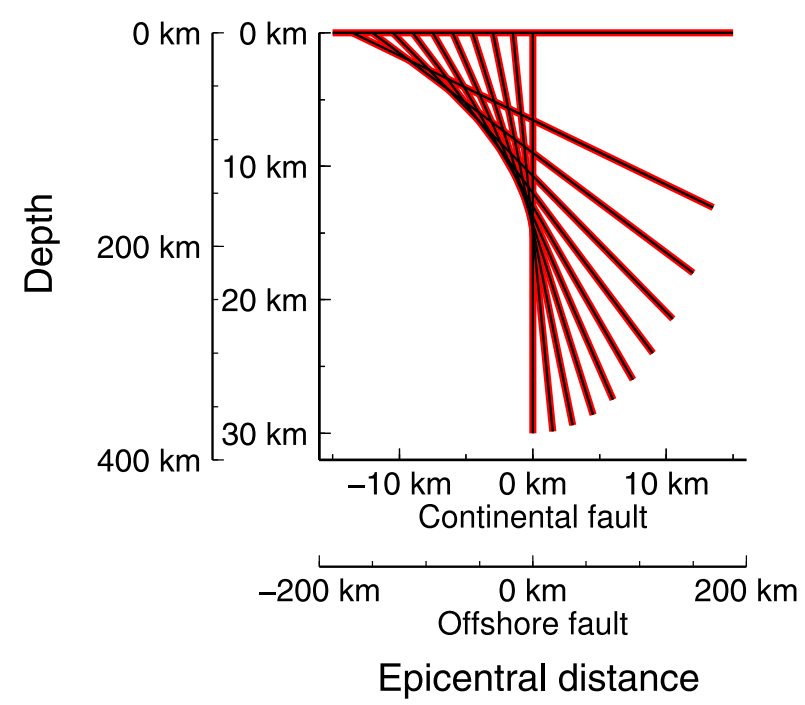

Figure 12. Array of potential geometries for a fault whose updip limit is the free surface. This variation is plotted for a $30 \mathrm{~km}$ wide fault (our assumed width for continental faults) (inner axes) and a $375 \mathrm{~km}$ wide fault (our assumed width for offshore faults) (outer axes) using equally spaced samples of $\cos (\delta)$. earthquakes at close range. Toward this end, we conducted a series of inversions using synthetic observations for a uniform Hayward fault rupture of varying magnitude. The results shown in Figures 9-11 suggest that real-time $1 \mathrm{~Hz}$ GPS data cannot be used to constrain the geometry and slip distribution of earthquake ruptures for events smaller than approximately magnitude 6.5 even if the event is directly beneath a dense network of instruments.

\section{Application}

To test the performance of our proposed real-time finite fault slip inversion approach, we applied the BEFORES inversion methodology of section 2 to $1 \mathrm{~Hz}$ kinematic GPS data from the $2011 M_{w} 9.0$ great Tohoku-oki earthquake, the $2003 M_{w} 8.3$ Tokachi-oki earthquake, and a simulated $M_{w} 6.76$ earthquake on California's Hayward fault. This is a small set of test events, but it does include both continental ruptures and offshore 

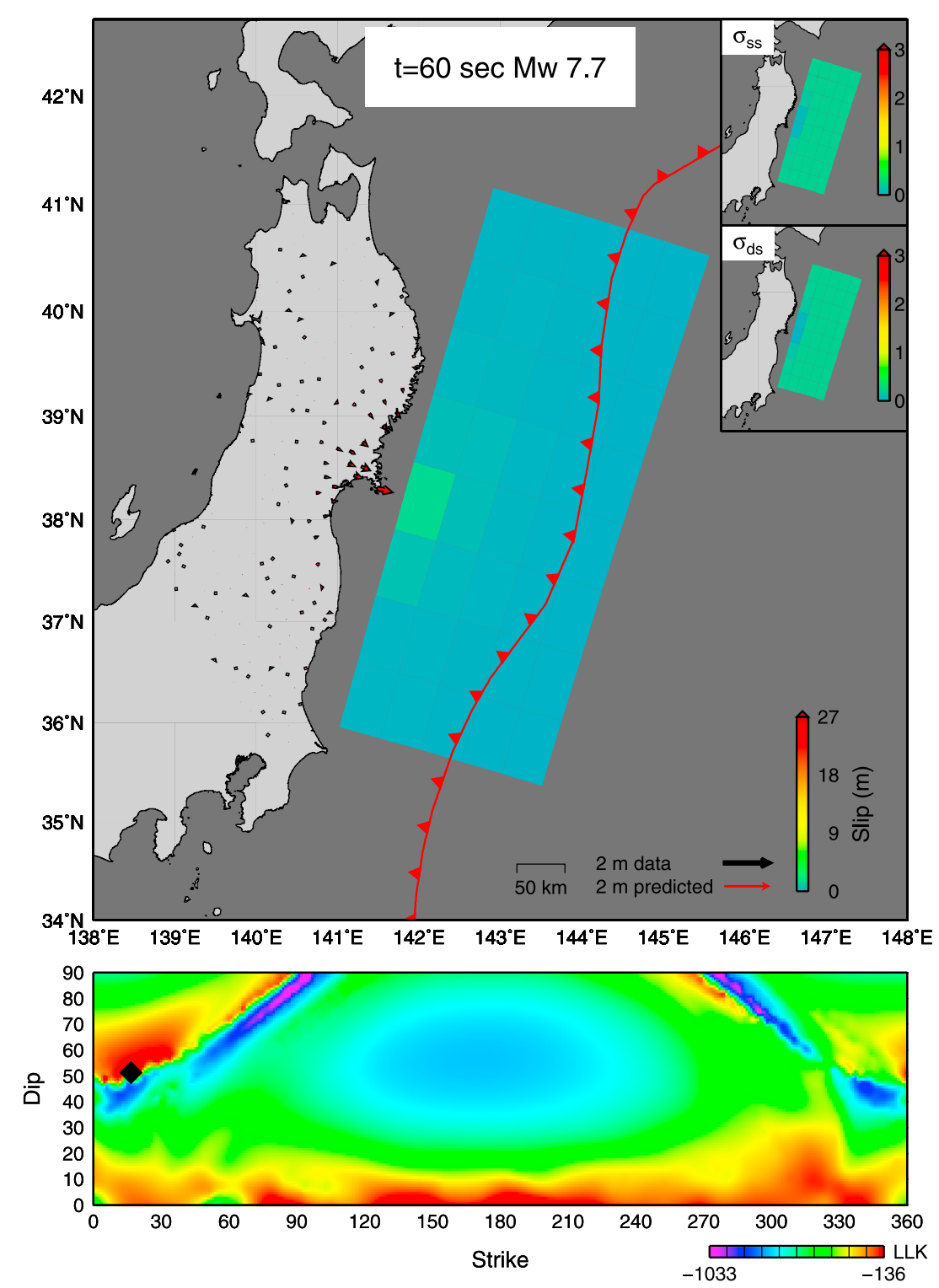

Figure 13. Result of semianalytical Bayesian real-time finite fault inversion for the Tohoku-oki earthquake at $60 \mathrm{~s}$ after the origin time. See text for explanation.

subduction zone events ranging over 3 orders of moment magnitude. The GPS data processing strategies used for these three events are all different and represent a wide range of different processing schemes. The Tohoku-oki data are not real time but are instead a best-case-scenario solution using refined orbits and precise point positioning processing. (See S. E. Minson et al., submitted manuscript, 2014, for details.) High-rate data from the GPS Earth Observation Network (GEONET) were not being processed in real time when the 2003 Tokachi-oki occurred, so our time series for that event are a recreation of what real-time processing might have looked like: it is a network solution based on ultrarapid orbits with a network adjustment made for each epoch [Crowell et al., 2009, 2012]. We added to the Hayward fault scenario synthetic time series noise generated from an empirical analysis of observed noise spectra from actual San Francisco Bay Area real-time high-rate GPS data [Langbein and Bock, 2004]. The positions of each noisy time series were then differenced with the simultaneous position estimate for a reference station, so that our final synthetic data simulate actual noise characteristics of real-time high-rate differential GPS positions, which is the type of real-time solutions currently generated operationally in the Bay Area. Given the moderate magnitude of the Hayward fault simulation and our very high amplitude (but realistic) noise, the resulting time series are the noisiest of the three events studied. 

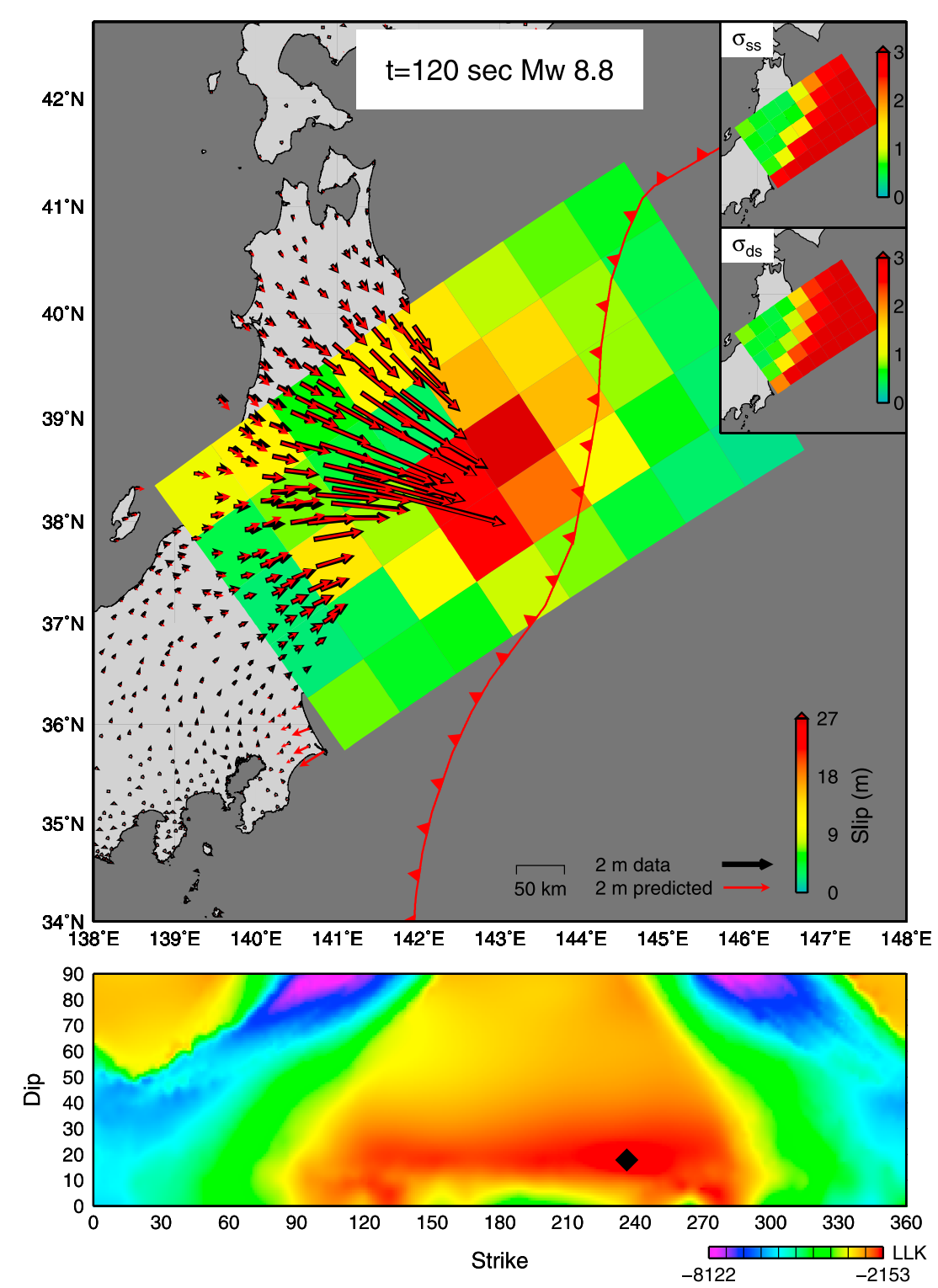

Figure 14. Result of semianalytical Bayesian real-time finite fault inversion for the Tohoku-oki earthquake at $120 \mathrm{~s}$ after the origin time. See text for explanation.

At each time $t$ (where $t$ is measured relative to the origin time), we determined the most probable fault geometry by evaluating the marginal likelihood (equation (5)) for 5000 random samples of strike and dip. As discussed earlier, we chose the mean and variance of the prior PDF on slip to be $\mathbf{m}=\mathbf{0}$ and $\mathbf{V}=1000 \mathrm{~m}^{2} \mathbf{I}_{\mathbf{p}^{\prime}}$ respectively. In section 4 , we demonstrated that any small value for $a$ and $d$ will minimize their influence on the inversion. So we selected $a=d=0.02$. Also, based on the results in section 4 , we used a diagonal data covariance matrix whose nonzero elements are given by $D_{i i}=\left(\sigma_{d} / y_{i}\right)^{2}$, where $y_{i}$ is the $i$ th element of a vector of estimated three-component surface offsets and $\sigma_{d}=0.3$.

For each earthquake, we test our inversion methodology on both the raw time series and on data which have been smoothed using an EWMA (equation (11)) for a variety of decay rates. The offsets, $\mathbf{y}$, that we used for our inversion at each point in time, $t$, are calculated by taking the difference between the station's position (smoothed or not) at time $t$ and its position at the origin time. Rather than select which stations to include in any given inversion based on some kind of triggering algorithm, we included in the inversion at time $t$ the data from all stations located at distances less than $(6 \mathrm{~km} / \mathrm{s}) t+10 \mathrm{~km}$. $(6 \mathrm{~km} / \mathrm{s}) t$ is an estimate of how far the $P$ waves have traveled at time $t$, and the additional $10 \mathrm{~km}$ allows for the pos- 

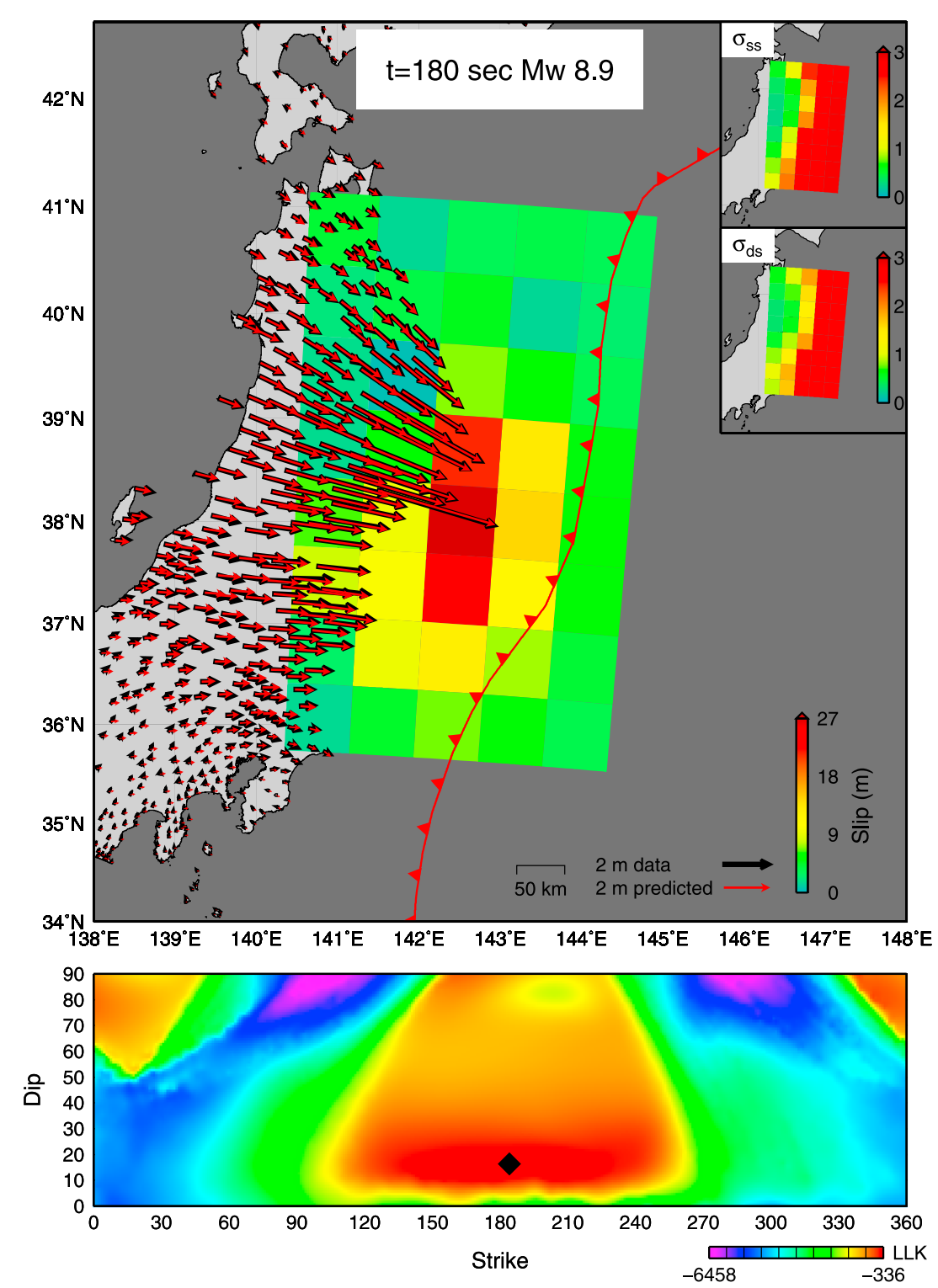

Figure 15. Result of semianalytical Bayesian real-time finite fault inversion for the Tohoku-oki earthquake at $180 \mathrm{~s}$ after the origin time. See text for explanation.

sibility that the hypocenter was mislocated by up to $10 \mathrm{~km}$ leading to the $P$ waves arriving earlier. This approach includes information from all stations that may have seen any kind of displacement from the rupture and only eliminates data from stations which could not have received any signal yet. We use data from all stations, no matter how small the observed displacement. Thus, stations with zero displacement are used to help constrain the inversion (once they are in $P$ wave range of the source), although their contribution to the inversion is necessarily diminished by our data weighting scheme.

The two subduction zone events, Tohoku-oki and Tokachi-oki, are located well offshore, and we expect to have poor spatial resolution on the slip distribution of those earthquakes. Therefore, we use a rather spatially coarse fault parameterization: a $5 \times 8$ grid of square patches that are $75 \mathrm{~km}$ long on a side. The Hayward fault rupture is located directly under our network of stations, so we use a finer grid composed of $10 \mathrm{~km}$ long square patches. We chose a grid 3 patches wide and 30 patches long because we do not expect faults to be wider than about $30 \mathrm{~km}$ in a continental setting, and a $300 \mathrm{~km}$ long fault model is probably sufficient to describe a major rupture on a continental fault. 

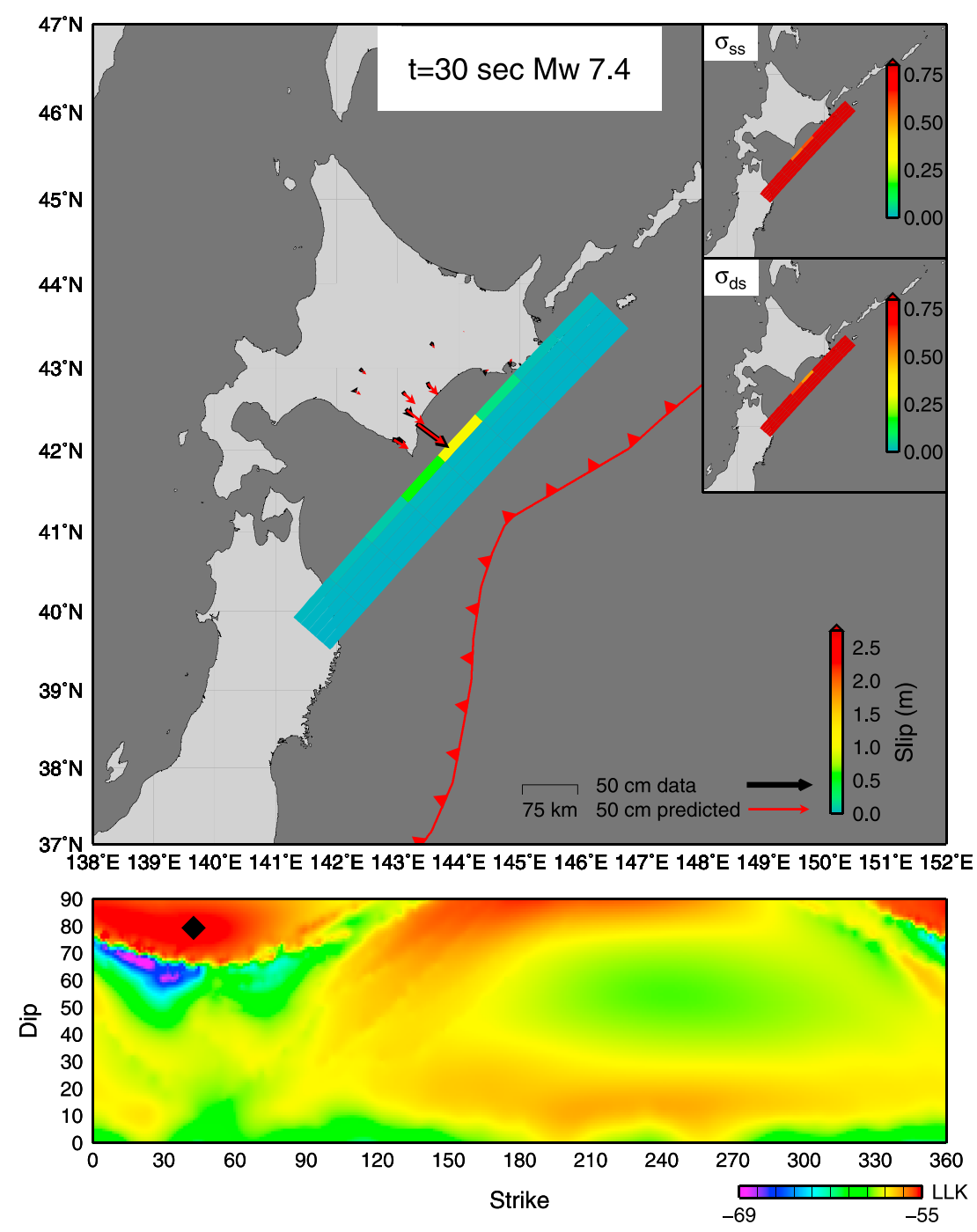

Figure 16. Result of semianalytical Bayesian real-time finite fault inversion for the Tokachi-oki earthquake at $30 \mathrm{~s}$ after the origin time. See text for explanation.

For every fault geometry, we center the fault on the epicenter of the rupture. Since many EEW systems currently do not report hypocentral depths in real time, we assume that the depth of the fault is unknown. In the absence of any information about the depth of the earthquake, it seems most reasonable to assume that the top of the fault is the Earth's surface. So for each fault geometry we generate, we shift it vertically so that the top edge of the fault intersects the surface (Figure 12). We then calculate Green's functions for each fault patch using the Okada [1985] solution for a rectangular dislocation in a homogeneous elastic half-space.

The results of our inversion for the geometry and slip distribution of the 2011 Tohoku-oki earthquake using displacement estimates taken at 1-3 min after the origin time are presented in Figures 13-15. In Figures 13 (top), 14 (top), and 15 (top), our estimated static offsets (which are the data we use in our inversion) are shown with black vectors. These offsets are the difference between the observed positions (smoothed using an EWMA with $\lambda=0.1$ ) of each GPS station at this second (60 s after the origin time in Figure 13, $120 \mathrm{~s}$ in Figure 14, and $180 \mathrm{~s}$ in Figure 15) and the position of the station at the origin time. Figures 13 (bottom), 14 (bottom), and 15 (bottom) show the value of the $\log$ marginal likelihood, $\ln p(\mathcal{D} \mid \mathcal{M})$, as a function of the strike and dip of the fault plane. The fault orientation that maximizes $p(D \mid \mathcal{M})$ is marked with a black diamond. The mean of the posterior PDF on slip given the fault geometry marked with the black diamond is shown in the top plot, and the predicted observations from this slip model are shown with red vectors. The magnitude listed in the figure was calculated from the slip model assuming an elastic shear modulus of $30 \mathrm{GPa}$. The posterior standard deviation of the strike-slip and dip-slip components of slip on the fault 

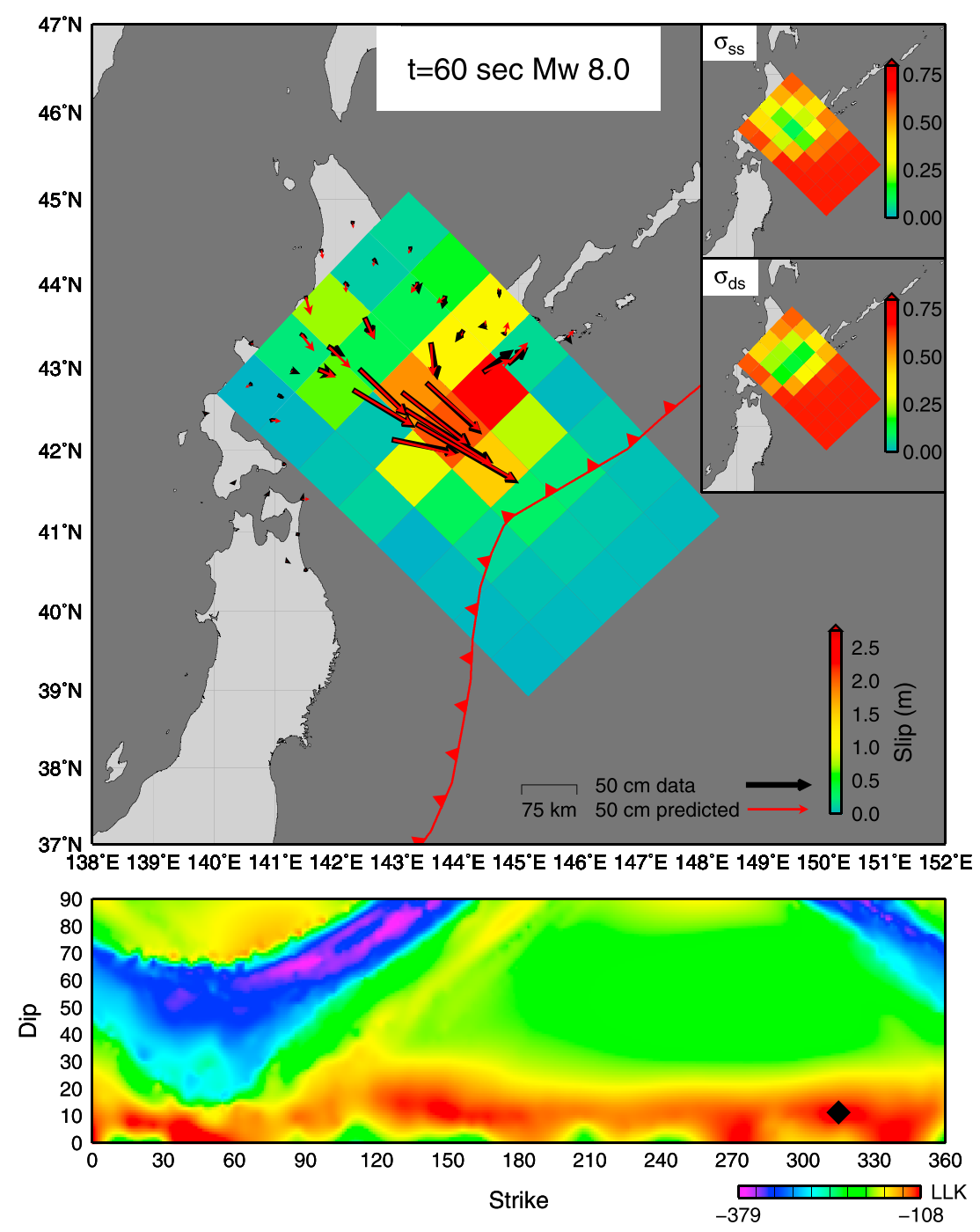

Figure 17. Result of semianalytical Bayesian real-time finite fault inversion for the Tokachi-oki earthquake at $60 \mathrm{~s}$ after the origin time. See text for explanation.

( $\sigma_{\mathrm{ss}}$ and $\sigma_{\mathrm{ds}}$, respectively) are shown in insets in the top right corner. (Uncertainties are shown in units of meters.) Note that the number of stations used in the inversion increases from Figures 13 to 14. This is because more stations are within $P$ wave range of the hypocenter at $2 \mathrm{~min}$ after the origin time than at $1 \mathrm{~min}$ after the rupture begins. Inversion results for the 2003 Tokachi-oki earthquake and the Hayward fault simulation are given in Figures 16-18 and Figures 19-21, respectively. All of the results shown are from inversions where the displacement time series were smoothed by an EWMA with $\lambda=0.1$. Full results at all times, $t$, for all three earthquakes as well as additional inversions with unfiltered and more strongly filtered data are presented in the supporting information.

From these figures, it is apparent that for all three earthquakes it takes longer to constrain the strike and dip of the fault plane than it does to estimate the earthquake magnitude. In fact, early in the rupture, any rupture is so spatially compact that it is indistinguishable from a point source, and the inversion is unable to accurately determine which of the two conjugate planes is the true fault plane and which is the auxiliary plane. This can be seen more clearly in Figure 22. We can conclude that the inversion results for moment magnitude are robust and also rapid if either unsmoothed or minimally smoothed time series are used (black and red lines in Figure 22). However, too much smoothing (e.g., blue lines in Figure 22) is clearly detrimental to the efficiency of the inversion, producing a significant and undesirable lag into the estimates of the strike, dip, and moment magnitude. While either unsmoothed or minimally smoothed data produce 

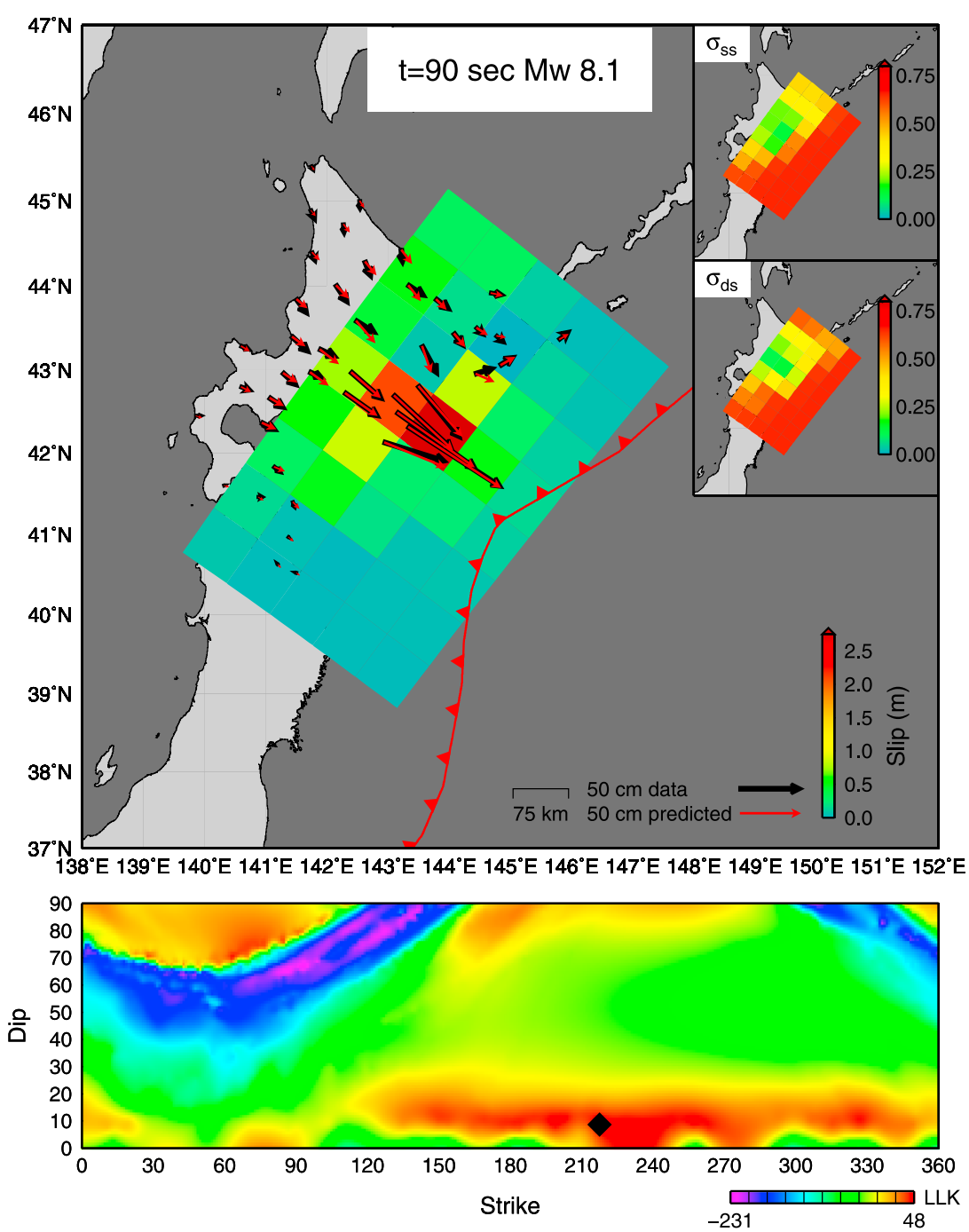

Figure 18. Result of semianalytical Bayesian real-time finite fault inversion for the Tokachi-oki earthquake at $90 \mathrm{~s}$ after the origin time. See text for explanation.

timely inversion results for moment magnitude, the estimate for strike and dip seems to converge more rapidly if some smoothing is applied to the data.

Most importantly, if we consider the evolution of the moment magnitude estimate for the Tohoku-oki earthquake (top right subplot in Figure 22), we see that using real-time high-rate GPS to estimate magnitude in real time provides a significant improvement over magnitude estimates from existing EEW systems like the one in use during the Tohoku-oki earthquake. For the largest earthquakes, we expect EEW magnitude estimates based the first few seconds of seismic data to saturate [e.g., Wu and Zhao, 2006]. Indeed, the seismic-only Japan Meteorological Agency (JMA) EEW system misidentified the Tohoku-oki earthquake as a magnitude 8 event. However, the GPS-based $M_{w}$ estimate, which can be obtained just as quickly as the seismic estimate (once the hypocenter is known) does not saturate and correctly identifies the Tohoku-oki earthquake as an $M_{w} 9$ event.

We also see in Figures 13-21 that the spatial distribution of posterior uncertainties on slip is controlled by the spatial distribution of stations. For the offshore subduction zone events, slip on patches nearest the coast are best resolved. In the case of the Hayward fault scenario earthquake, where the fault geometry extends in both directions along strike beyond the network of stations, slip is best resolved in the central part of the fault that is beneath the network of stations. Within this central region, slip on the shallow patches is better resolved than deep patches, as is expected. Although the absolute magnitude of the 

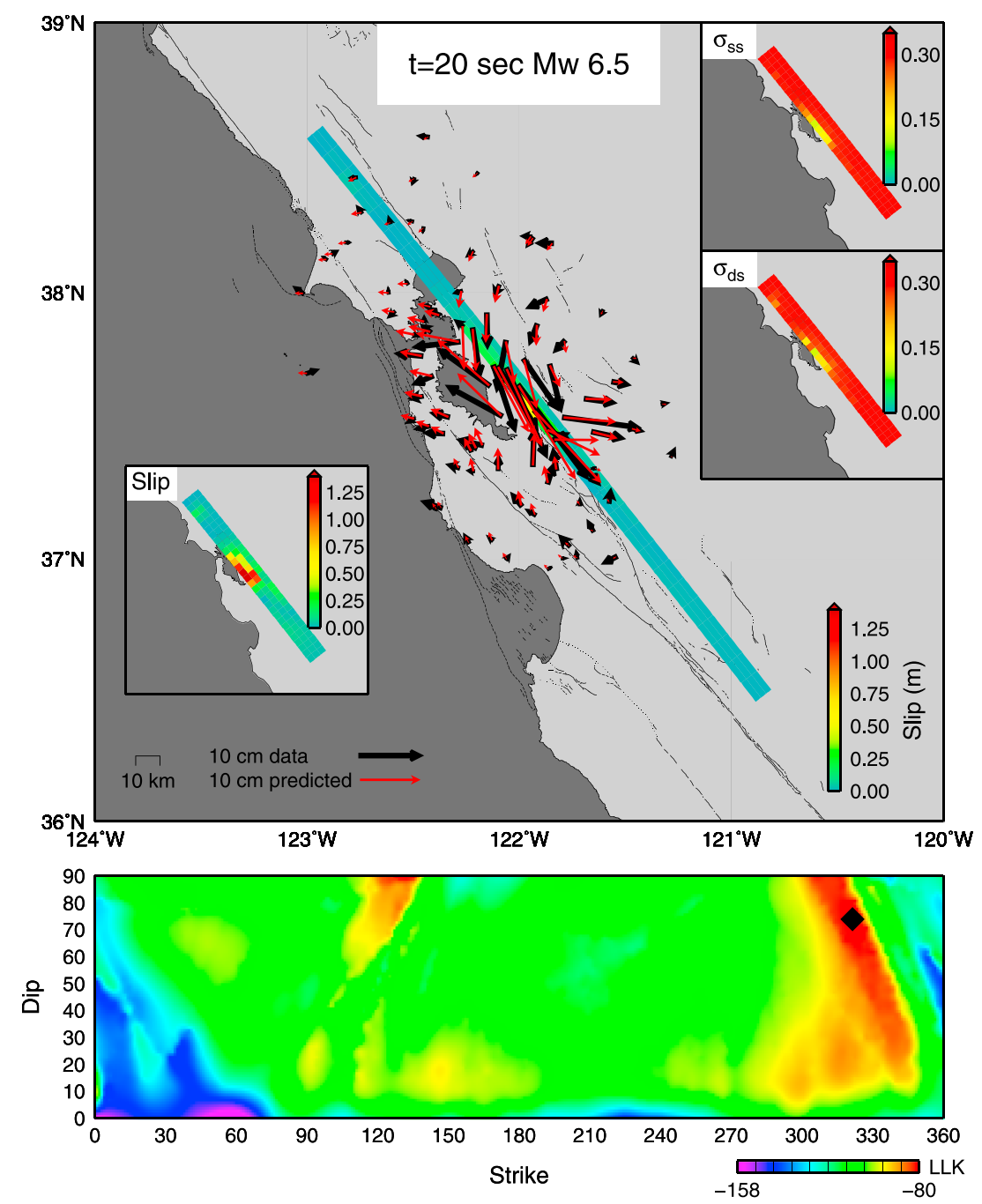

Figure 19. Result of semianalytical Bayesian real-time finite fault inversion for the Hayward fault simulation at $20 \mathrm{~s}$ after the origin time. See text for explanation. In the insets, the fault is plotted as horizontally dipping for ease of viewing.

uncertainties in Figures 13-21 tend to be small, it should be noted that each subfault is large and thus the inferred slip amplitude (which is an average over the area of each subfault) tends to be lower than in models with smaller subfaults because there is an increased chance of averaging a spatially small, high slip amplitude asperity with an area with less slip. When these uncertainties are viewed relative to the posterior mean slip on each patch (see supporting information), these are in fact quite large relative to the inferred slip. This implies that the solution has significant uncertainties, which is what we would expect for a simple fault model, with no a priori knowledge of the fault geometry, constrained by a limited number of noisy observations.

\section{Operational Considerations}

In this section, we will outline the steps necessary to implement our real-time Bayesian finite fault slip inversion methodology, BEFORES. First, it should be noted that our inversion requires initialization with an estimate of the earthquake's location. Although we would prefer to center our fault model on the event's hypocenter, most EEW systems currently in operation report epicenters only, meaning that the user will have to decide at what depth to place the fault. In this paper, we have always fixed the depth so that the top of the fault intersects the surface. However, in principle, the depth of the event could be solved for as part of the inversion by evaluating the marginal likelihood for many choices of hypocentral depth as well as all possible fault orientations. 

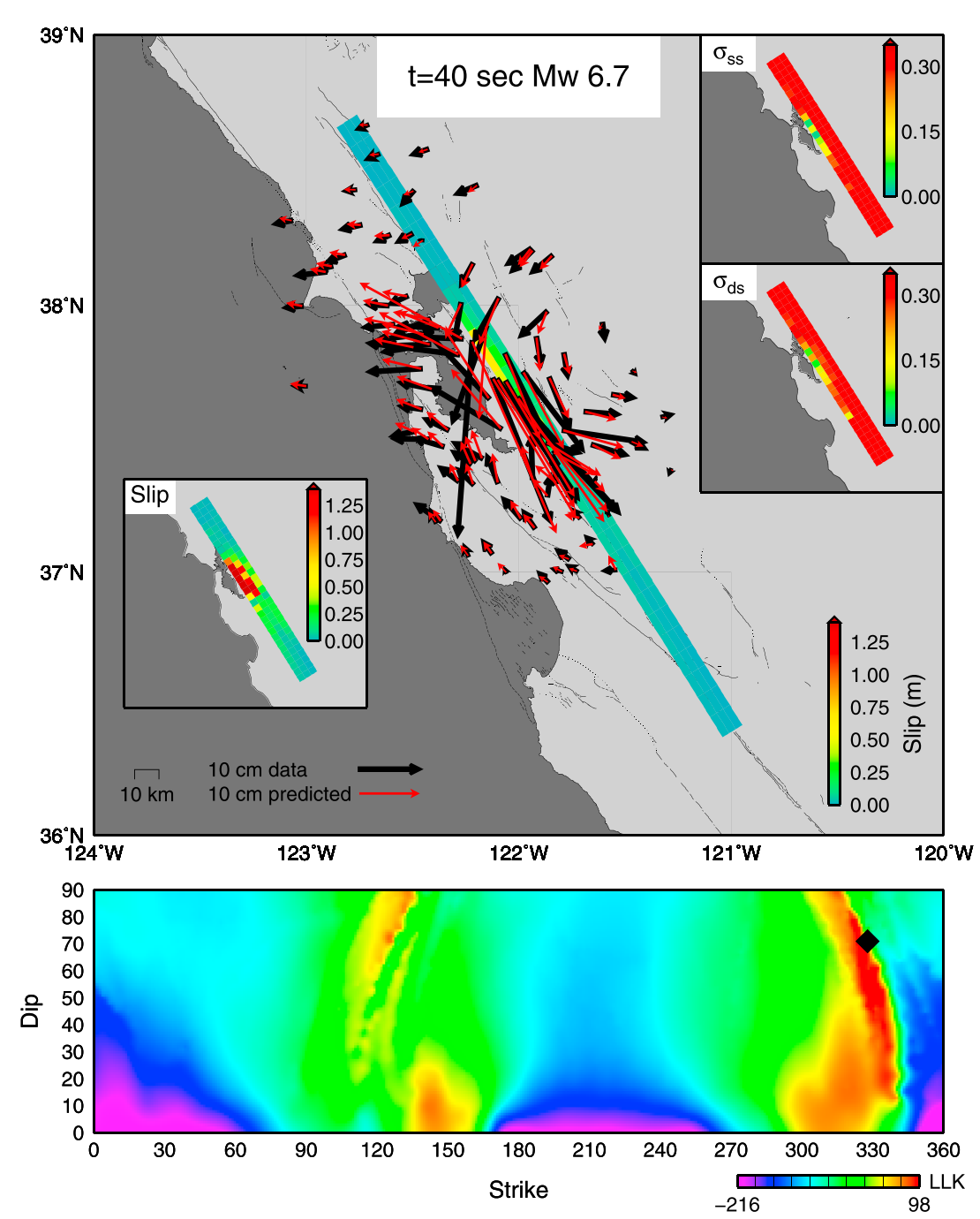

Figure 20. Result of semianalytical Bayesian real-time finite fault inversion for the Hayward fault simulation at $40 \mathrm{~s}$ after the origin time. See text for explanation. In the insets, the fault is plotted as horizontally dipping for ease of viewing.

Second, once a location has been specified, we must choose the shape and size of the discretized fault plane. For the performance tests presented in this paper, we have used a rather crude scheme in which we have used a coarse grid that is broad in both directions for offshore (and thus poorly resolved) earthquakes and a fine grid that is only $30 \mathrm{~km}$ wide for continental earthquakes. The best practice would be for operators to predetermine the spatial resolution at all potential source locations given their network of instruments and then, when an earthquake occurs and its location is identified, use their library of model resolution results to look up the appropriate grid spacing for that source location. Similarly, network operators might determine in advance what the maximum rupture size could be for an earthquake at any location by constraining the fault length based on the lengths of mapped faults in that region and constraining the fault width based on the observed depth extent of seismicity or geodetic models of locking depth. However, given that solving for slip on the fault plane is the analytical part of the simultaneous inversion for slip and fault geometry, there is relatively little computational expense associated with increasing the number of fault patches and the inversion is free to put zero slip on patches which did not move. So operators should be encouraged to use the largest physically plausible spatial dimensions so as not to underestimate the actual size of the rupture.

Third, the prior PDF on the slip inversion needs to be chosen. But this can be done with a few rules of thumb. We recommend $\mathbf{m}=\mathbf{0}, \mathbf{V}$ large and isotropic, and $a$ and $d$ small to produce stable inversion results over a wide range of slip amplitudes. 

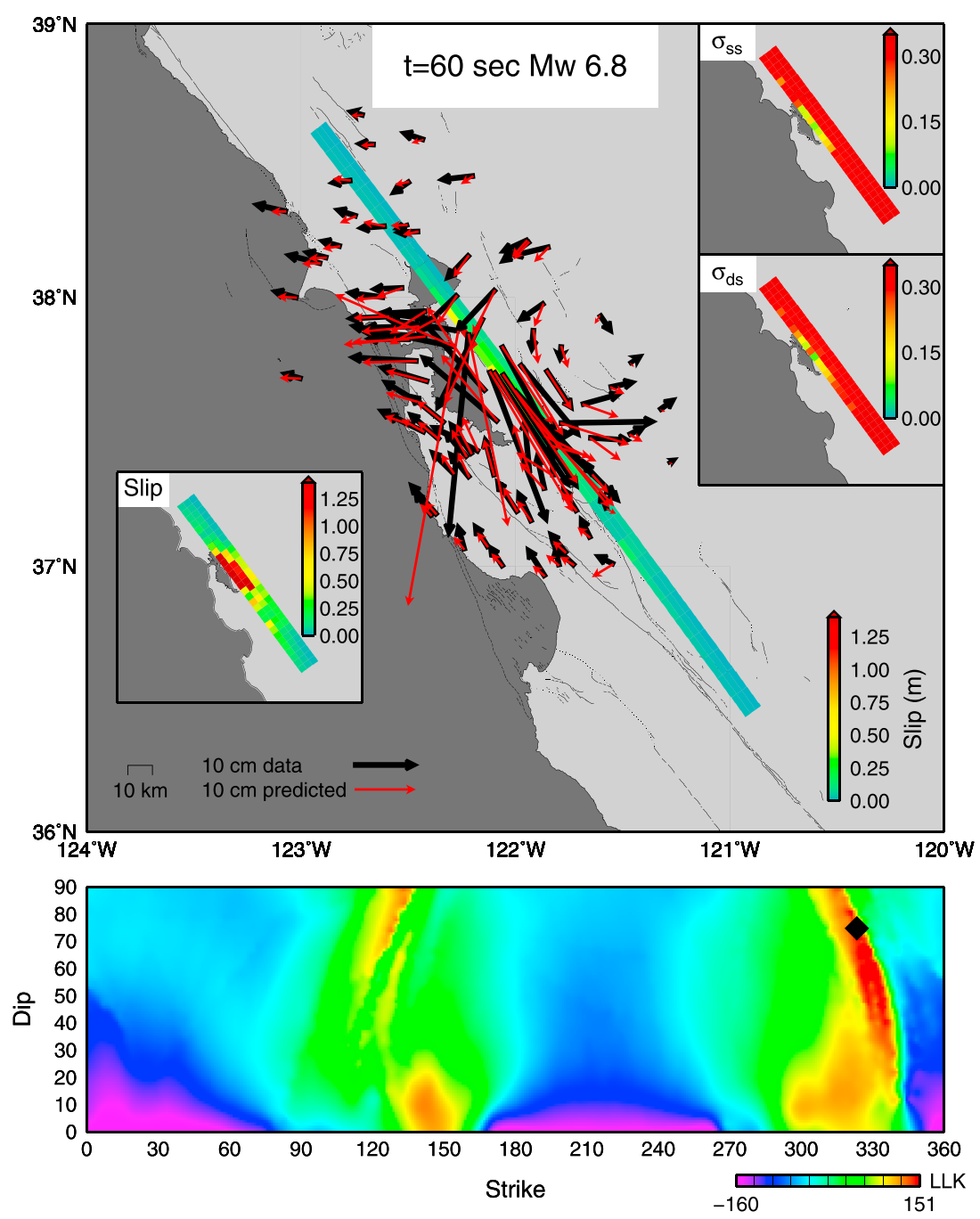

Figure 21. Result of semianalytical Bayesian real-time finite fault inversion for the Hayward fault simulation at $60 \mathrm{~s}$ after the origin time. See text for explanation. In the insets, the fault is plotted as horizontally dipping for ease of viewing.

Finally, the user will still need to specify the data, $\mathbf{y}$, Green's functions, $\mathbf{X}$, and data covariance matrix, $\mathbf{D}$ for the inversion. In this paper, we used for our data, $\mathbf{y}$, quasi-static surface displacements which we estimated from high-rate GPS data. But other information about the static displacement field, such as borehole strain data, could be used instead or in addition. We have already discussed our preferred choice for $\mathbf{D}$ in section 4. Our inversion results using Green's functions for a simple elastic half-space seem of sufficient quality. Of course, using Green's functions for more realistic Earth structures would be expected to give improved results. In any case, a library of Green's functions should ideally be calculated in advance and stored for use on demand when an earthquake occurs.

From Figure 22, we can see that accurate moment magnitude estimates are obtained most quickly from the least smoothed data, but some data smoothing may be required to stabilize the inversion for strike and dip. (This behavior is most apparent for the Hayward fault simulation.) In an operational context and if sufficient computational resources are available, it might be preferable to run multiple inversions simultaneously: one using the unfiltered time series in order to determine $M_{w}$ as quickly as possible and one using smoothed data to produce more robust geometry solutions. If only one inversion is to be made, we recommend using a small amount of smoothing (e.g., an EWMA with $\lambda=0.1$ ). 

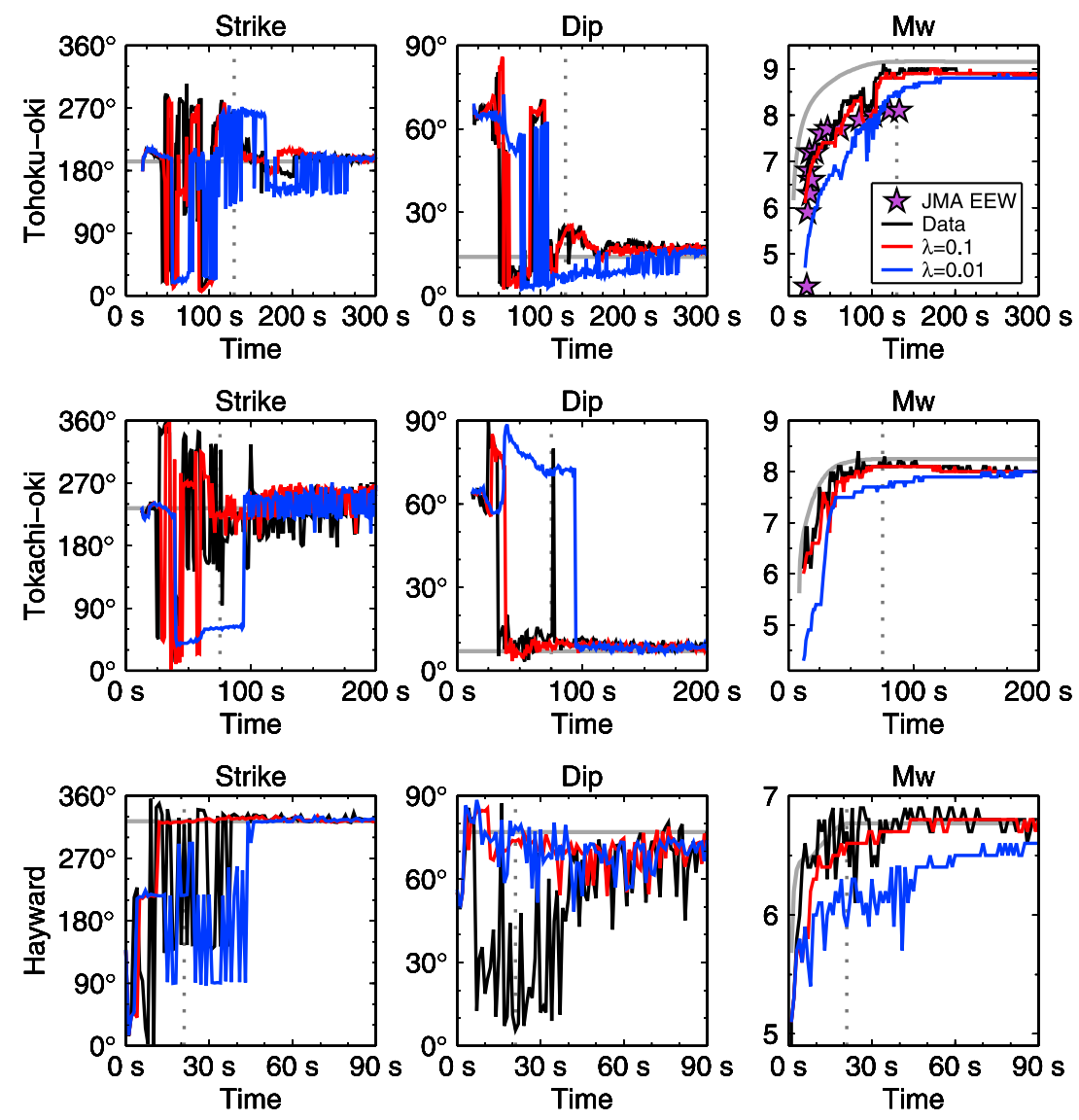

Figure 22. Comparison of strike, dip, and moment magnitude estimates from inversions using raw data (black) to those using data smoothed with an exponentially weighted moving average with decay rates $\lambda=0.1$ (red) and $\lambda=0.01$ (blue). The $M_{j}$ magnitudes reported by the Japan Meteorological Agency (JMA) EEW system are shown with stars. The target values for each quantity are shown with gray lines. The target strike and dip are assumed to be constant throughout the rupture, while the total moment magnitude released increases as a function of time. The evolution of moment magnitude is calculated from the inferred source time functions of the Tohoku-oki earthquake (S. E. Minson et al., submitted manuscript, 2014) and Tokachi-oki earthquake (H. Kanamori, personal communication, 2013), while the magnitude release from the Hayward fault rupture is precisely known because it is a simulation. Vertical dotted lines indicate the total source duration of each earthquake.

\section{Conclusions}

We have outlined a strategy that utilizes the strengths of Bayesian inference to solve for a finite fault distributed slip model on a fault of unknown orientation in real time. The inversions in this paper, in turn, rely on newly available real-time high-rate GPS data. Perhaps the greatest single benefit of this approach is that it can deliver, in real time, estimates of moment magnitude (the single most important source parameter for hazard assessment, EEW, and emergency response) that do not saturate for large earthquakes. However, by engaging the challenging problem of simultaneously determining the rupture geometry and spatial distribution of slip in real time, we can potentially provide much more information about the source than is available from existing EEW systems. Additional information products which could be derived from these real-time finite fault models include focal mechanisms, rupture extent, location of maximum slip, and the location of surface ruptures along with estimated displacements. While the inversion methodology presented in this paper is purely static, by solving for slip at each second (or whatever time interval is desired or practical) during the rupture, we obtain snapshots of the rupture at every moment in time. We then could in principle reconstruct the full rupture evolution from these results. So potentially, these static inversions could be used to estimate kinematic rupture properties such as rupture velocity and directivity. (However, since our inversion is based on a quasi-static representation of the source, any kinematic properties derived from our inversion results would be an approximation.) Furthermore, all of these potential scientific 

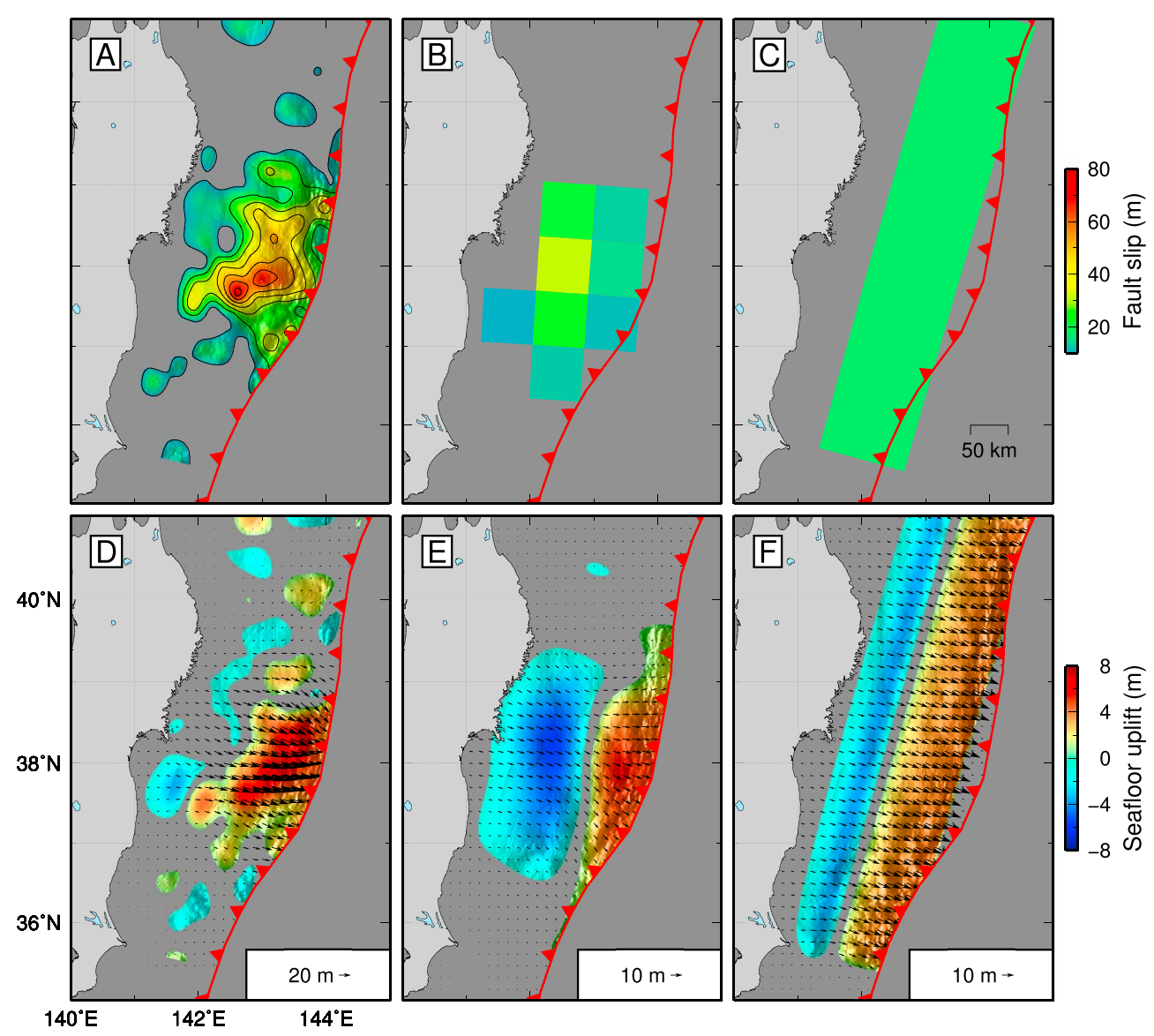

Figure 23. Comparison of slip models and their predicted seafloor displacements for the Tohoku-oki earthquake. (a-c) The spatial distribution of fault slip for a kinematic rupture model (S. E. Minson et al., submitted manuscript, 2014), the results from this study at $180 \mathrm{~s}$ after the origin time (Figure 15), and a rectangular dislocation (length $650 \mathrm{~km}$, width $125 \mathrm{~km}$, focal mechanism from Duputel et al. [2011]), respectively. (d-f) The predicted seafloor displacements for the slip models in Figures 23a-23c, respectively. Seafloor uplift is shown in background color. Horizontal displacements are shown with vectors. Seafloor displacements in Figure 23d were computed using the same layered elastic structure as (S. E. Minson et al., submitted manuscript, 2014), while Figures 23e and $23 \mathrm{f}$ were calculated using a homogeneous elastic half-space. Fault slip less than $10 \mathrm{~m}$ and vertical seafloor motion less than $1 \mathrm{~m}$ are shown transparent.

products could be used, in turn, as inputs into other analyses. These slip models could be used to make high-quality shaking forecasts or could be used as detailed inputs into tsunami propagation models and thus used for tsunami warnings.

To illustrate this last point, in Figure 23 we compare the predicted seafloor displacement from our real-time finite fault inversion for the Tohoku-oki earthquake to the predicted seafloor deformation from naively assuming a uniform source with length and width typical of an $M_{w} 9$ earthquake from Wells and Coppersmith [1994] and to the best inferred seafloor deformation from a kinematic rupture model of the Tohoku-oki earthquake constrained by postprocessed $1 \mathrm{~Hz}$ kinematic GPS time series, static GPS offsets, seafloor geodesy, and both near-field and far-field tsunami data (S. E. Minson et al., submitted manuscript, 2014). While the real-time finite fault slip model is a coarse approximation of the true slip distribution, it does a reasonable job of predicting the seafloor deformation, suggesting that our inversion methodology would be effective for tsunami warning applications.

Bayesian methods are increasingly being used in geophysics, generally, and earthquake seismology, specifically [e.g., Fukuda and Johnson, 2008; Monelli and Mai, 2008; Fukuda and Johnson, 2010; Minson et al., 2013]. However, most work has been directed toward obtaining Bayesian solutions to inverse problems by simulating the posterior PDF using Monte Carlo methods. These numerical techniques are 
computationally expensive and thus not applicable to real-time monitoring. In contrast, in this paper we present a semianalytical Bayesian model which can be computed in real time.

We have examined how best to estimate static offsets from kinematic GPS data. There have been a number of different papers published in which different averaging schemes were used to smooth the observed GPS waveforms [e.g., Allen and Ziv, 2011; Ohta et al., 2012; Crowell et al., 2012; Melgar et al., 2012], sometimes using substantial smoothing to remove any trace of the seismic waves. It should be noted that since the kinematic data will eventually reach a steady-state offset, all methods of averaging out the dynamic wave propagation will eventually converge to the final static offset. The question for the applications explored here is not if a potential offset estimation method will obtain the right estimate but when. Our goal is to identify as much information as possible about the earthquake rupture in real time as the rupture is evolving. Thus, the best way to evaluate these offset estimation techniques is to ask which produces the best inversion solutions the fastest. When it comes to rapidly estimating moment magnitude, it appears that the best data smoothing is none. This is consistent with Wright et al. [2012], who were able to accurately estimate the magnitude of the Tohoku-oki earthquake using offset estimates based on the raw GPS time series. However, the raw time series can make the inversion for strike and dip unstable. So we suggest that some smoothing is desirable to stabilize the inversion for fault geometry.

While the estimated magnitude of the earthquake is robust and steadily grows in value, tracking the actual moment release of the earthquake, the inversion for fault geometry is not stable early in the rupture process because it is impossible to distinguish between the true fault plane and its conjugate until the rupture has grown sufficiently in size that the effects of fault finiteness are significant. Since the inverse problem itself can be calculated in real time at each epoch, the speed with which the inversion stabilizes is not controlled by the computational cost of the inversion. Instead, it is controlled by the duration of the rupture and time required for information to travel from the source to the receivers.

\section{Appendix A: Evidence-Based Model Class Selection}

Muto and Beck [2008] includes a thorough discussion regarding the use of the marginal likelihood, or evidence, $\mathrm{p}(\mathcal{D} \mid \mathcal{M})$ for choosing between different model classes. This process of choice is known as model class selection. To better understand how the evidence acts to identify the optimal model class, let us rewrite Bayes' theorem (equation (2)) to explicitly include the model class, $\mathcal{M}$ :

$$
\begin{aligned}
\mathrm{p}(\boldsymbol{\theta} \mid \mathcal{D}, \mathcal{M}) & =\frac{p(\mathcal{D} \mid \boldsymbol{\theta}, \mathcal{M}) p(\boldsymbol{\theta} \mid \mathcal{M})}{\mathrm{p}(\mathcal{D} \mid \mathcal{M})} \\
& =\frac{\mathrm{p}(\mathcal{D} \mid \boldsymbol{\theta}, \mathcal{M}) \mathrm{p}(\boldsymbol{\theta} \mid \mathcal{M})}{\int \mathrm{p}(\mathcal{D} \mid \boldsymbol{\theta}, \mathcal{M}) \mathrm{p}(\boldsymbol{\theta} \mid \mathcal{M}) \mathrm{d} \boldsymbol{\theta}}
\end{aligned}
$$

Rearranging the terms in equation (A1) yields

$$
\mathrm{p}(\mathcal{D} \mid \mathcal{M})=\frac{\mathrm{p}(\mathcal{D} \mid \boldsymbol{\theta}, \mathcal{M}) \mathrm{p}(\boldsymbol{\theta} \mid \mathcal{M})}{\mathrm{p}(\boldsymbol{\theta} \mid \mathcal{D}, \mathcal{M})}
$$

All PDFs by definition must integrate to one. Thus, we can write

$$
\begin{aligned}
\ln \mathrm{p}(\mathcal{D} \mid \mathcal{M})= & \ln \mathrm{p}(\mathcal{D} \mid \mathcal{M}) \int \mathrm{p}(\boldsymbol{\theta} \mid \mathcal{D}, \mathcal{M}) \mathrm{d} \boldsymbol{\theta} \\
= & \int \ln [\mathrm{p}(\mathcal{D} \mid \mathcal{M})] \cdot \mathrm{p}(\boldsymbol{\theta} \mid \mathcal{D}, \mathcal{M}) \mathrm{d} \boldsymbol{\theta} \\
= & \int \ln \left[\frac{\mathrm{p}(\mathcal{D} \mid \boldsymbol{\theta}, \mathcal{M}) p(\boldsymbol{\theta} \mid \mathcal{M})}{\mathrm{p}(\boldsymbol{\theta} \mid \mathcal{D}, \mathcal{M})}\right] \cdot \mathrm{p}(\boldsymbol{\theta} \mid \mathcal{D}, \mathcal{M}) \mathrm{d} \boldsymbol{\theta} \\
= & +\int \ln [\mathrm{p}(\mathcal{D} \mid \boldsymbol{\theta}, \mathcal{M})] \cdot \mathrm{p}(\boldsymbol{\theta} \mid \mathcal{D}, \mathcal{M}) \mathrm{d} \boldsymbol{\theta} \\
& -\int \ln \left[\frac{\mathrm{p}(\boldsymbol{\theta} \mid \mathcal{D}, \mathcal{M})}{\mathrm{p}(\boldsymbol{\theta} \mid \mathcal{M})}\right] \cdot \mathrm{p}(\boldsymbol{\theta} \mid \mathcal{D}, \mathcal{M}) \mathrm{d} \boldsymbol{\theta}
\end{aligned}
$$

When the evidence is rewritten in this way, we see that it is the difference of two quantities. The first term increases with increasing data fit, $\mathrm{p}(\mathcal{D} \mid \boldsymbol{\theta})$. The second quantity, called the relative entropy, measures how 
different the posterior solution is from our prior PDF. It thus acts as a penalty term, reducing the evidence for model classes which are overly complex or extract too much information from the data. This makes the evidence not only an effective metric for evaluating the relative quality of different model classes but one which is well grounded in the principles of information theory.

\section{Appendix B: Derivation of Normal-Inverse-Gamma Conjugate Prior for Linear Regression With Generalized Error Variance}

For the convenience of the reader, we include the complete derivation of the solution to linear regression with generalized error variance using a normal-inverse-gamma conjugate prior. The notation and proofs are taken from O'Hagan [1994].

We define a generic linear model in matrix notation as

$$
\mathbf{y}=\mathbf{X} \boldsymbol{\beta}+\epsilon
$$

where $\mathbf{y}$ is an $n \times 1$ vector of observations, $\mathbf{X}$ is an $n \times p$ matrix of known coefficients, $\boldsymbol{\beta}$ is a $p \times 1$ vector of parameters, and $\epsilon$ is an $n \times 1$ vector of random errors. The elements of $\epsilon$ are assumed to have zero mean and common covariance $\sigma^{2} \mathbf{D}$, where $\mathbf{D}$ is a known positive definite covariance matrix and $\sigma^{2}$ is an additional parameter. Both the coefficients of our linear regression, $\boldsymbol{\beta}$, and the regression variance, $\sigma^{2}$, are unknown. So the vector of all unknown model parameters can be written as, $\boldsymbol{\theta}=\left(\boldsymbol{\beta}, \sigma^{2}\right)$. Although for the rest of this derivation we will consider generic linear regression problems only, for the inversion described in section 2 we can identify $\mathbf{X}$ as a matrix of Green's functions for a fault with a particular strike and dip, $\mathbf{y}$ as our estimated static offsets, and $\boldsymbol{\beta}$ as a vector containing the strike-slip and dip-slip motions on all fault patches so that $p$ is twice the number of fault patches in our model.

The data likelihood is the multivariate normal distribution:

$$
\begin{aligned}
\mathrm{p}(\mathcal{D} \mid \boldsymbol{\theta}) & =\mathrm{p}\left(\mathcal{D} \mid \boldsymbol{\beta}, \sigma^{2}\right) \\
& =\mathcal{N}\left(\mathbf{X} \boldsymbol{\beta}, \sigma^{2} \mathbf{D}\right) \\
& =\frac{1}{(2 \pi)^{n / 2}\left|\sigma^{2} \mathbf{D}\right|^{1 / 2}} \cdot e^{-\frac{(\mathbf{y}-\mathbf{X} \boldsymbol{\beta})^{T}\left(\sigma^{2} \mathbf{D}\right)^{-1}(\mathbf{y}-\mathbf{X} \boldsymbol{\beta})}{2}} \\
& =\frac{1}{\left(2 \pi \sigma^{2}\right)^{n / 2}|\mathbf{D}|^{1 / 2}} \cdot e^{-\frac{(\mathbf{y}-\mathbf{X} \boldsymbol{\beta})^{T} \mathbf{D}^{-1}(\mathbf{y}-\mathbf{X} \boldsymbol{\beta})}{2 \sigma^{2}}}
\end{aligned}
$$

We assign a normal prior distribution to our linear regression coefficients, $\boldsymbol{\beta}$,

$$
\begin{aligned}
\mathrm{p}\left(\boldsymbol{\beta} \mid \sigma^{2}\right) & =\mathcal{N}\left(\boldsymbol{\beta} \mid \mathbf{m}, \sigma^{2} \mathbf{V}\right) \\
& =\frac{1}{(2 \pi)^{p / 2}\left|\sigma^{2} \mathbf{V}\right|^{1 / 2}} e^{-\frac{1}{2}(\boldsymbol{\beta}-\mathbf{m})^{T}\left(\sigma^{2} \mathbf{V}\right)^{-1}(\boldsymbol{\beta}-\mathbf{m})} \\
& =\frac{1}{\left(2 \pi \sigma^{2}\right)^{p / 2}|\mathbf{V}|^{1 / 2}} e^{-\frac{(\boldsymbol{\beta}-\mathbf{m})^{T} \mathbf{V}^{-1}(\boldsymbol{\beta}-\mathbf{m})}{2 \sigma^{2}}}
\end{aligned}
$$

Since $\sigma^{2}$ is a positive quantity, we want to assign it a prior PDF which has support over the positive domain. One such PDF is the inverse-gamma distribution, which is defined by shape parameters $a$ and $d$ :

$$
\begin{aligned}
\mathrm{p}\left(\sigma^{2}\right) & =\mathrm{IG}(a, d) \\
& =\frac{(a / 2)^{d / 2}}{\Gamma(d / 2)}\left(\sigma^{2}\right)^{-(d+2) / 2} e^{-a /\left(2 \sigma^{2}\right)}
\end{aligned}
$$

Thus, we can define our prior distribution as

$$
\begin{aligned}
\mathrm{p}(\boldsymbol{\theta}) & =\mathrm{p}\left(\boldsymbol{\beta}, \sigma^{2}\right) \\
& =\mathrm{p}\left(\boldsymbol{\beta} \mid \sigma^{2}\right) \mathrm{p}\left(\sigma^{2}\right) \\
& =\mathrm{NIG}(a, d, \mathbf{m}, \mathbf{V}) \\
& =\frac{(a / 2)^{d / 2}}{(2 \pi)^{p / 2}|\mathbf{V}|^{1 / 2} \Gamma(d / 2)}\left(\sigma^{2}\right)^{-(d+p+2) / 2} e^{-\frac{(\boldsymbol{\beta}-\mathbf{m})^{T} \mathbf{V}^{-1}(\boldsymbol{\beta}-\mathbf{m})+a}{2 \sigma^{2}}}
\end{aligned}
$$


where NIG denotes the normal-inverse-gamma distribution, i.e., the product of a normal PDF and an inverse-gamma PDF.

By Bayes' theorem, the posterior PDF is

$$
\mathrm{p}(\boldsymbol{\theta} \mid \mathcal{D})=\frac{\mathrm{p}(\mathcal{D} \mid \boldsymbol{\theta}) \mathrm{p}(\boldsymbol{\theta})}{\mathrm{p}(\mathcal{D} \mid \mathcal{M})}
$$

where $\mathrm{p}(\mathcal{D} \mid \mathcal{M})$ is the marginal likelihood.

The posterior distribution is of the form

$$
\begin{aligned}
\mathrm{p}(\boldsymbol{\theta} \mid D)= & \mathrm{p}\left(\boldsymbol{\beta}, \sigma^{2} \mid \mathbf{y}\right) \\
\propto & \mathrm{p}(\mathcal{D} \mid \boldsymbol{\theta}) \mathrm{p}(\boldsymbol{\theta}) \\
= & \frac{1}{\left(2 \pi \sigma^{2}\right)^{n / 2}|\mathbf{D}|^{1 / 2}} \cdot e^{-\frac{(\mathbf{y}-\mathbf{X} \boldsymbol{\beta})^{T} \mathbf{D}^{-1}(\mathbf{y}-\mathbf{X} \boldsymbol{\beta})}{2 \sigma^{2}}} \\
& \times \frac{(a / 2)^{d / 2}}{(2 \pi)^{p / 2}|\mathbf{V}|^{1 / 2} \Gamma(d / 2)}\left(\sigma^{2}\right)^{-(d+p+2) / 2} \cdot e^{-\frac{(\boldsymbol{\beta}-\mathbf{m})^{T} \mathbf{V}^{-1}(\boldsymbol{\beta}-\mathbf{m})+a}{2 \sigma^{2}}} \\
\propto & \left(\sigma^{2}\right)^{-(d+n+p+2) / 2} \cdot e^{-\frac{(\boldsymbol{\beta}-\mathbf{m})^{T} \mathbf{V}^{-1}(\boldsymbol{\beta}-\mathbf{m})+a}{2 \sigma^{2}}} \cdot e^{-\frac{(\mathbf{y}-\mathbf{X} \boldsymbol{\beta})^{T} \mathbf{D}^{-1}(\mathbf{y}-\mathbf{X} \boldsymbol{\beta})}{2 \sigma^{2}}} \\
\propto & \left(\sigma^{2}\right)^{-(d+n+p+2) / 2} \cdot e^{-Q /\left(2 \sigma^{2}\right)}
\end{aligned}
$$

where,

$$
\begin{aligned}
Q= & (\mathbf{y}-\mathbf{X} \boldsymbol{\beta})^{T} \mathbf{D}^{-1}(\mathbf{y}-\mathbf{X} \boldsymbol{\beta})+(\boldsymbol{\beta}-\mathbf{m})^{T} \mathbf{V}^{-1}(\boldsymbol{\beta}-\mathbf{m})+a \\
= & \boldsymbol{\beta}^{T}\left(\mathbf{V}^{-1}+\mathbf{X}^{T} \mathbf{D}^{-1} \mathbf{X}\right) \boldsymbol{\beta}-\boldsymbol{\beta}^{T}\left(\mathbf{V}^{-1} \mathbf{m}+\mathbf{X}^{T} \mathbf{D}^{-1} \mathbf{y}\right) \\
& -\left(\mathbf{m}^{T} \mathbf{V}^{-1}+\mathbf{y}^{T} \mathbf{D}^{-1} \mathbf{X}\right) \boldsymbol{\beta}+\left(\mathbf{m}^{T} \mathbf{V}^{-1} \mathbf{m}+\mathbf{y}^{T} \mathbf{D}^{-1} \mathbf{y}+a\right) \\
= & \left(\boldsymbol{\beta}-\mathbf{m}^{*}\right)^{T}\left(\mathbf{V}^{*}\right)^{-1}\left(\boldsymbol{\beta}-\mathbf{m}^{*}\right)+a^{*}
\end{aligned}
$$

with,

$$
\begin{aligned}
\mathbf{V}^{*} & =\left(\mathbf{V}^{-1}+\mathbf{X}^{T} \mathbf{D}^{-1} \mathbf{X}\right)^{-1} \\
\mathbf{m}^{*} & =\left(\mathbf{V}^{-1}+\mathbf{X}^{T} \mathbf{D}^{-1} \mathbf{X}\right)^{-1}\left(\mathbf{V}^{-1} \mathbf{m}+\mathbf{X}^{T} \mathbf{D}^{-1} \mathbf{y}\right) \\
& =\mathbf{V}^{*}\left(\mathbf{V}^{-1} \mathbf{m}+\mathbf{X}^{T} \mathbf{D}^{-1} \mathbf{y}\right) \\
a^{*} & =a+\mathbf{m}^{T} \mathbf{V}^{-1} \mathbf{m}+\mathbf{y}^{T} \mathbf{D}^{-1} \mathbf{y}-\left(\mathbf{m}^{*}\right)^{T}\left(\mathbf{V}^{*}\right)^{-1} \mathbf{m}^{*} \\
d^{*} & =d+n
\end{aligned}
$$

The results in equation (B8) can be proved by first noting that

$$
\left(\boldsymbol{\beta}-\mathbf{m}^{*}\right)^{T}\left(\mathbf{V}^{*}\right)^{-1}\left(\boldsymbol{\beta}-\mathbf{m}^{*}\right)=\boldsymbol{\beta}^{T} \mathbf{V}^{*^{-1}} \boldsymbol{\beta}-\boldsymbol{\beta}^{T} \mathbf{V}^{*^{-1}} \mathbf{m}^{*}-\mathbf{m}^{*^{T}} \mathbf{V}^{*^{-1}} \boldsymbol{\beta}+\mathbf{m}^{*^{T}} \mathbf{V}^{*^{-1}} \mathbf{m}^{*}
$$

and

$$
\mathbf{V}^{*^{-1}} \mathbf{m}^{*}=\left(\mathbf{V}^{-1} \mathbf{m}+\mathbf{X}^{T} \mathbf{D}^{-1} \mathbf{y}\right)
$$

and

$$
\begin{aligned}
\mathbf{m}^{*^{T}} \mathbf{V}^{*^{-1}} \boldsymbol{\beta} & =\left(\mathbf{V}^{-1} \mathbf{m}+\mathbf{X}^{T} \mathbf{D}^{-1} \mathbf{y}\right)^{T} \mathbf{V}^{*} \cdot \mathbf{V}^{*^{-1}} \cdot \boldsymbol{\beta} \\
& =\left(\mathbf{V}^{-1} \mathbf{m}+\mathbf{X}^{T} \mathbf{D}^{-1} \mathbf{y}\right)^{T} \boldsymbol{\beta} \\
& =\left(\mathbf{m}^{T} \mathbf{V}^{-1}+\mathbf{y}^{T} \mathbf{D}^{-1} \mathbf{X}\right) \boldsymbol{\beta}
\end{aligned}
$$


Then,

$$
\begin{aligned}
Q= & \boldsymbol{\beta}^{T}\left(\mathbf{V}^{-1}+\mathbf{X}^{T} \mathbf{D}^{-1} \mathbf{X}\right) \boldsymbol{\beta}-\boldsymbol{\beta}^{T}\left(\mathbf{V}^{-1} \mathbf{m}+\mathbf{X}^{T} \mathbf{D}^{-1} \mathbf{y}\right) \\
& -\left(\mathbf{m}^{T} \mathbf{V}^{-1}+\mathbf{y}^{T} \mathbf{D}^{-1} \mathbf{X}\right) \boldsymbol{\beta}+\left(\mathbf{m}^{T} \mathbf{V}^{-1} \mathbf{m}+\mathbf{y}^{T} \mathbf{D}^{-1} \mathbf{y}+a\right) \\
= & \boldsymbol{\beta}^{T}\left(\mathbf{V}^{-1}+\mathbf{X}^{T} \mathbf{D}^{-1} \mathbf{X}\right) \boldsymbol{\beta}-\boldsymbol{\beta}^{T}\left(\mathbf{V}^{-1} \mathbf{m}+\mathbf{X}^{T} \mathbf{D}^{-1} \mathbf{y}\right) \\
& -\left(\mathbf{m}^{T} \mathbf{V}^{-1}+\mathbf{y}^{T} \mathbf{D}^{-1} \mathbf{X}\right) \boldsymbol{\beta}+\left(\mathbf{m}^{T} \mathbf{V}^{-1} \mathbf{m}+\mathbf{y}^{T} \mathbf{D}^{-1} \mathbf{y}\right) \\
& +a^{*}-\mathbf{m}^{T} \mathbf{V}^{-1} \mathbf{m}-\mathbf{y}^{T} \mathbf{D}^{-1} \mathbf{y}+\left(\mathbf{m}^{*}\right)^{T}\left(\mathbf{V}^{*}\right)^{-1} \mathbf{m}^{*} \\
= & \boldsymbol{\beta}^{T}\left(\mathbf{V}^{-1}+\mathbf{X}^{T} \mathbf{D}^{-1} \mathbf{X}\right) \boldsymbol{\beta}-\boldsymbol{\beta}^{T}\left(\mathbf{V}^{-1} \mathbf{m}+\mathbf{X}^{T} \mathbf{D}^{-1} \mathbf{y}\right) \\
& -\left(\mathbf{m}^{T} \mathbf{V}^{-1}+\mathbf{y}^{T} \mathbf{D}^{-1} \mathbf{X}\right) \boldsymbol{\beta}+a^{*}+\left(\mathbf{m}^{*}\right)^{T}\left(\mathbf{V}^{*}\right)^{-1} \mathbf{m}^{*} \\
= & \boldsymbol{\beta}^{T} \mathbf{V}^{*-1} \boldsymbol{\beta}-\boldsymbol{\beta}^{T} \mathbf{V}^{*-1} \mathbf{m}^{*}-\mathbf{m}^{*} \mathbf{V}^{*-1} \boldsymbol{\beta}+\left(\mathbf{m}^{*}\right)^{T}\left(\mathbf{V}^{*}\right)^{-1} \mathbf{m}^{*}+a^{*} \\
= & \left(\boldsymbol{\beta}-\mathbf{m}^{*}\right)^{T}\left(\mathbf{V}^{*}\right)^{-1}\left(\boldsymbol{\beta}-\mathbf{m}^{*}\right)+a^{*}
\end{aligned}
$$

Substituting for $Q$ in equation (B7), we find

$$
\begin{aligned}
\mathrm{p}(\boldsymbol{\theta} \mid \mathcal{D}) & \propto\left(\sigma^{2}\right)^{-(d+n+p+2) / 2} \cdot e^{-Q /\left(2 \sigma^{2}\right)} \\
& =\left(\sigma^{2}\right)^{-(d+n+p+2) / 2} \cdot e^{-\frac{\left(\beta-\mathbf{m}^{*}\right)^{T}\left(\mathbf{V}^{*}\right)^{-1}\left(\beta-\mathbf{m}^{*}\right)+a^{*}}{2 \sigma^{2}}}
\end{aligned}
$$

Comparing equation (B17) to equation (B5), it can seen by inspection that the posterior PDF has the same form as the prior PDF. Thus, the normal-inverse-gamma distribution is a conjugate prior and the posterior PDF will also have a NIG distribution

$$
\begin{aligned}
\mathrm{p}(\boldsymbol{\theta} \mid \mathcal{D}) & =\operatorname{NIG}\left(a^{*}, d^{*}, \mathbf{m}^{*}, \mathbf{V}^{*}\right) \\
& =\frac{\left(a^{*} / 2\right)^{d^{*} / 2}}{\left.(2 \pi)^{p / 2} \mathbf{V}^{*}\right|^{1 / 2} \Gamma\left(d^{*} / 2\right)}\left(\sigma^{2}\right)^{-\left(d^{*}+p+2\right) / 2} e^{-\frac{\left(\boldsymbol{\beta}-\mathbf{m}^{*}\right)^{T}\left(\mathbf{V}^{*}\right)-1\left(\boldsymbol{\beta}-\mathbf{m}^{*}\right)+a^{*}}{2 \sigma^{2}}}
\end{aligned}
$$

The marginal likelihood is

$$
\begin{aligned}
& \mathrm{p}(\mathcal{D} \mid \mathcal{M})=\frac{\mathrm{p}(\mathcal{D} \mid \boldsymbol{\theta}) p(\boldsymbol{\theta})}{\mathrm{p}(\boldsymbol{\theta} \mid \mathcal{D})} \\
& =\frac{1}{\left(2 \pi \sigma^{2}\right)^{n / 2}|\mathbf{D}|^{1 / 2}} \cdot e^{-\frac{(\mathbf{y}-\mathbf{X} \mathbf{\beta})^{T} \mathbf{D}^{-1}(\mathbf{y}-\mathbf{X} \mathbf{\beta})}{2 \sigma^{2}}} \\
& \times \frac{(a / 2)^{d / 2}}{(2 \pi)^{p / 2}|\mathbf{V}|^{1 / 2} \Gamma(d / 2)}\left(\sigma^{2}\right)^{-(d+p+2) / 2} e^{-\frac{(\beta-\mathbf{m})^{T} \mathbf{V}^{-1}(\beta-\mathbf{m})+a}{2 \sigma^{2}}} \\
& \div \frac{\left(a^{*} / 2\right)^{d^{*} / 2}}{(2 \pi)^{p / 2}\left|\mathbf{V}^{*}\right|^{1 / 2} \Gamma\left(d^{*} / 2\right)}\left(\sigma^{2}\right)^{-\left(d^{*}+p+2\right) / 2} e^{-\frac{\left(\beta-\mathbf{m}^{*}\right)^{T}\left(\mathbf{V}^{*}-1-1\left(\beta-\mathbf{m}^{*}\right)+a^{*}\right.}{2 \sigma^{2}}} \\
& =\frac{1}{\left(2 \pi \sigma^{2}\right)^{n / 2}|\mathbf{D}|^{1 / 2}} \cdot e^{-\frac{(\boldsymbol{y}-\mathbf{X} \beta)^{T} \mathbf{D}^{-1}(y-\mathbf{X} \beta)}{2 \sigma^{2}}} \frac{(a / 2)^{d / 2}\left|\mathbf{V}^{*}\right|^{1 / 2} \Gamma\left(d^{*} / 2\right)}{\left(a^{*} / 2\right)^{d^{*} / 2}|\mathbf{V}|^{1 / 2} \Gamma(d / 2)}\left(\sigma^{2}\right)^{-\left(d-d^{*}\right) / 2} \\
& \times e^{-\frac{(\beta-\mathbf{m})^{T} \mathbf{V}^{-1}(\boldsymbol{\beta}-\mathbf{m})+a-\left(\boldsymbol{\beta}-\mathbf{m}^{*}\right)^{T}\left(\mathbf{V}^{*}\right)^{-1}\left(\boldsymbol{\beta}-\mathbf{m}^{*}\right)-a^{*}}{2 \sigma^{2}}} \\
& =\frac{1}{\left(2 \pi \sigma^{2}\right)^{n / 2}|\mathbf{D}|^{1 / 2}} \frac{(a / 2)^{d / 2}\left|\mathbf{V}^{*}\right|^{1 / 2} \Gamma\left(d^{*} / 2\right)}{\left(a^{*} / 2\right)^{d^{*} / 2}|\mathbf{V}|^{1 / 2} \Gamma(d / 2)}\left(\sigma^{2}\right)^{-\left(d-d^{*}\right) / 2} \\
& \times e^{-\frac{(\mathbf{y}-\mathbf{X} \beta)^{T} \mathbf{D}^{-1}(\mathbf{y}-\mathbf{X} \boldsymbol{\beta})}{2 \sigma^{2}}} e^{-\frac{(\boldsymbol{\beta}-\mathbf{m})^{T} \mathbf{V}^{-1}(\boldsymbol{\beta}-\mathbf{m})+a}{2 \sigma^{2}}} e^{+Q /\left(2 \sigma^{2}\right)} \\
& =\frac{1}{(2 \pi)^{n / 2}|\mathbf{D}|^{1 / 2}} \frac{(a / 2)^{d / 2}}{\left(a^{*} / 2\right)^{d^{*} / 2}} \frac{\left|\mathbf{V}^{*}\right|^{1 / 2}}{|\mathbf{V}|^{1 / 2}} \frac{\Gamma\left(d^{*} / 2\right)}{\Gamma(d / 2)} \\
& \times e^{-\frac{(\mathbf{y}-\mathbf{X} \boldsymbol{\beta})^{T} \mathbf{D}^{-1}(\mathbf{y}-\mathbf{X} \boldsymbol{\beta})+(\boldsymbol{\beta}-\mathbf{m})^{T} \mathbf{V}^{-1}(\boldsymbol{\beta}-\mathbf{m})+a-\left[(\mathbf{y}-\mathbf{X} \beta)^{T} \mathbf{D}^{-1}(\mathbf{y}-\mathbf{X} \boldsymbol{\beta})+(\boldsymbol{\beta}-\mathbf{m})^{T} \mathbf{V}^{-1}(\boldsymbol{\beta}-\mathbf{m})+a\right]}{2 \sigma^{2}}} \\
& =\frac{1}{(2 \pi)^{n / 2}|\mathbf{D}|^{1 / 2}} \frac{\left|\mathbf{V}^{*}\right|^{1 / 2}}{|\mathbf{V}|^{1 / 2}} \frac{(a / 2)^{d / 2}}{\left(a^{*} / 2\right)^{d^{*} / 2}} \frac{\Gamma\left(d^{*} / 2\right)}{\Gamma(d / 2)}
\end{aligned}
$$

The choice of the data likelihood, $\mathrm{p}(\mathcal{D} \mid \boldsymbol{\theta})=\mathcal{N}\left(\mathcal{D} \mid \boldsymbol{\beta}, \sigma^{2}\right)$, the prior PDF on the slip model, $\mathrm{p}\left(\boldsymbol{\beta} \mid \sigma^{2}\right)=$ $\mathcal{N}\left(\boldsymbol{\beta} \mid \mathbf{m}, \sigma^{2} \mathbf{V}\right)$, and the prior PDF on the regression variance, $\mathrm{p}\left(\sigma^{2}\right)=\mathrm{IG}(a, d)$ are all justifiable on their own. (Gaussian data likelihoods are the most commonly used, and their choice is justified by the principle 
of maximum entropy [see, e.g., Jaynes, 2003; Beck, 2010]. Similarly, Gaussian priors are commonly used for regression variables. Finally, the inverse-gamma distribution satisfies the need for the regression variance to only have support for positive values.) However, it is by choosing these specific families of PDFs for the data likelihood and prior PDFs that we obtain a conjugate prior (i.e., a NIG prior PDF that when multiplied by the normally distributed data likelihood yields a NIG posterior PDF), and because we have used a conjugate prior, we obtain an analytical expression for the marginal likelihood, $\mathrm{p}(\mathcal{D} \mid \mathcal{M})$.

In many cases, the regression variance, $\sigma^{2}$, is a nuisance parameter, and we only wish to make posterior inference on $\boldsymbol{\beta}$, so that we desire $\mathrm{p}(\boldsymbol{\beta} \mid \mathcal{D})$ and not $\mathrm{p}\left(\boldsymbol{\beta}, \sigma^{2} \mid \mathcal{D}\right)$. To obtain the mean and variance of the marginal PDF, $\mathrm{p}(\boldsymbol{\beta} \mid \mathcal{D})$, we first need to derive the mean and variance of $\mathrm{p}(\boldsymbol{\beta})$ and $\mathrm{p}\left(\sigma^{2}\right)$. From the definition of the mean and variance of the inverse-gamma distribution

$$
\begin{gathered}
E\left(\sigma^{2}\right)=\frac{a}{d-2} \quad \text { for } d>2 \\
\operatorname{var}\left(\sigma^{2}\right)=\frac{2 a^{2}}{(d-2)^{2}(d-4)} \quad \text { for } d>4
\end{gathered}
$$

The prior on $\boldsymbol{\beta}$ conditional on $\sigma^{2}$ is $\mathcal{N}\left(\mathbf{m}, \sigma^{2} \mathbf{V}\right)$. Thus,

$$
\begin{gathered}
E\left(\boldsymbol{\beta} \mid \sigma^{2}\right)=\mathbf{m} \\
\operatorname{var}\left(\boldsymbol{\beta} \mid \sigma^{2}\right)=\sigma^{2} \mathbf{V}
\end{gathered}
$$

Recalling the law of total expectation, $E(\mathbf{Y})=E(E(\mathbf{Y} \mid \mathbf{X}))$, and the law of total variance, $\operatorname{var}(\mathbf{Y})=E(\operatorname{var}(\mathbf{Y} \mid \mathbf{X}))+\operatorname{var}(E(\mathbf{Y} \mid \mathbf{X}))$

$$
\begin{aligned}
& E(\boldsymbol{\beta})=E\left(E\left(\boldsymbol{\beta} \mid \sigma^{2}\right)\right)=\mathbf{m} \\
\operatorname{var}(\boldsymbol{\beta}) & =E\left(\operatorname{var}\left(\boldsymbol{\beta} \mid \sigma^{2}\right)\right)+\operatorname{var}\left(E\left(\boldsymbol{\beta} \mid \sigma^{2}\right)\right) \\
& =E\left(\sigma^{2} \mathbf{V}\right)+\operatorname{var}(\mathbf{m}) \\
& =E\left(\sigma^{2}\right) \mathbf{V} \\
& =\frac{a}{d-2} \mathbf{V}
\end{aligned}
$$

Since this is a conjugate prior, the posterior PDFs will be

$$
\begin{aligned}
\mathrm{p}\left(\sigma^{2} \mid \mathcal{D}\right) & =\operatorname{IG}\left(a^{*}, d^{*}\right) \\
\mathrm{p}\left(\boldsymbol{\beta} \mid \sigma^{2}, \mathcal{D}\right) & =\mathcal{N}\left(\mathbf{m}^{*}, \sigma^{2} \mathbf{V}^{*}\right)
\end{aligned}
$$

Accordingly,

$$
\begin{aligned}
E\left(\sigma^{2} \mid \mathcal{D}\right) & =\frac{a^{*}}{d^{*}-2} \\
\operatorname{var}\left(\sigma^{2} \mid \mathcal{D}\right) & =\frac{2 a^{* 2}}{\left(d^{*}-2\right)^{2}\left(d^{*}-4\right)} \\
E\left(\boldsymbol{\beta} \mid \sigma^{2}, \mathcal{D}\right) & =\mathbf{m}^{*} \\
\operatorname{var}\left(\boldsymbol{\beta} \mid \sigma^{2}, \mathcal{D}\right) & =\sigma^{2} \mathbf{V}^{*} \\
E(\boldsymbol{\beta} \mid \mathcal{D}) & =\mathbf{m}^{*} \\
\operatorname{var}(\boldsymbol{\beta} \mid \mathcal{D}) & =\frac{a^{*}}{d^{*}-2} \mathbf{V}^{*}
\end{aligned}
$$

Note that since $a$ and $d$ are positive and $d^{*}=d+n$ where $n$ is the number of data points, the posterior means and variances $E\left(\sigma^{2} \mid \mathcal{D}\right), \operatorname{var}\left(\sigma^{2} \mid \mathcal{D}\right)$, and $\operatorname{var}(\boldsymbol{\beta} \mid \mathcal{D})$ are guaranteed to exist if there are at least 2,4 , and 2 observations, respectively. 
An analytical expression for the marginal posterior distribution of $\boldsymbol{\beta}, \mathrm{p}(\boldsymbol{\beta} \mid \mathcal{D})$, can be found by integrating the joint posterior with respect to $\sigma^{2}$ :

$$
\begin{aligned}
\mathrm{p}(\boldsymbol{\beta} \mid \mathcal{D}) & =\int_{\sigma^{2}} \mathrm{p}\left(\boldsymbol{\beta}, \sigma^{2} \mid \mathcal{D}\right) \mathrm{d} \sigma^{2} \\
& =\int_{0}^{\infty} \mathrm{NIG}\left(a^{*}, d^{*}, \mathbf{m}^{*}, \mathbf{V}^{*}\right) \mathrm{d} \sigma^{2} \\
& =\int_{0}^{\infty} \frac{\left(a^{*} / 2\right)^{d^{*} / 2}}{(2 \pi)^{p / 2}\left|\mathbf{V}^{*}\right|^{1 / 2} \Gamma\left(d^{*} / 2\right)}\left(\sigma^{2}\right)^{-\left(d^{*}+p+2\right) / 2} e^{-\frac{\left(\boldsymbol{\beta}-\mathbf{m}^{*}\right)^{T}\left(\mathbf{(}^{*}\right)^{-1}\left(\beta-\mathbf{m}^{*}\right)+a^{*}}{2 \sigma^{2}}} \mathrm{~d} \sigma^{2} \\
& =c \int_{0}^{\infty}\left(\sigma^{2}\right)^{-\left(d^{*}+p+2\right) / 2} e^{-\frac{b}{2 \sigma^{2}}} \mathrm{~d} \sigma^{2}
\end{aligned}
$$

with $c=\frac{\left(a^{*} / 2\right)^{d^{*} / 2}}{(2 \pi)^{p / 2}\left|\mathbf{V}^{*}\right|^{1 / 2} \Gamma\left(d^{*} / 2\right)}$ and $b=\left(\boldsymbol{\beta}-\mathbf{m}^{*}\right)^{T}\left(\mathbf{V}^{*}\right)^{-1}\left(\boldsymbol{\beta}-\mathbf{m}^{*}\right)+a^{*}$.

If we make the substitution $z=\frac{b}{2 \sigma^{2}}$ so that $\mathrm{d} \sigma^{2}=-\frac{b}{2 z^{2}} \mathrm{~d} z$, then equation (B34) becomes

$$
\begin{aligned}
\mathrm{p}(\boldsymbol{\beta} \mid \mathcal{D}) & =c \int_{0}^{\infty}\left(\sigma^{2}\right)^{-\left(d^{*}+p+2\right) / 2} e^{-\frac{b}{2 \sigma^{2}}} \mathrm{~d} \sigma^{2} \\
& =c \int_{0}^{\infty}\left(\frac{b}{2 z}\right)^{-\left(d^{*}+p+2\right) / 2} e^{-z} \frac{b}{2 z^{2}} \mathrm{~d} z \\
& =c\left(\frac{b}{2}\right)^{-\left(d^{*}+p\right) / 2}\left[\int_{0}^{\infty}(z)^{\left(d^{*}+p-2\right) / 2} e^{-z} \mathrm{~d} z\right]
\end{aligned}
$$

We can recognize the part of equation (B35) in square brackets as the gamma integral. Thus,

$$
\begin{aligned}
\mathrm{p}(\boldsymbol{\beta} \mid \mathcal{D}) & =c\left(\frac{b}{2}\right)^{-\left(d^{*}+p\right) / 2}\left[\frac{\Gamma\left(\frac{d^{*}+p}{2}\right)}{1}\right] \\
& =\frac{\left(a^{*} / 2\right)^{d^{*} / 2}}{(2 \pi)^{p / 2}\left|\mathbf{V}^{*}\right|^{1 / 2} \Gamma\left(d^{*} / 2\right)} \times\left(\frac{\left(\boldsymbol{\beta}-\mathbf{m}^{*}\right)^{T}\left(\mathbf{V}^{*}\right)^{-1}\left(\boldsymbol{\beta}-\mathbf{m}^{*}\right)+a^{*}}{2}\right)^{-\left(d^{*}+p\right) / 2} \Gamma\left(\frac{d^{*}+p}{2}\right) \\
& =\frac{\left(a^{*}\right)^{d^{*} / 2} \Gamma\left(\left(d^{*}+p\right) / 2\right)}{\left|\mathbf{V}^{*}\right|^{1 / 2} \pi^{p / 2} \Gamma\left(d^{*} / 2\right)} \times\left(\left(\boldsymbol{\beta}-\mathbf{m}^{*}\right)^{T}\left(\mathbf{V}^{*}\right)^{-1}\left(\boldsymbol{\beta}-\mathbf{m}^{*}\right)+a^{*}\right)^{-\left(d^{*}+p\right) / 2} \\
& =\frac{\Gamma\left(\left(d^{*}+p\right) / 2\right)}{\left(a^{*}\right)^{p / 2}\left|\mathbf{V}^{*}\right|^{1 / 2} \pi^{p / 2} \Gamma\left(d^{*} / 2\right)} \times\left(1+\left(\boldsymbol{\beta}-\mathbf{m}^{*}\right)^{T}\left(a^{*} \mathbf{V}^{*}\right)^{-1}\left(\boldsymbol{\beta}-\mathbf{m}^{*}\right)\right)^{-\left(d^{*}+p\right) / 2} \\
& =\frac{\Gamma\left(\left(d^{*}+p\right) / 2\right)}{\left(d^{*}\right)^{p / 2}\left|\frac{a^{*}}{d^{*}} \mathbf{V}^{*}\right|^{1 / 2} \pi^{p / 2} \Gamma\left(d^{*} / 2\right)} \times\left(1+\frac{1}{d^{*}}\left(\boldsymbol{\beta}-\mathbf{m}^{*}\right)^{T}\left(\frac{a^{*}}{d^{*}} \mathbf{V}^{*}\right)^{-1}\left(\boldsymbol{\beta}-\mathbf{m}^{*}\right)\right)^{-\left(d^{*}+p\right) / 2}
\end{aligned}
$$

Equation (B36) is $t_{d^{*}}\left(\mathbf{m}^{*}, \frac{a^{*}}{d^{*}} \mathbf{V}^{*}\right)$, the multivariate $t$ distribution for $d^{*}$ degrees of freedom with mean $\mathbf{m}^{*}$ and scale matrix $\frac{a^{*}}{d^{*}} \mathbf{V}^{*}$.

\section{Notation}

$\mathbf{C}_{\mathrm{d}} \quad n \times n$ covariance matrix of observational errors.

$\mathbf{C}_{\mathbf{p}} n \times n$ covariance matrix of prediction errors.

D $n \times n$ covariance matrix of total errors.

$D$ Vector of data predicted by stochastic forward model.

$E(\cdot) \quad$ Expectation of $(\cdot)$.

$\mathrm{IG}(a, d)$ Inverse-gamma distribution with shape parameters $a$ and $d$ (equation (B4)).

$\mathcal{M}$ Model class.

m $p \times 1$ mean vector for prior PDF on linear regression variables, $\mathrm{p}(\boldsymbol{\beta})=\mathcal{N}\left(\mathbf{m}, \sigma^{2} \mathbf{V}\right)$.

$\mathbf{m}^{*} \quad p \times 1$ Mean vector for posterior PDF on linear regression variables, $\mathrm{p}\left(\boldsymbol{\beta} \mid \sigma^{2}, \mathcal{D}\right)=\mathcal{N}\left(\mathbf{m}^{*}, \sigma^{2} \mathbf{V}^{*}\right)$.

$n$ Number of observed data.

$\mathcal{N}(\mathbf{m}, \mathbf{V}) \quad$ Normal distribution with mean, $\mathbf{m}$, and covariance, $\mathbf{V}$.

$\operatorname{NIG}(a, d, \mathbf{m}, \mathbf{V}) \quad$ Normal-inverse-gamma distribution with parameters $a, d, \mathbf{m}$, and $\mathbf{V}$ (equation (B5)). 


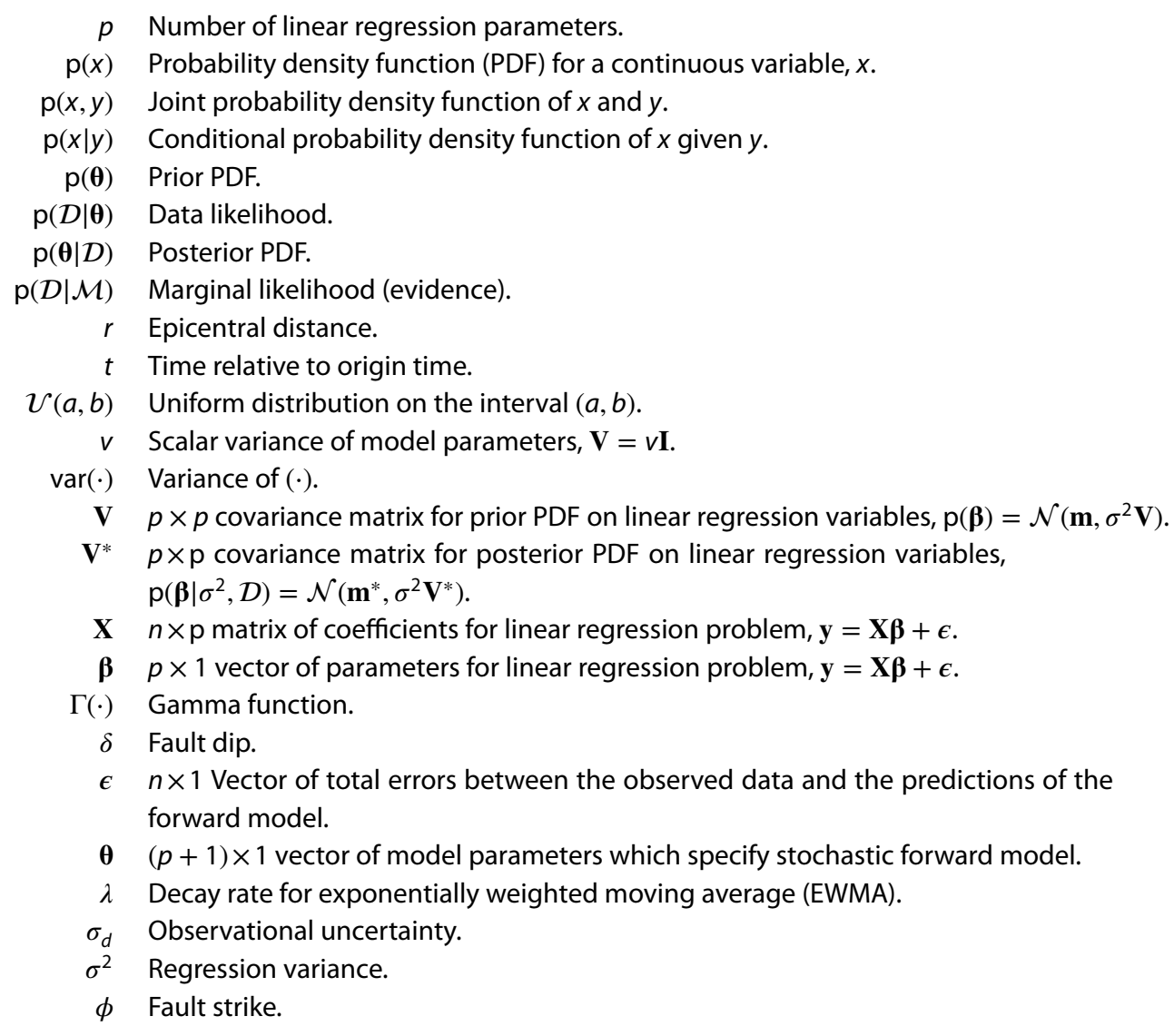

Acknowledgments

The authors wish to thank James Beck, Benjamin Brooks, and Elizabeth Cochran for helpful reviews. Brad Aagaard provided the time histories of surface displacement for the Hayward fault scenario earthquake. Yehuda Bock and Brendan Crowell provided $1 \mathrm{~Hz}$ GPS solutions, and Hiroo Kanamori provided the source time function for the 2003 Tokachi-oki, Japan earthquake. Asaf Inbal and Susan Owen provided the $1 \mathrm{~Hz}$ GPS solutions for the 2011 Tohoku-oki, Japan earthquake.

\section{References}

Aagaard, B. T., R. W. Graves, D. P. Schwartz, D. A. Ponce, and R. W. Graymer (2010), Ground-motion modeling of Hayward fault scenario earthquakes, part I: Construction of the suite of scenarios, Bull. Seismol. Soc. Am., 100(6), 2927-2944, doi:10.1785/0120090324.

Allen, R. M., and A. Ziv (2011), Application of real-time GPS to earthquake early warning, Geophys. Res. Lett., 38, L16310, doi:10.1029/2011GL047947.

Allen, R. V. (1978), Automatic earthquake recognition and timing from single traces, Bull. Seismol. Soc. Assoc., 68(5), $1521-1532$.

Bayes, T. (1763), An essay towards solving a problem in the doctrine of chances. By the late Rev. Mr. Bayes, F. R. S. communicated by Mr. Price, in a letter to John Canton, A. M. F. R. S., Philos. T. R. Soc., 53, 370-418.

Beck, J. L. (2010), Bayesian system identification based on probability logic, Struct. Control Health Monit., 17, 825-847, doi:10.1002/stc.424. Bellman, R. (1957), Dynamic Programming, Princeton Univ. Press, Princeton, N. J., U.S.A.

Chib, S., and E. Greenberg (1995), Understanding the Metropolis-Hastings algorithm, Amer. Stat., 49(4), 327-335.

Crowell, B. W., Y. Bock, and M. B. Squibb (2009), Demonstration of earthquake early warning using total displacement waveforms from real-time GPS networks, Seismol. Res. Lett., 80, 772-782, doi:10.1785/gssrl.80.5.772.

Crowell, B. W., Y. Bock, and D. Melgar (2012), Real-time inversion of GPS data for finite fault modeling and rapid hazard assessment, Geophys. Res. Lett., 39, L09305, doi:10.1029/2012GL051318.

Duputel, Z., L. Rivera, H. Kanamori, G. P. Hayes, B. Hirshorn, and S. Weinstein (2011), Real-time W phase inversion during the 2011 off the Pacific coast of Tohoku earthquake, Earth Planet Space, 63(7), 535-539, doi:10.5047/eps.2011.05.032.

Fukuda, J., and K. M. Johnson (2008), A fully Bayesian inversion for spatial distribution of fault slip with objective smoothing, Bull. Seismol. Soc. Am., 98(3), 1128-1146, doi:10.1785/0120070194.

Fukuda, J., and K. M. Johnson (2010), Mixed linear-non-linear inversion of crustal deformation data: Bayesian inference of model, weighting and regularization parameters, Geophys. J. Int., 181(3), 1441-1458, doi:10.1111/j.1365-246X.2010.04564.x.

Jaynes, E. T. (2003), Probability Theory: The Logic of Science, G. L. Bretthorst (Ed.), Cambridge Univ. Press, Cambridge, U. K.

Langbein, J., and Y. Bock (2004), High-rate real-time GPS network at Parkfield: Utility for detecting fault slip and seismic displacements, Geophys. Res. Lett., 31, L15S20, doi:10.1029/2003GL019408.

Melgar, D., Y. Bock, and B. W. Crowell (2012), Real-time centroid moment tensor determination for large earthquakes from local and regional displacement records, Geophys. J. Int., 188, 703-718, doi:10.1111/j.1365-246X.2011.05297.x.

Metropolis, N., A. Rosenbluth, M. Rosenbluth, A. Teller, and E. Teller (1953), Equation of state calculations by fast computing machines, J. Chem. Phys., 21(6), 1087-1092.

Minson, S. E., M. Simons, and J. L. Beck (2013), Bayesian inversion for finite fault earthquake source models I: Theory and algorithm, Geophys. J. Int., 194(3), 1701-1726, doi:10.1093/gji/ggt180.

Monelli, D., and P. M. Mai (2008), Bayesian inference of kinematic earthquake rupture parameters through fitting of strong motion data, Geophys. J. Int., 173, 220-232, doi:10.1111/j.1365-246X.2008.03733.x.

Mosegaard, K., and A. Tarantola (1995), Monte Carlo sampling of solutions to inverse problems, J. Geophys. Res., 100(B7), 12,431-12,447.

Muto, M., and J. L. Beck (2008), Bayesian updating and model class selection for hysteretic structural models using stochastic simulation, J. Vib. Control, 14(1-2), 7-34, doi:10.1177/1077546307079400. 
O'Hagan, A. (1994), Kendall's Advanced Theory of Statistics, Vol. 2b: Bayesian Statistics, John Wiley, London.

Ohta, Y., et al. (2012), Quasi real-time fault model estimation for near-field tsunami forecasting based on RTK-GPS analysis: Application to the 2011 Tohoku-Oki earthquake (Mw 9.0), J. Geophys. Res., 117, B02311, doi:10.1029/2011JB008750.

Okada, Y. (1985), Surface deformation due to shear and tensile faults in a half-space, Bull. Seismol. Soc. Am., 75(4), 1135-1154.

Roberts, S. V. (1959), Control chart tests based on geometric moving averages, Technometrics, 1, 239-250.

Wells, D. L., and K. J. Coppersmith (1994), New empirical relationships among magnitude, rupture length, rupture width, rupture area, and surface displacement, Bull. Seismol. Soc. Am., 84(4), 974-1002.

Wright, T. J., N. Houlie, M. Hildyard, and T. Iwabuchi (2012), Real-time, reliable magnitudes for large earthquakes from $1 \mathrm{~Hz}$ GPS precise point positioning: The 2011 Tohoku-Oki (Japan) earthquake, Geophys. Res. Lett., 39, L12302, doi:10.1029/2012GL051894.

Wu, Y.-M., and L. Zhao (2006), Magnitude estimation using the first three seconds P-wave amplitude in earthquake early warning, Geophys. Res. Lett., 33, L16312, doi:10.1029/2006GL026871. 
Auxiliary Material for

Real-time inversions for finite fault slip models and rupture geometry based on high-rate GPS data.

$$
\begin{gathered}
\text { Sarah E. Minson }{ }^{1} \text {, Jessica R. Murray }{ }^{2} \text {, John O. Langbein², and Joan S. Gomberg }{ }^{1} \\
\left({ }^{1}\right. \text { USGS Earthquake Science Center, Seattle, Washington, USA, } \\
{ }^{2} \text { USGS Earthquake Science Center, Menlo Park, California, USA.) } \\
\text { Journal of Geophysical Research, Solid Earth, } 2013
\end{gathered}
$$

Introduction

This supplementary material contains data set contains movies depicting the finite fault slip model for each of the three test earthquakes (2011 Tohoku-oki, 2003 Tokahi-oki, Hayward fault scenario) using each of three different data processing schemes (raw data, data smoothed with an exponentially weighted moving average (EWMA) with a decay constant of 0.1 , and data smoothed with an EWMA with a decay constant of 0.01). The first supplementary figure illustrates the sensitivity of the inversion to the choice of a priori covariance on the slip parameters. Additional supplementary figures show the inversion results that were animated in the movies in fault-view so that the distribution of slip on the fault may be more clearly seen. The final nine supplemental figures are the same as Supplemental figures 5-13 are the same as Figures 13-21 in the main paper except the black and red vectors show the observed and predicted vertical surface displacements and the gray vectors show the slip direction on each patch with length scaled by the slip on that patch.

1. ms01.avi (48MB AVI file) Inversion of Tohoku-oki $1 \mathrm{~Hz}$ GPS data using offsets estimated from raw time series.

2. ms02.avi (44MB AVI file) Inversion of Tohoku-oki $1 \mathrm{~Hz}$ GPS data using offsets estimated from time series smoothed using an exponentially weighted moving average (EWMA) with a decay constant of 0.1 .

3. ms03.avi (39MB AVI file) Inversion of Tohoku-oki $1 \mathrm{~Hz}$ GPS data using offsets estimated from time series smoothed using an EWMA with a decay constant of 0.01 .

4. ms04.avi (24MB AVI file) Inversion of Tokachi-oki $1 \mathrm{~Hz}$ GPS data using offsets estimated from raw time series.

5. ms05.avi (23MB AVI file) Inversion of Tokachi-oki $1 \mathrm{~Hz}$ GPS data using offsets estimated from time series smoothed using an EWMA with a decay constant of 0.1.

6. ms06.avi (21MB AVI file) Inversion of Tokachi-oki $1 \mathrm{~Hz}$ GPS data using offsets estimated from time series smoothed using an EWMA with a decay constant of 0.01 .

7. ms07.avi (13MB AVI file) Inversion of Hayward fault simulated $1 \mathrm{~Hz}$ GPS data using offsets estimated from raw time series.

8. ms08.avi (12MB AVI file) Inversion of Hayward fault simulated $1 \mathrm{~Hz}$ GPS data using offsets estimated from time series smoothed using an EWMA with a decay constant of 0.1.

9. ms09.avi (11MB AVI file) Inversion of Hayward fault simulated $1 \mathrm{~Hz}$ GPS data using offsets 
estimated from time series smoothed using an EWMA with a decay constant of 0.01 .

10. fs01.pdf (Supplementary figure 1) Effects on inversion results for slip of varying V. The mean posterior estimate for the strike-slip (left) and dip-slip (right) components of motion are shown for a simulated Mw 9 Tohoku-oki-type earthquake. For reference, the input slip model is shown in the top row.

11. fs02.pdf (Supplementary figure 2) Detail of Tohoku-oki earthquake slip models plotted in Figures $13-15$.

12. fs03.pdf (Supplementary figure 3) Detail of Tokachi-oki earthquake slip models plotted in Figures 16-18.

13. fs04.pdf (Supplementary figure 4) Detail of Hayward fault simulation slip models plotted in Figures 19-21.

14. fs05.pdf (Supplementary figure 5) Result of semi-analytical Bayesian real-time finite fault inversion for the Tohoku-oki earthquake at 60 seconds after the origin time. Same as Figure 13 except black and red vectors show observed and predicted GPS displacements, respectively, and gray vectors are direction of slip on each patch scaled by slip magnitude.

15. fs06.pdf (Supplementary figure 6) Result of semi-analytical Bayesian real-time finite fault inversion for the Tohoku-oki earthquake at 120 seconds after the origin time. Same as Figure 14 except black and red vectors show observed and predicted GPS displacements, respectively, and gray vectors are direction of slip on each patch scaled by slip magnitude.

16. fs07.pdf (Supplementary figure 7) Result of semi-analytical Bayesian real-time finite fault inversion for the Tohoku-oki earthquake at 180 seconds after the origin time. Same as Figure 15 except black and red vectors show observed and predicted GPS displacements, respectively, and gray vectors are direction of slip on each patch scaled by slip magnitude.

17. fs08.pdf (Supplementary figure 8) Result of semi-analytical Bayesian real-time finite fault inversion for the Tokachi-oki earthquake at 30 seconds after the origin time. Same as Figure 16 except black and red vectors show observed and predicted GPS displacements, respectively, and gray vectors are direction of slip on each patch scaled by slip magnitude.

18. fs09.pdf (Supplementary figure 9) Result of semi-analytical Bayesian real-time finite fault inversion for the Tokachi-oki earthquake at 60 seconds after the origin time. Same as Figure 17 except black and red vectors show observed and predicted GPS displacements, respectively, and gray vectors are direction of slip on each patch scaled by slip magnitude.

19. fs 10.pdf (Supplementary figure 10) Result of semi-analytical Bayesian real-time finite fault inversion for the Tokachi-oki earthquake at 90 seconds after the origin time. Same as Figure 18 except black and red vectors show observed and predicted GPS displacements, respectively, and a gray reference vector shows the direction of slip on the patch with maximum slip.

20. fs11.pdf (Supplementary figure 11) Result of semi-analytical Bayesian real-time finite fault inversion for the Hayward fault simulation at 20 seconds after the origin time. Same as Figure 19 except black and red vectors show observed and predicted GPS displacements, 
respectively, and gray vectors are direction of slip on each patch scaled by slip magnitude.

21. fs12.pdf (Supplementary figure 12) Result of semi-analytical Bayesian real-time finite fault inversion for the Hayward fault simulation at 40 seconds after the origin time. Same as Figure 20 except black and red vectors show observed and predicted GPS displacements, respectively, and a gray reference vector shows the direction of slip on the patch with maximum slip.

22. fs13.pdf (Supplementary figure 13) Result of semi-analytical Bayesian real-time finite fault inversion for the Hayward fault simulation at 60 seconds after the origin time. Same as Figure 21 except black and red vectors show observed and predicted GPS displacements, respectively, and a gray reference vector shows the direction of slip on the patch with maximum slip. 


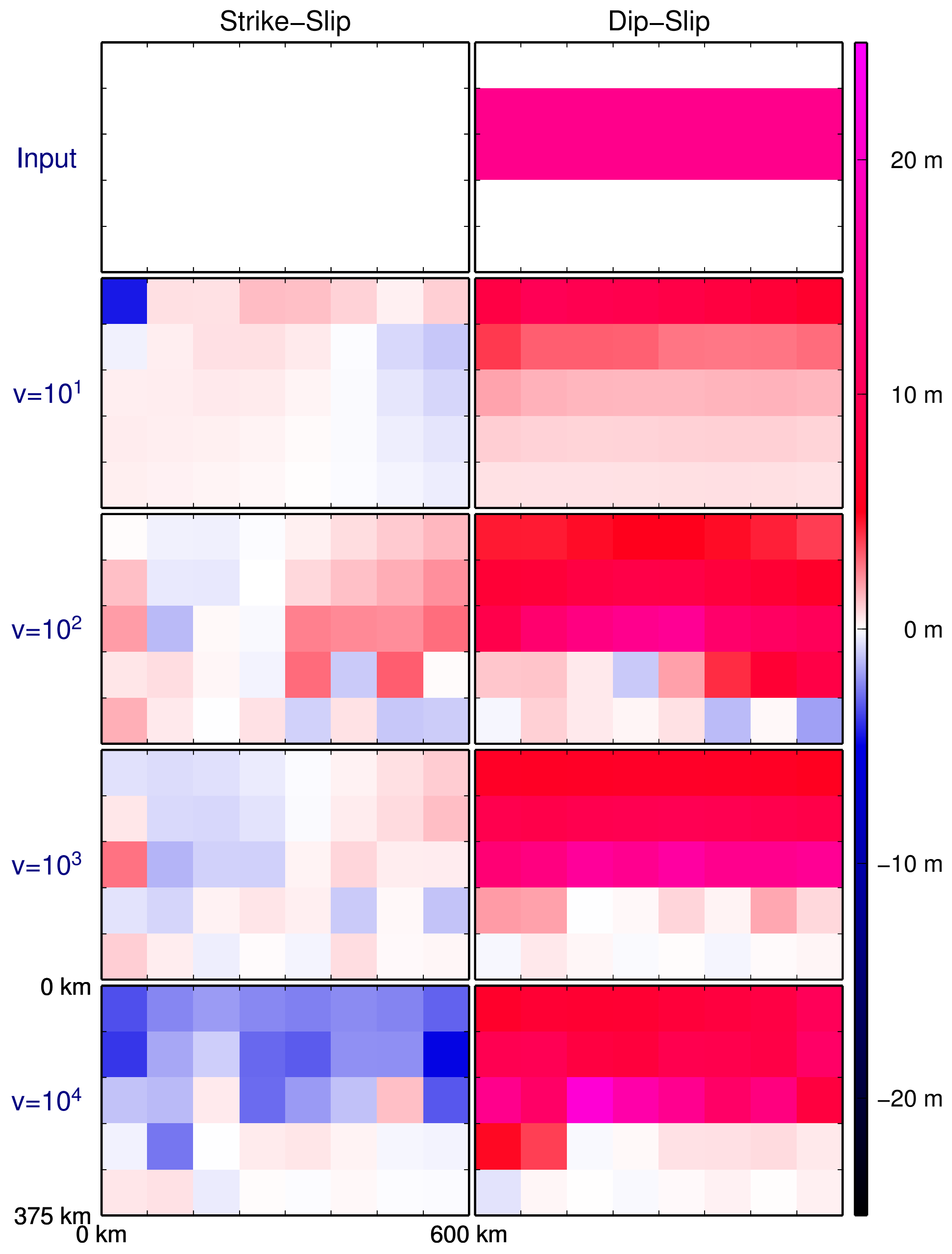




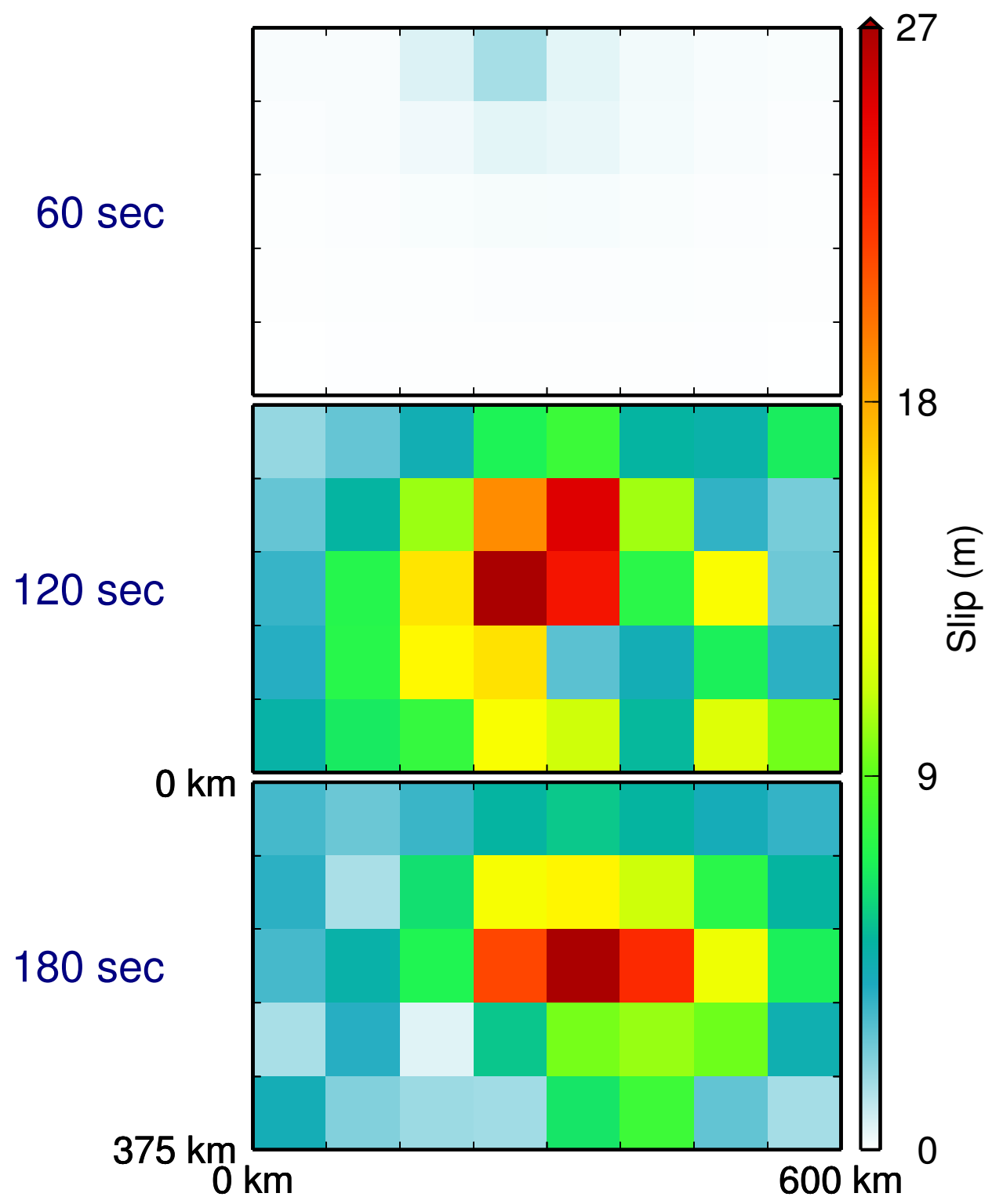




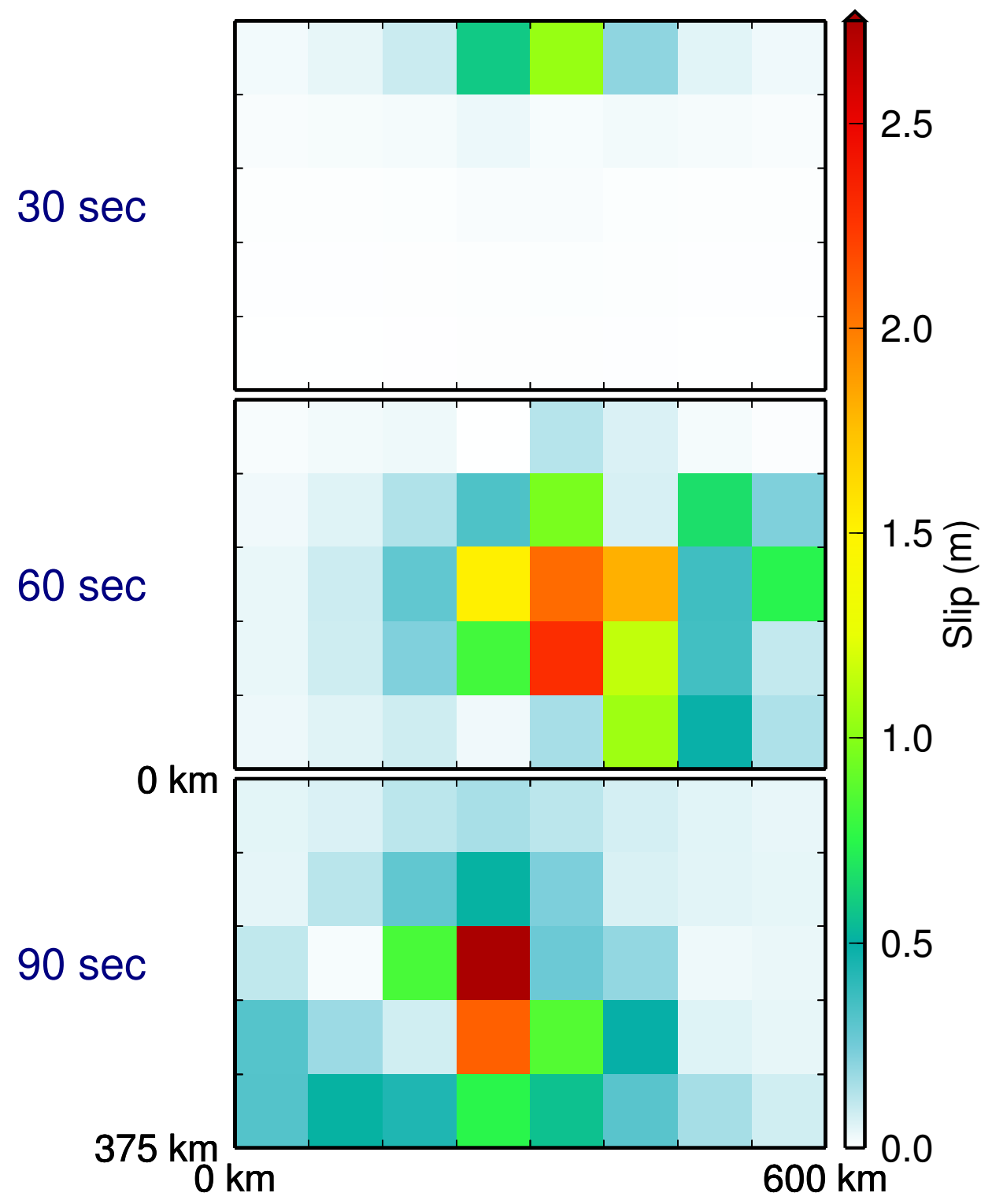




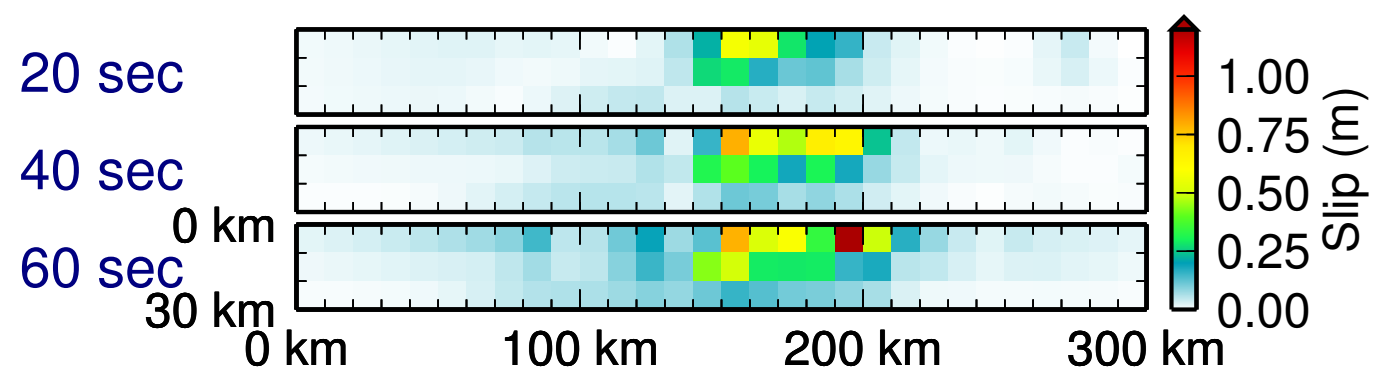


$34^{\circ} \mathrm{N}$

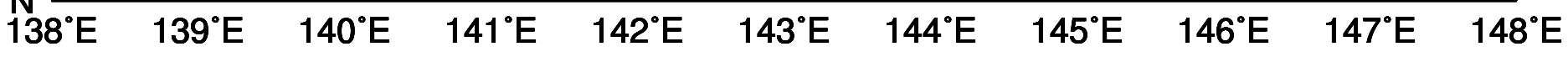

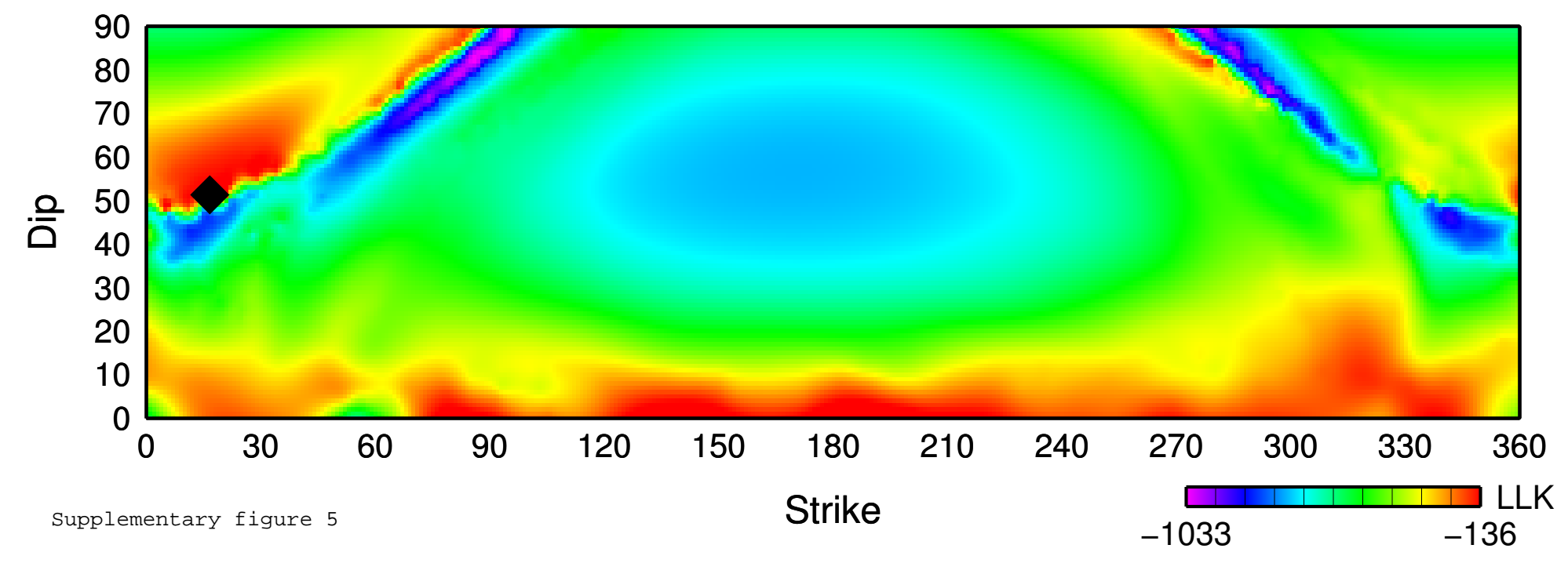



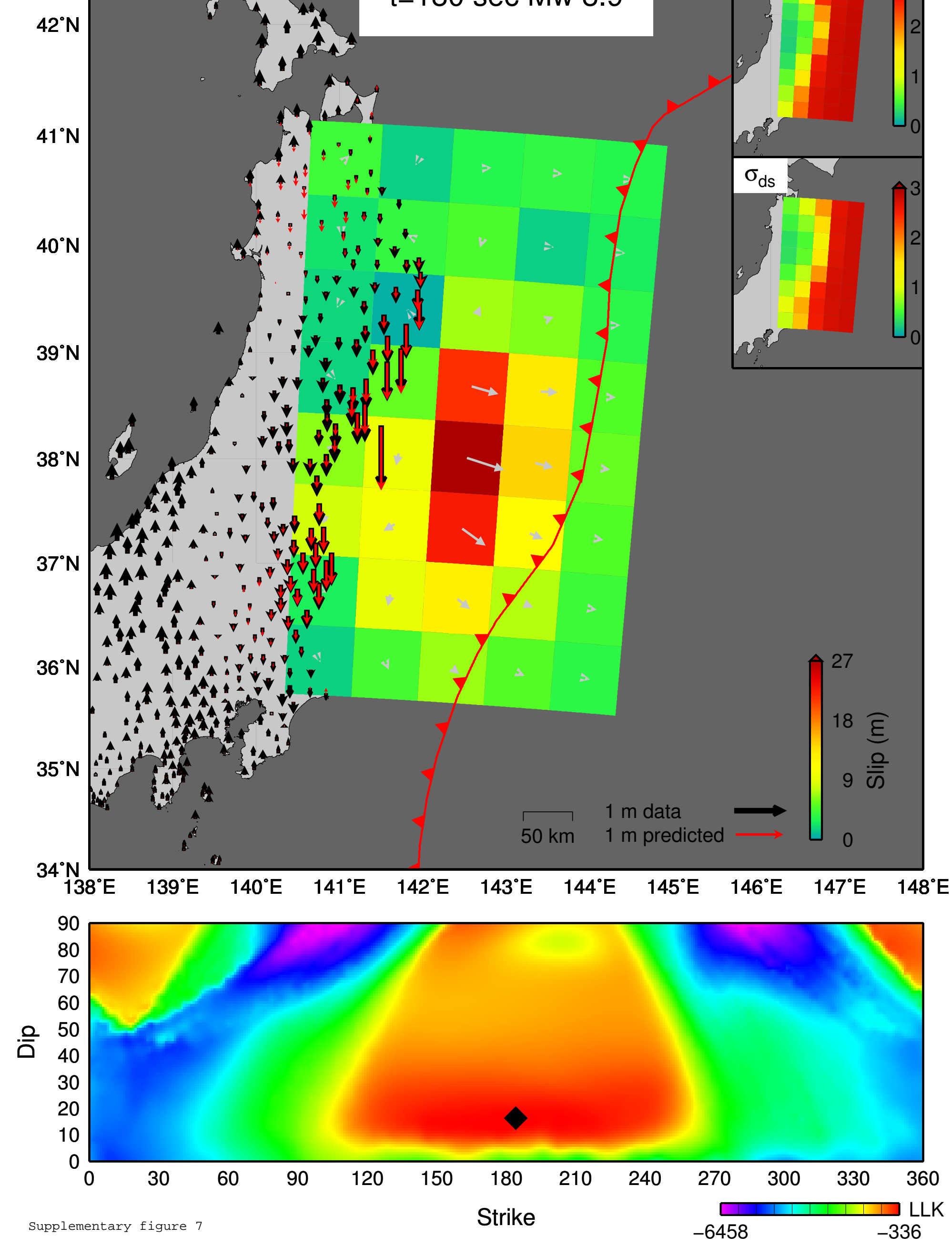


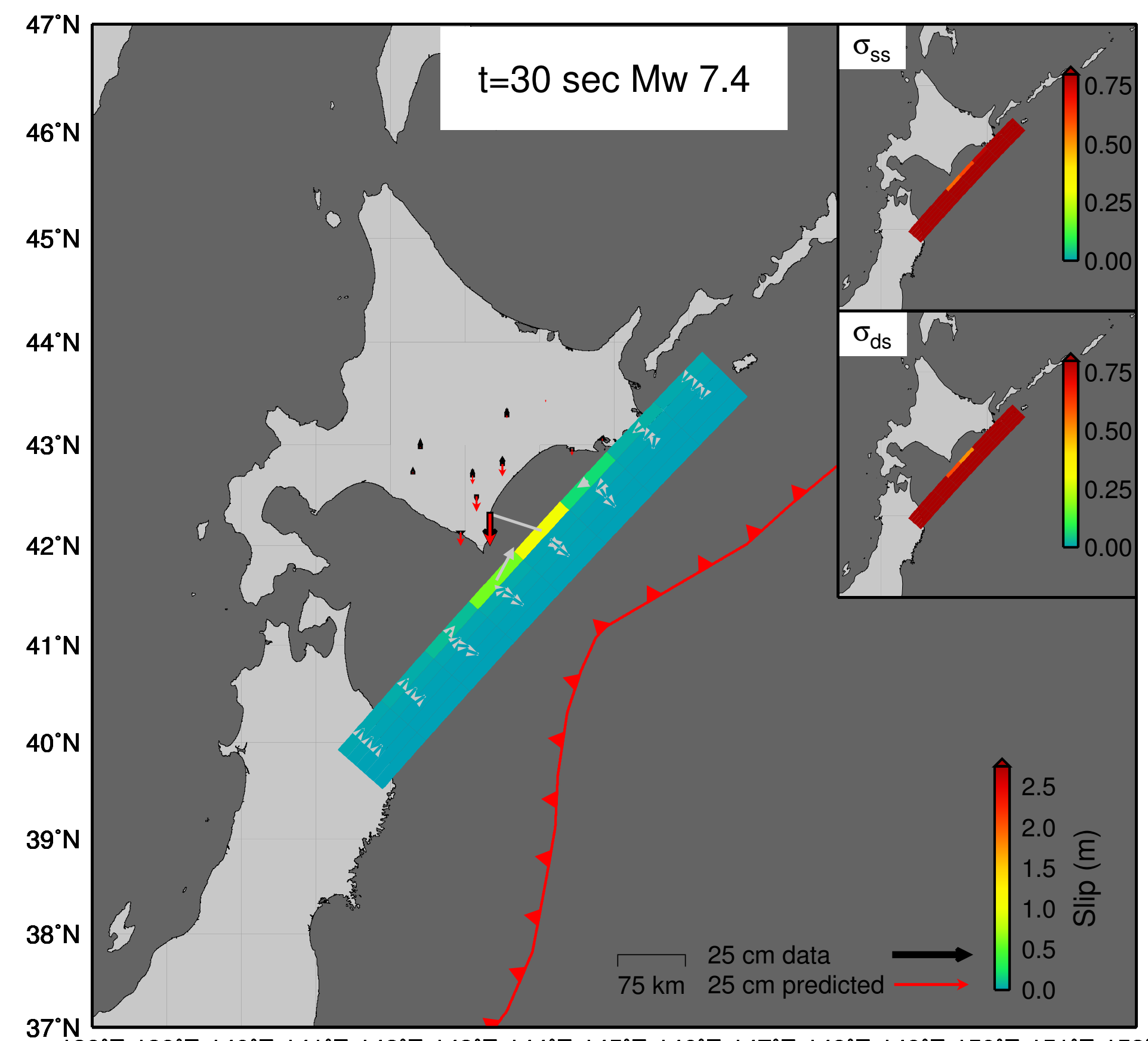

$138^{\circ} \mathrm{E} 139^{\circ} \mathrm{E} 140^{\circ} \mathrm{E} 141^{\circ} \mathrm{E} 142^{\circ} \mathrm{E} 143^{\circ} \mathrm{E} 144^{\circ} \mathrm{E} 145^{\circ} \mathrm{E} 146^{\circ} \mathrm{E} 147^{\circ} \mathrm{E} 148^{\circ} \mathrm{E} 149^{\circ} \mathrm{E} 150^{\circ} \mathrm{E} 151^{\circ} \mathrm{E} 152^{\circ} \mathrm{E}$

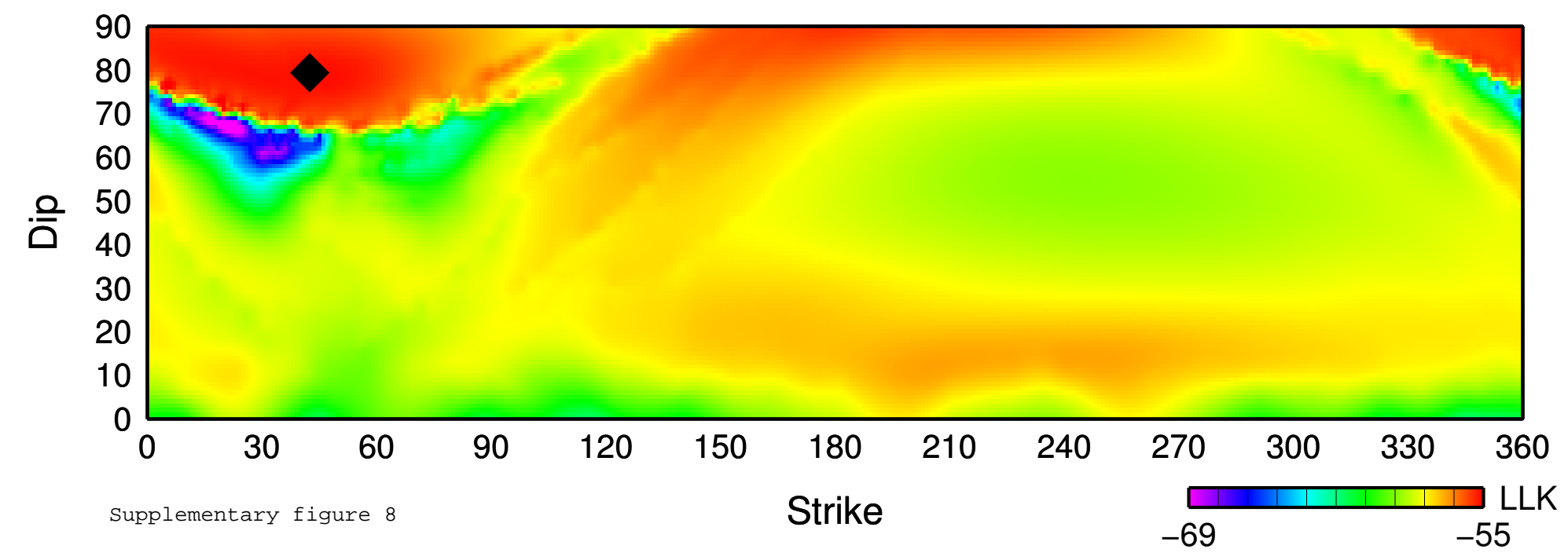




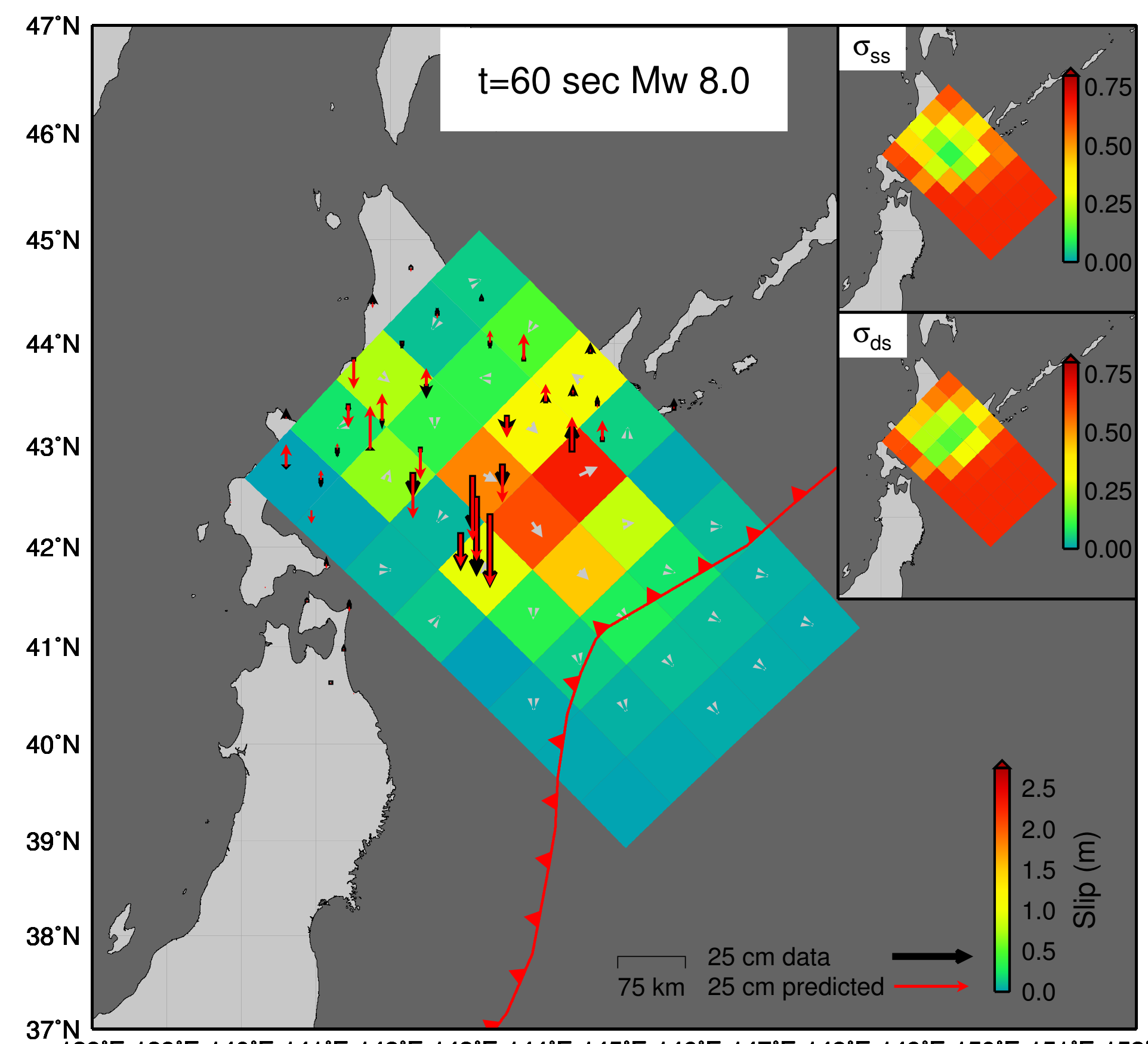

$138^{\circ} \mathrm{E} 139^{\circ} \mathrm{E} 140^{\circ} \mathrm{E} 141^{\circ} \mathrm{E} 142^{\circ} \mathrm{E} 143^{\circ} \mathrm{E} 144^{\circ} \mathrm{E} 145^{\circ} \mathrm{E} 146^{\circ} \mathrm{E} 147^{\circ} \mathrm{E} 148^{\circ} \mathrm{E} 149^{\circ} \mathrm{E} 150^{\circ} \mathrm{E} 151^{\circ} \mathrm{E} 152^{\circ} \mathrm{E}$

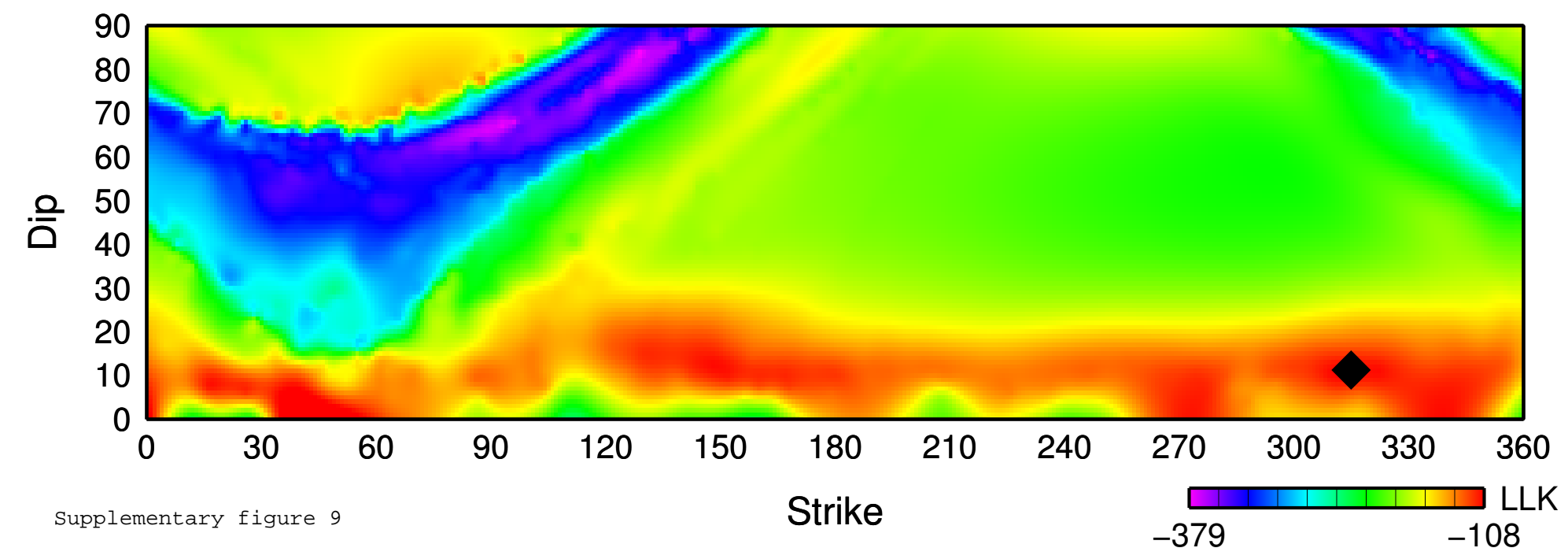




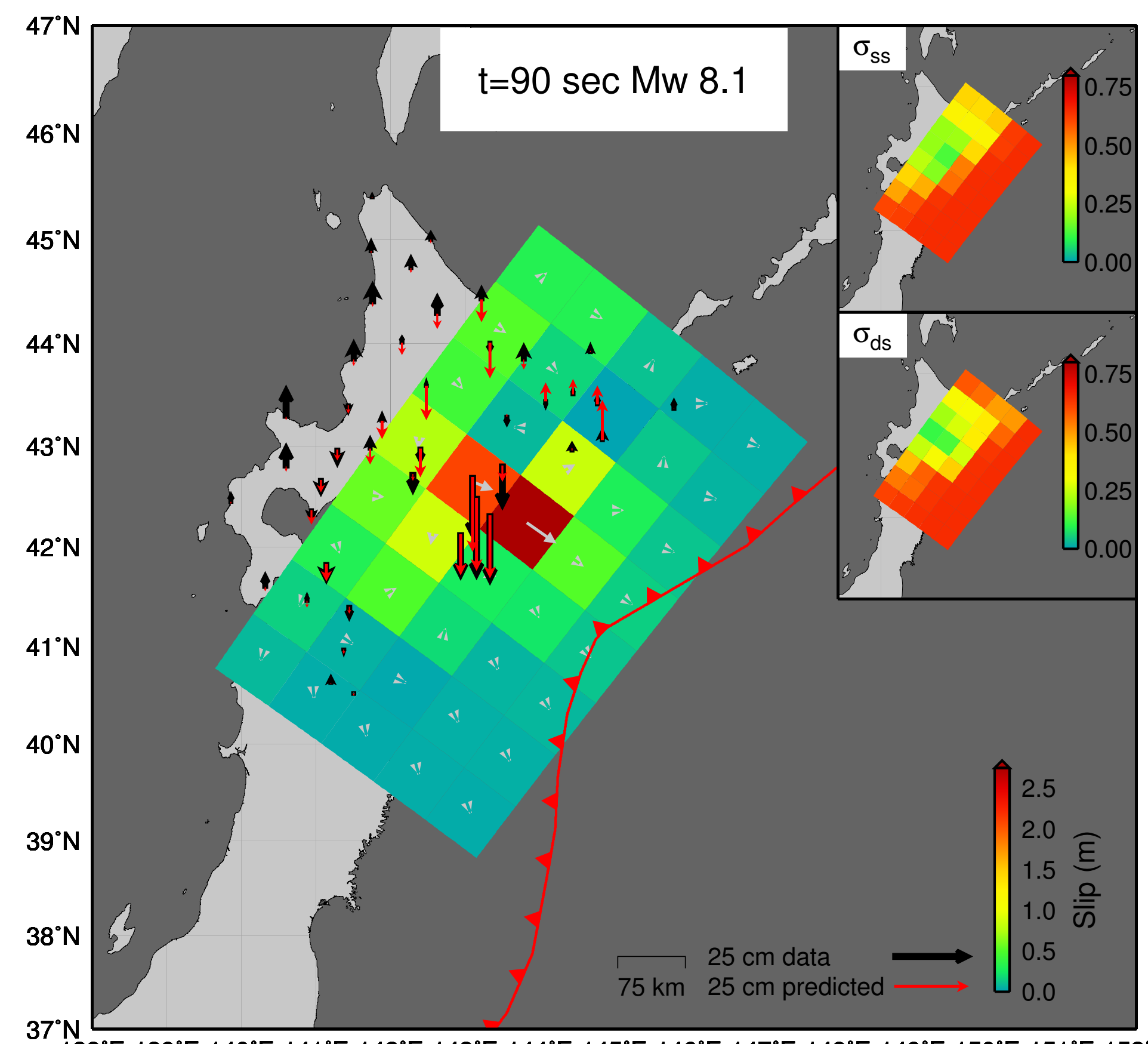

$138^{\circ} \mathrm{E} 139^{\circ} \mathrm{E} 140^{\circ} \mathrm{E} 141^{\circ} \mathrm{E} 142^{\circ} \mathrm{E} 143^{\circ} \mathrm{E} 144^{\circ} \mathrm{E} 145^{\circ} \mathrm{E} 146^{\circ} \mathrm{E} 147^{\circ} \mathrm{E} 148^{\circ} \mathrm{E} 149^{\circ} \mathrm{E} 150^{\circ} \mathrm{E} 151^{\circ} \mathrm{E} 152^{\circ} \mathrm{E}$

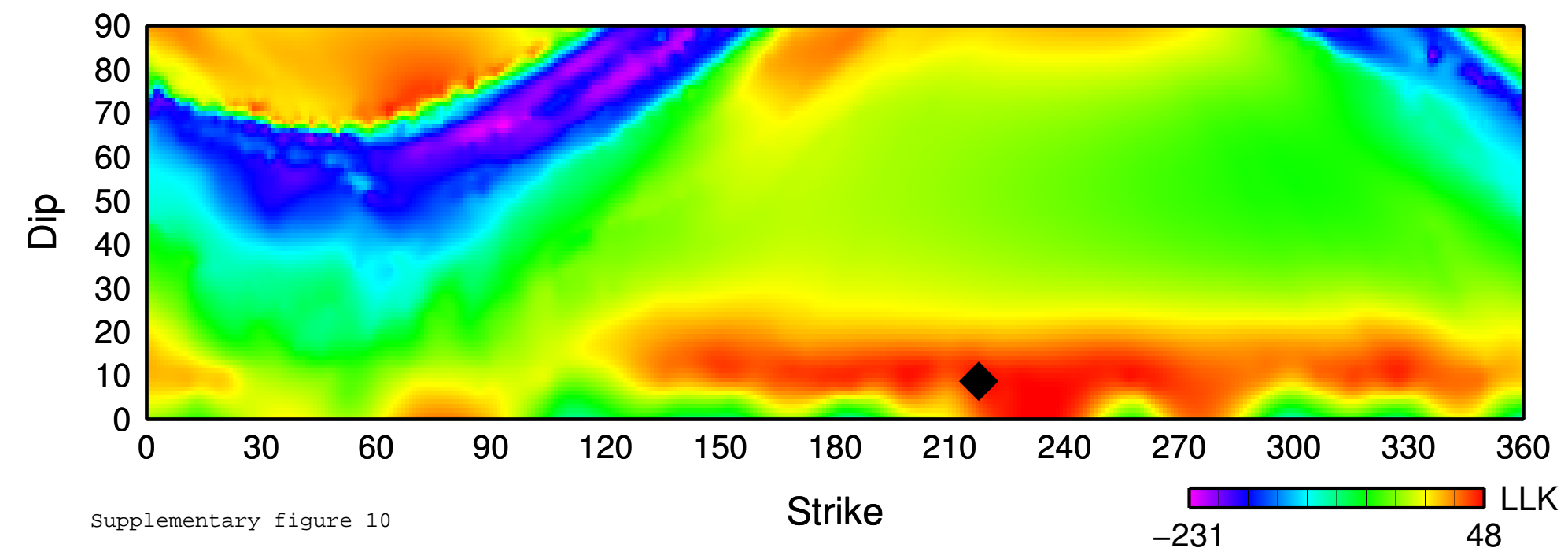



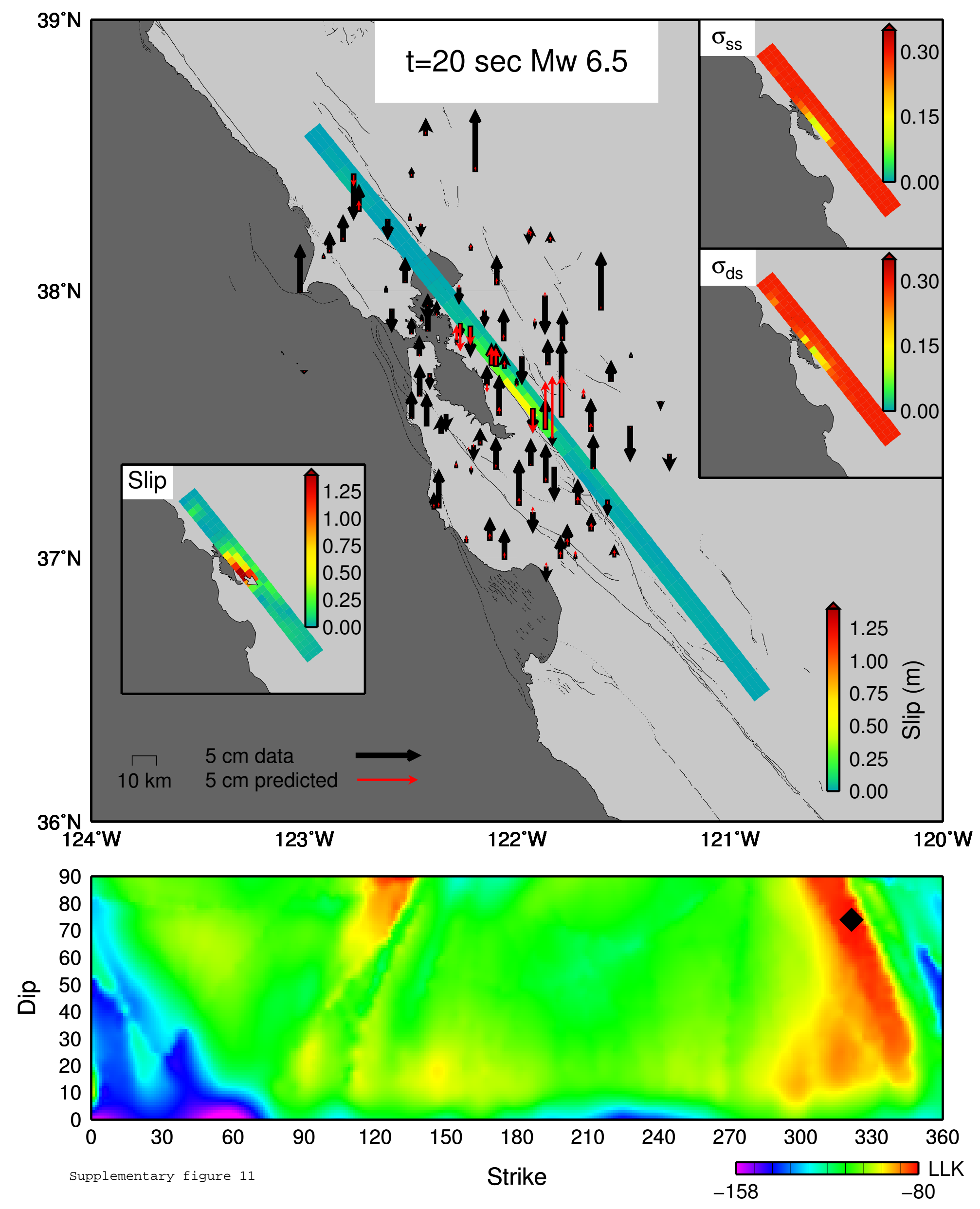

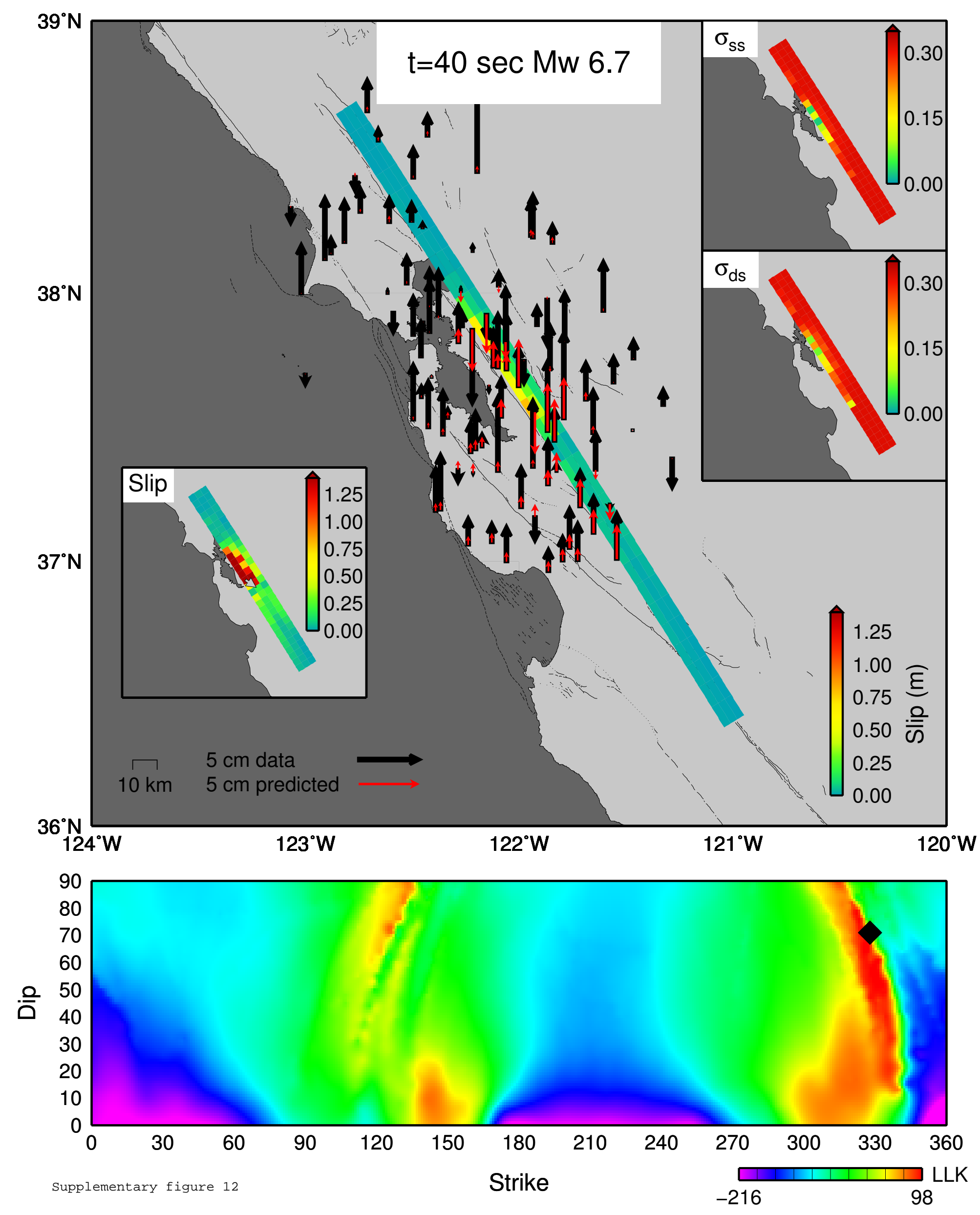


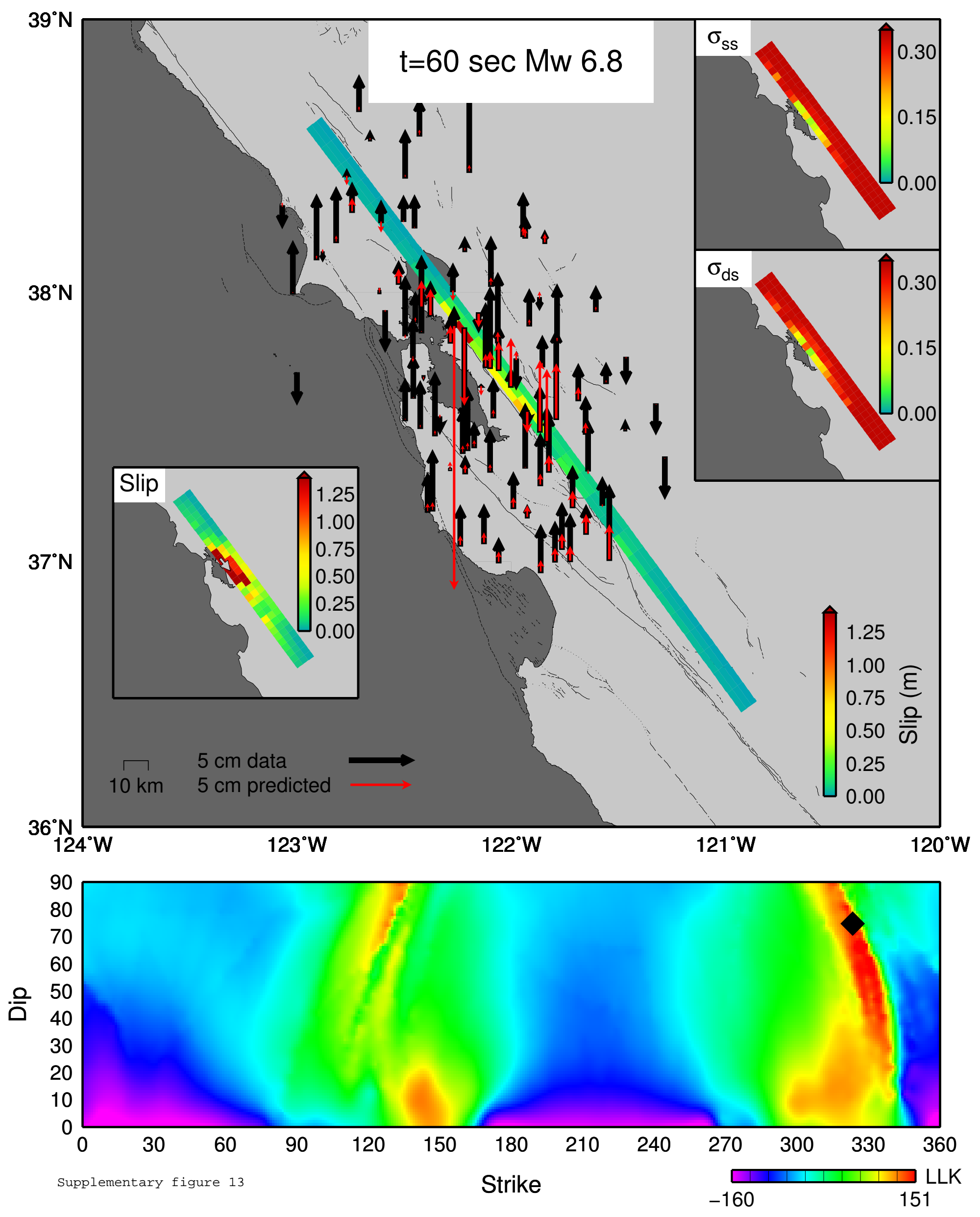




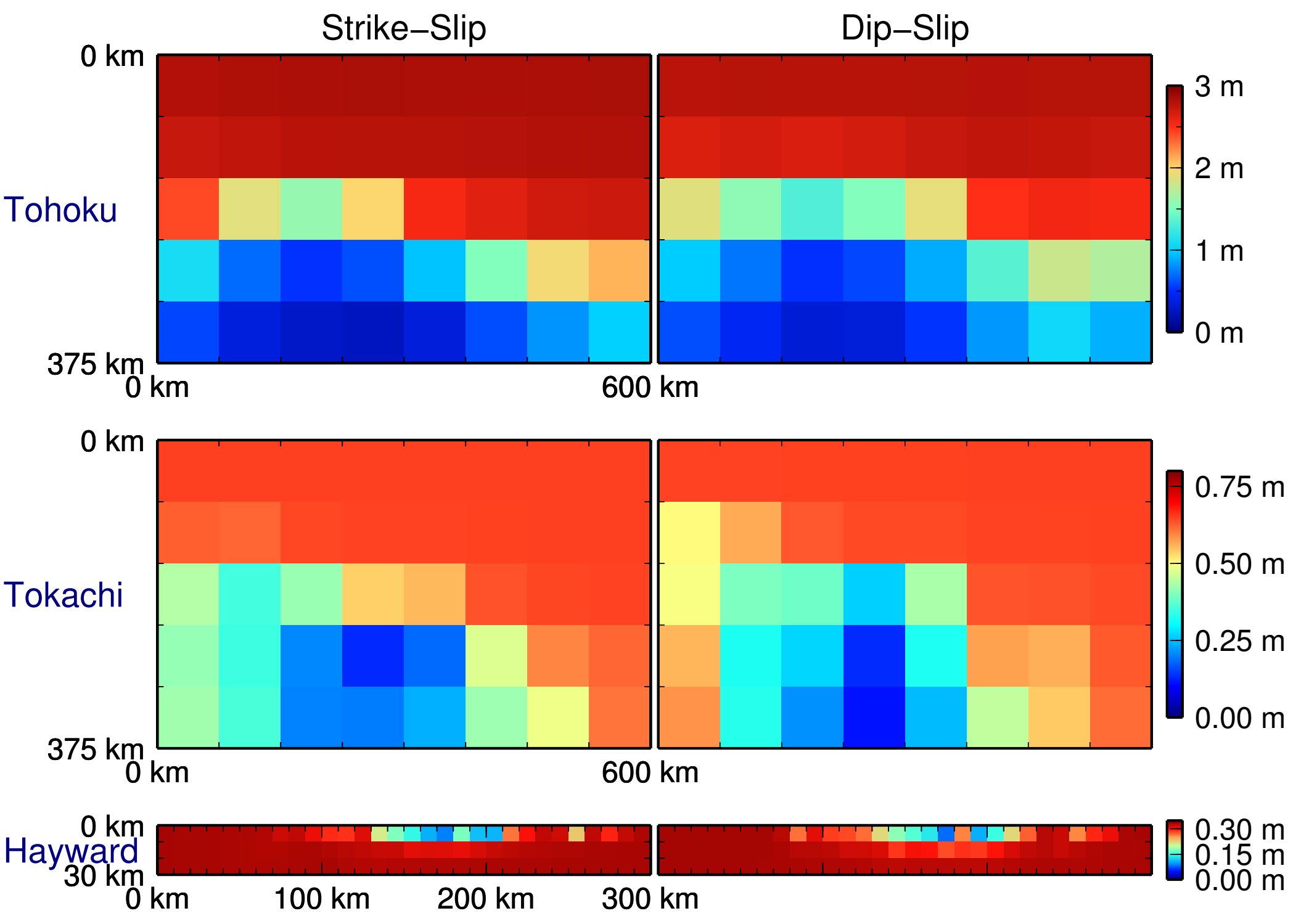




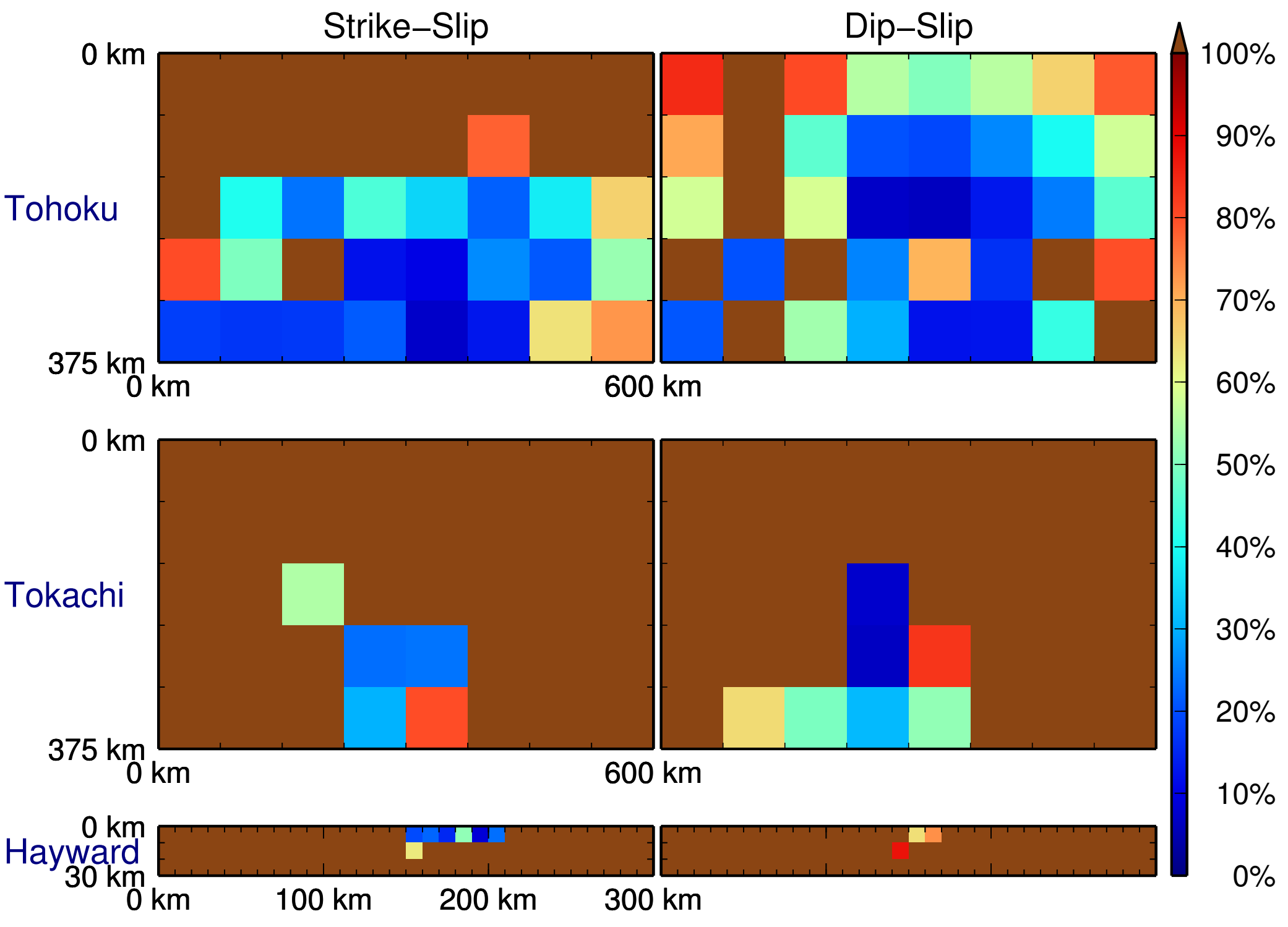

